\title{
Transcriptomic, epigenetic and metabolic characterization of the pluripotency continuum in rabbit preimplantation embryos
}

Wilhelm Bouchereau ${ }^{1}$, Luc Jouneau ${ }^{2,3}$, Catherine Archilla ${ }^{2,3}$, Irène Aksoy ${ }^{1}$, Anaïs Moulin ${ }^{1}$, Nathalie Daniel ${ }^{2,3}$, Nathalie Peynot ${ }^{2,3}$, Sophie Calderari ${ }^{2,3}$, Thierry Joly ${ }^{4,5}$, Murielle Godet ${ }^{1}$, Yan Jaszczyszyn ${ }^{6}$, Marine Pratlong ${ }^{7,8}$, Dany Severac ${ }^{7,8}$, Pierre Savatier ${ }^{1,9}$, Véronique Duranthon $^{2,3,9}$, Marielle Afanassieff ${ }^{1,9}$, Nathalie Beaujean ${ }^{1,9,10^{*}}$.

${ }^{1}$ Univ Lyon, Université Lyon 1, INSERM, Stem Cell and Brain Research Institute U1208, INRAE USC 1361, F-69500 Bron, France

${ }^{2}$ Université Paris-Saclay, UVSQ, INRAE, BREED, 78350, Jouy-en-Josas, France

${ }^{3}$ Ecole Nationale Vétérinaire d'Alfort, BREED, 94700, Maisons-Alfort, France

${ }^{4}$ ISARA-Lyon, F-69007 Lyon, France

${ }^{5}$ VetAgroSup, UPSP ICE, F-69280 Marcy l'Etoile, France

${ }^{6}$ Université Paris-Saclay, CEA, CNRS, Institute for Integrative Biology of the Cell (I2BC), 91198, Gif-sur-Yvette, France

${ }^{7}$ Univ. Montpellier, CNRS, INSERM, Montpellier France

${ }^{8}$ Montpellier GenomiX, France Génomique, Montpellier, France

${ }^{9}$ Senior authors

${ }^{10}$ Lead contact

\section{*Correspondence:}

Nathalie Beaujean (nathalie.beaujean@inserm.fr)

INSERM U1208

18 Avenue du Doyen Lépine

F-69675 Bron Cedex

France 


\section{Summary}

Despite the growing interest in the rabbit model for developmental and stem cell biology, the characterization of embryos at the molecular level is still poorly documented. We conducted a transcriptome analysis of rabbit pre-implantation embryos from E2.7 (morula stage) to E6.6 (early primitive streak stage) using bulk and single-cell RNA-sequencing, and single-cell Biomark qPCR. In parallel, we studied oxidative phosphorylation and glycolysis and analyzed active and repressive epigenetic modifications during blastocyst formation and expansion. We generated a transcriptomic, epigenetic, and metabolic map of the pluripotency continuum in rabbit preimplantation embryos and identified novel markers of naïve pluripotency that might be instrumental for deriving naïve pluripotent stem cell lines. Although the rabbit is evolutionarily closer to mice than to primates, we found that the transcriptome of rabbit epiblast cells shares common features with that of humans and non-human primates.

Keywords: Rabbit preimplantation embryo, pluripotency continuum, naïve pluripotency, embryo transcriptome, single-cell RNA-seq 


\section{Introduction}

In mammalian embryos, totipotent blastomeres become pluripotent after differentiation of the trophectoderm lineage during the morula to blastocyst transition, and form a seemingly coherent cluster of cells called the inner cell mass (ICM). After some ICM cells differentiate to the primitive endoderm (PE), pluripotency is confined to the epiblast (EPI), which will give rise to the embryo proper. Primordial germ cells (PGCs) are specified in the epiblast shortly after implantation. Ultimately, epiblast cells undergo rapid division cycles, and form an epithelialized tissue in which the posterior part gives rise to the primitive streak (PS) (Blakeley et al., 2015; Chazaud and Yamanaka, 2016; Hayashi et al., 2007; Nakamura et al., 2016; Petropoulos et al., 2016; Stirparo et al., 2018). The developmental window that extends from morula differentiation to gastrulation lasts 2-3 days in mice, compared to 7-10 days in humans and non-human primates (Nakamura et al., 2021; Shahbazi, 2020). Within that window, pluripotent cells are thought to transit through three successive pluripotency states known as the naïve, formative and primed states (Savatier et al., 2017; Smith, 2017; Takahashi et al., 2017). In mice, the ICM and epiblast cells of blastocysts, as well as the embryonic stem (ES) cell lines that are derived from them, are in the naïve state. Naïve pluripotency is associated with the expression of transcription factors Klf2, Klf4, Gbx2, Tfcp211, Esrrb, Tbx3, and Sall4 (Dunn et al., 2014), low DNA methylation (Leitch et al., 2013; Leitch et al., 2016), high mitochondrial respiration (Carbognin et al., 2016; Sone et al., 2017), and enrichment of active histone modifications at promoter regions of developmental genes (Hayashi et al., 2008). Formative pluripotency characterizes the epiblast cells of periimplantation embryos, as well as the corresponding pluripotent stem cell lines, called formative stem (FS) cells (Kinoshita et al., 2020; Smith, 2017). The formative state is associated with the expression of transcription factors Etv5, Rbpj, Tcf3, and Otx2, and intermediate DNA methylation (Kalkan et al., 2019; Kinoshita et al., 2020). Finally, the epiblast cells of early to late gastrula-stage embryos and their in vitro counterparts, epiblast stem cells (EpiSCs), are in the primed pluripotent state (Brons et al., 2007; Tesar et al., 2007). The primed state is associated with expression of Sox 3 and Oct6 transcriptional regulators (Corsinotti et al., 2017), high DNA methylation (Habibi et al., 2013; Hackett et al., 2013), low mitochondrial respiration (Zhou et al., 2012), and enrichment of repressive histone modifications at promoter regions of developmental genes (Hayashi et al., 2008; Smith, 2017). These three successive pluripotent states form the pluripotency continuum.

The characterization of the mammalian embryo transcriptome in a wide range of species is essential for unravelling the molecular mechanisms involved in pluripotency and 
understanding the adaptation of embryos in maintaining pluripotency across different developmental strategies. It is also important for the development of chemically-defined culture media, which can aid in the preservation and maintenance of naïve, formative, and primed pluripotency in embryo-derived stem cell lines. Single-cell transcriptomic data from preimplantation embryos is available in a large variety of species, including mice (Argelaguet et al., 2019; Mohammed et al., 2017; Tang et al., 2010), pigs (Kong et al., 2020; Ramos-Ibeas et al., 2019), cattle (Zhao et al., 2016), marmosets (Boroviak et al., 2018), rhesus and cynomolgus macaques (Liu et al., 2018; Nakamura et al., 2016), and humans (Blakeley et al., 2015; Petropoulos et al., 2016). Transcriptome data at pre- and mid-gastrula stages is only available in mice (Peng et al., 2016; Wen et al., 2017) and cynomolgus macaques (Nakamura et al., 2016). This later-stage transcriptome data provides the first insights into spatiotemporal alterations of gene expression occurring at gastrulation in mammals. However, it is difficult to study this developmental period, as embryos have already implanted by the gastrula stage in many species.

Despite growing interest in lagomorphs for human disease and reproductive health modelling (Duranthon et al., 2012; Fischer et al., 2012) and inter-species chimera exploration (Aksoy et al., 2021), the transcriptome of the rabbit embryo is poorly documented (Leandri et al., 2009; Schmaltz-Panneau et al., 2014). In mice, pigs, cattle, humans, and non-human primates, gastrulation starts two to eight days after implantation, when embryos are already deeply buried in the uterine wall, making the formative- and primed-pluripotency states difficult to study (Nakamura et al., 2021; Shahbazi, 2020). In contrast, rabbit embryos do not implant until the end of day seven of development, after the onset of gastrulation (Fischer et al., 2012; Puschel et al., 2010). This strategy of lagomorph development allows easier access to a larger window of development compared to rodents and primates. For this reason, rabbit embryos are perfectly suited for an in-depth exploration of the transcriptomic, epigenetic, and metabolic changes taking place during the pluripotency continuum. In this study, we conducted a transcriptome analysis of rabbit pre-implantation embryos between E2.7 (morula stage) and E6.6 (early primitive streak stage) using three complementary techniques: bulk RNA-sequencing (Illumina NextSeq), single-cell RNA-seq (10X Genomics), and single-cell qPCR (Biomark) to study specific gene expression. In addition, we studied oxidative phosphorylation and glycolysis, and we analyzed active and repressive epigenetic modifications. With this unique combination of tools and cellular processes, we explored in detail the major alterations that occur in pluripotent cells along the pluripotency continuum of rabbit embryos. 


\section{Results}

\section{Rabbit preimplantation embryos show both similarities and differences with mice and macaques in lineage marker expression}

To obtain a transcriptional map of the rabbit preimplantation embryo development, we first performed bulk RNA sequencing of micro-dissected embryos at: E2.7 (morula), E3.5 and E4.0 (early and mid-blastocyst, respectively: inner cell mass [ICM] and trophectoderm [TE]), E6.0 (expanded blastocyst: EPI, TE and primitive endoderm [PE]), E6.3 (expanded blastocyst: anterior EPI [EPI_ant]), and E6.6 (early primitive streak-stage: EPI_ant, and TE) (Fig. 1A, S1A). Thus, a total of 51 samples (three or six biological replicates, each consisting of one to 20 embryos per stage) were paired-end sequenced on Illumina NextSeq500 instrument. Between 52 and 137 million reads were mapped to the rabbit genome (Oryctolagus cuniculus 2.0), and a mean of 56.4 to $64.7 \%$ of the pair fragments were assigned to a gene. These 51 samples separated according to developmental time and lineage when projected onto the first two components of principal component analysis (PCA) (Fig. 1B). We then investigated the expression of some known lineage markers of mouse, human, and cynomolgus macaque embryos, and represented the results on a heatmap (Fig. 1C). The TE samples (E3.5 to E6.6) were enriched in CDX2, GATA2, GATA3, KRT8, KRT18, CLDN4, FABP3 and TFAP2C transcripts. The ICM samples (E3.5 and E4.0) were enriched in transcripts of pluripotency markers FGF4, DPPA5, GDF3, SOX15, KLF4, STAT3, KLF17, ESRRB, NODAL, ZSCAN10, POU5F1 (OCT4), SOX2, NANOG, ZIC2, and ZIC3. The EPI samples (E6.0, E6.3, and E6.6, thereafter called EPI_late) differed from the ICM samples in that EPI samples had lower expression of pluripotency markers FGF4, DPPA5, GDF3, SOX15, KLF4, STAT3, KLF17, and ESRRB, and higher expression of pluripotency markers SFRP2, SEMA6A, JAKMIP2, ETV5, ZIC2, ZIC3, FGF2, FGF5, OTX2, and SOX4. Finally, the PE samples (E6.0, thereafter called PE_late) were enriched in PDGFRA, SOX17, GATA4, GATA6, HFN1B, LAMA1, FOXA2, COL4A1, RSPO3, SOX7, and TFEC transcripts. Both TE markers (GATA3 and TFAP2C) and ICM markers (DDPA5, SOX15, KLF4, STAT3, KLF17, ESRRB, ZSCAN10, POU5F1, SOX2, and NANOG) were enriched in the E2.7 morula samples, suggesting early commitment of blastomeres either to ICM or TE cells. PE markers including PDGFRA, SOX17, GATA4, GATA6, HNF1B, FOXA2, RSPO3, and SOX7 were detected in the E3.5 and E4.0 ICM samples suggesting that the PE differentiation begins as early at E3.5. Note that neither the TE markers PLAC8 or SLC7A4 expressed in humans (Blakeley et al., 
2015) nor the TE marker EOMES expressed in mice (Strumpf et al., 2005) were uniquely expressed in rabbit TE. The primate-specific EPI markers ZIC3 and FGF2 (Nakamura et al., 2016), as well as the mouse-specific EPI markers FGF5 and SOX4 (Mohammed et al., 2017), were all expressed in rabbit EPI_late. Overall, these observations suggest that rabbit gene expression patterns share characteristics with both rodents and primates, though there are some notable differences.

To describe the transcriptional map of the rabbit preimplantation embryo development more precisely, we used 10X Genomics technology to sequence the transcriptome of single cells isolated from embryos ranging from morula-stage (embryonic day E3.0) up to early primitive streak-stage prior to implantation (E6.6) (Fig. 1A). The single-cell RNA-seq analysis included additional E5.0 embryos that were not included in the bulk RNA-seq study. After quality control, we retained 17,025 high-quality single-cell transcriptomes from 529 embryos, with an average of 979 expressed genes. A total of 28 to 166 embryos and 1,170 to 4,735 cells were analyzed per developmental stage (Fig. S1B-D). In order to explore the single-cell RNAseq data in an unbiased manner, we used uniform manifold approximation and projection (UMAP) dimension reduction using the 3,000 most variable genes across all cells, accounting for the mean-variance relationship present in single-cell RNA-seq gene expression data. We found that cells separated according to developmental stage, as cells were ordered in agreement with embryonic day when projected onto the first two components (Fig. 1D). The six embryonic day clusters separated further into smaller clusters as follows: E3.0 cells (morula stage embryos) formed a cluster enriched in the pluripotency markers POU5F1 and NANOG and in the TE markers GATA2, GATA3, and TFAP2C (Figs. 1D, S1EG). Cells harvested from the E3.5 and E4.0 early blastocysts formed three distinct clusters. The first one was enriched in POU5F1, SOX2, FGF4, GDF3, SOX15, and NANOG transcripts (presumptive ICM cluster), while the other two clusters were enriched in CDX2, GATA3, TFAP2C, KRT8, KRT18, GATA2, and FABP3 transcripts (presumptive TE clusters). The pluripotency-associated gene POU5F1 and the TE-associated genes GATA3, TFAP2C, and KRT18 were still expressed in the TE and ICM clusters, respectively, but at lower levels. This indicates that full lineage segregation is not yet complete at E4.0. At E5.0, cells separate into four clusters. The first cluster was enriched in POU5F1, SOX2, GDF3, SOX15, and NANOG (presumptive EPI cluster), the second one in PDGFRA, HNF1B, LAMA1, and GATA6 (presumptive PE cluster), and the last two ones in CDX2, GATA3, TFAP2C, KRT8, KRT18, GATA2, and FABP3 (presumptive TE clusters). POU5F1 was still expressed in PE and TE, which again suggests that lineage segregation is not yet complete at E5.0. In contrast, at day 
6.0, the three lineages are separated from each other, evidenced by non-overlapping expression of POU5F1 and SOX2 (presumptive EPI cluster), PDGFRA and HNF1B (presumptive PE cluster), and TFAP2C and GATA3 (presumptive TE cluster). Based on these observations, we were able to delineate with certainty the morula, ICM, EPI, TE and PE clusters on the UMAPs.

To have a more global view of differential gene expression between the main cell clusters, a heatmap was generated using the above-mentioned markers as landmarks, with the 20 most overexpressed genes in TE vs. all, ICM vs. all, EPI vs. all, and PE vs. all (Fig. 1E). Four main gene clusters were observed, characterizing rabbit TE, ICM, EPI, and PE, respectively. In addition to the landmark markers, $F A B P 3$ and $A Q P 3$ transcripts were enriched in TE cells, whereas BAG3, KHDC3L, OOEP, JAG1 and FOLR1 were enriched in ICM cells, DNMT3B was enriched in EPI cells, and APOA1, LAMA1, and LAMB1 was enriched in PE cells. By comparison, most of the genes represented on the heatmap had similar expression profiles to those described in mouse, cynomolgus macaque, and human (Guo et al., 2010; Mohammed et al., 2017; Nakamura et al., 2016; Stirparo et al., 2018). However, two genes showed unexpected expression profile. Namely, SUSD2, a marker of ICM in primates (Bredenkamp et al., 2019) is highly expressed in rabbit TE, and CCND2, a marker of the primitive streak in mice (Wianny et al., 1998) is expressed in early and late EPI in rabbits. Taken together, these results indicate that rabbit preimplantation embryos typically express the same lineage markers as mouse and cynomolgus macaque embryos, but that there are also noticeable differences.

\section{EPI, PE, and TE lineages are established after E5.0 in rabbit embryos}

To obtain a dynamic view of lineage specification, we performed pseudo-time analyses of the single-cell RNAseq dataset based on the 1,000 most variable genes. In the first analysis, the PE cells were removed from the dataset and a pseudo-time analysis was performed with the morula, ICM and TE cells (Fig. 2A). The morula cells (E3.0) split into two main branches at E3.5, leading to either ICM or TE cells (Fig. 2B,C). At E4.0, they formed two distinct populations. A heatmap representation of the 97 most differentially expressed genes in these samples showed the following: two up-regulated genes in morula $v s$. ICM and TE (FXYD4 and S100A11); 26 up-regulated genes in ICM vs. TE lineage including GDF3, DPPA5, and POU5F1; 69 up-regulated genes in TE vs. ICM lineage, including ATP2B1, ANXA6, GATA2, OCLN, ANXA2, KRT8, KRT19, FABP3, and KRT18 (Fig. 2D). Immunostaining of rabbit embryos confirmed ICM/EPI-specific expression of SOX2 at E3.5 
and E4.0, and TE-enriched expression of TFAP2C at E4.0 (Fig. 2E). Finally, the KEGG pathway analysis of the 500 top up and down regulated genes in ICM compared to TE showed enrichment of terms involved in "DNA replication," "cell-cycle," and "mismatch repair," whereas terms involved in "oxydative phosphorylation" and disease mechanisms were downregulated (Fig. 2F). Note that two other cell populations were observed at E3.5 and E4.0 termed "TE-like" cells (Fig. 2A,C). As shown on the heatmap representation of the 100 most differentially expressed genes between TE and TE-like cells, the TE-like cells showed a significant decrease in transcripts encoding ATPase and cytochrome C oxidase, suggesting reduced mitochondrial activity (Fig. S2A). Expression of amino-acid transporters and aquaporin was also decreased in the TE-like cells compared to TE cells. Moreover, there was no clear link between TE $v s$ TE-like cells and the expression of mural $v s$ polar trophectoderm markers in humans and mice (Fig. S2B,C). Thus, the nature of TE-like cells remains unclear, and more exploration is needed to understand these two separate populations.

In the second pseudo-time analysis, all TE cells were removed from the single-cell RNAseq dataset and analysis was performed with morula, ICM, EPI and PE cells (Fig. 2G). This analysis first revealed the progressive development of morula cells to ICM (E3.0 and E3.5). From E4.0 onwards, there was a split of ICM cells into two lineages, either the EPI or the PE (Fig. 2H,I). At E5.0, EPI and PE cells formed two distinct clusters at the tip of their respective branches. A heatmap representation of the 89 most differentially expressed genes showed the following: 10 up-regulated genes in morula vs. EPI and PE, including GATA3, DPPA5, and POU5F1; 35 up-regulated genes in EPI vs PE lineage, including PRDM14, TCF7L1, GDF3, and RIF1; and 44 up-regulated genes in PE vs EPI lineage, including DKK1, LAMA1, LAMB1, RSPO3, DUSP4, and PDGFRA (Fig. 2J). Immunostaining of rabbit embryos confirmed PE-enriched expression of GATA6 and EPI-specific expression of SOX2, OCT4 and ESSRB at E4.0 and E5.0 (Fig. 2K, S2D). Immunostaining also revealed expression of the pluripotency marker DPPA5 in both PE and EPI at E4.0 (Fig. S4A). KEGG pathway analysis of the 500 most differentially expressed genes between EPI and PE showed high enrichment in the EPI for terms associated with "cell cycle", whereas the PE was notably enriched in terms associated with the "MAPK signaling pathway" (Fig. 2L). Together, these results confirm the establishment of the three main lineages, EPI, PE, and TE after E5.0 in rabbit embryos.

Specification of anterior and posterior EPI, visceral and parietal endoderm, and PGCs take place after E6.0 
EPI cells from E5.0 and E6.0/E6.6 form five clusters in the UMAP, termed EPI 5.0 (from E5.0 embryos), and EPIa, EPIb, EPIc, and EPId (from E6.0/E6.6 embryos) (Fig. 3A). A pseudo-time analysis of the EPI cells $(n=2878)$ based on the 1000 most variably expressed genes showed a split into two branches, with some EPI E5.0 cells heading towards EPIa and EPIb, and the rest heading toward EPIc and EPId (Fig. 3B,C). Although their respective positions on the UMAP might suggest EPIa and EPIb are intermediate states between EPI E5.0 and EPIc and EPId, this is not likely because EPI 5.0 cells branch out to both groups separately. To clarify the identity of these five clusters, we compared the expression of the 25 most overexpressed genes in each EPI group against the other four groups (Fig. 3D). EPIa, EPIb, EPIc, and EPId clusters showed a significant decrease in transcript levels of naïve pluripotency markers compared to the EPI 5.0 cluster, including OOEP, DPPA5, KHDC $3 L$, and PRDM14. This indicates a possible transition from naïve pluripotency to primed pluripotency at E6.0 in rabbit embryos as discussed below. EPIc showed higher transcript levels for anterior epiblast markers, including SLC7A3 and OTX2 (Peng et al., 2016), whereas EPId showed higher transcript levels for posterior epiblast and primitive streak markers, including MIXL1, EOMES, EVX1, DKK1, INKA1, FST, WNT5A, TBXT, and PITX2 (Fig. S3A,B). This indicates that EPIc and EPId correspond to anterior EPI (EPI_ant) and posterior EPI (EPI_post), respectively. Surprisingly, all pluripotency and early lineage marker genes examined were downregulated in the EPIa and EPIb clusters compared to the other groups (Fig. S3A). The identity of the corresponding cells remains uncertain. Consequently, these cells were removed from all subsequent analyses.

To characterize mesodermal vs. endodermal segregation, we examined the EPI_post cells from E6.6 embryos $(\mathrm{n}=237)$. We performed a PCA using five anterior epiblast markers (SLC7A3, SLC2A1, ENPP2, ITGA6, NAV2), five early endodermal markers (CHRD, CER1, GSC, OTX2, HHEX), and five early mesodermal markers (TBXT, HAND1, LEF1, PDGFRA, WNT5A). Cells formed two separate clusters in a PCA, with one cluster having higher expression of mesodermal markers, including TBXT, HAND1, LEF1, PDGFRA, and WNT5A, and the other cluster having higher expression of definitive endoderm markers, including CHRD, CER1, GSC, OTX2, and HHEX (Fig. S3C). TBXT expression was validated by immunostaining in E6.0 and E6.6 embryos (Fig. 3E-H). These results are also in line with a previous study by Viebahn and colleagues, who reported the appearance of TBXT (Brachyury)-expressing cells between E6.25 and E6.5 in the posterior epiblast of rabbit embryos (Hassoun et al., 2009a; Viebahn et al., 2002). 
We sought to characterize the segregation of the primitive endoderm into the visceral and parietal endoderm. In marmosets and humans, OTX2 is expressed in the primitive endoderm (Boroviak et al., 2018). However, in marmosets, its expression is suppressed in the parietal endoderm and is only maintained in the visceral endoderm. E4.0 and E5.0 rabbit embryos exhibited OTX2 expression in endoderm cells (Fig. S3D). OTX2 expression then became restricted to a sub-cluster of endoderm cells at E6.0 and E6.6. To characterize these OTX2-positive cells, we performed immunofluorescence analysis of E6.0 embryos. Strong OTX2 expression was observed in SOX2-negative visceral endoderm cells underlying the layer of SOX2/OTX2 double-positive EPI cells (Fig. S3E). These results suggest that segregation of visceral and parietal endoderm takes place at E6.0 in rabbit embryos, before embryo implantation.

Finally, we sought to identify primordial germ cells (PGCs) from those expressing NANOS3, PRDM1/BLIMP1, and TFAP2C in the 10X Genomics dataset of E5.0, E6.0 and E6.6 embryos. A few cells co-expressing PRDM1/BLIMP1 and NANOS3, PRDM1/BLIMP1 and TFAP2C, or TFAP2C and NANOS3 were identified from the EPI_post (EPId) cluster (Fig. 3I). Immunofluorescence analysis showed cells positive for TFAP2C and TBXT (Fig. 3E), and SOX17 and TBXT (Fig. 3F) in the posterior epiblast at E6.6. These double positive cells were not observed in E6.0 embryos (Fig. 3G,H). These results are in line with previous observations (Hassoun et al., 2009b; Hopf et al., 2011), and confirm PGCs arise between E6.0 and E6.6 in rabbit embryos.

\section{Gradual alteration of the transcriptome in the pluripotency continuum}

To characterize the transcriptomic changes taking place in the pluripotency continuum of rabbit embryos, we examined the expression of known markers of naïve, formative and primed pluripotency. First, we analyzed 30 markers in the bulk RNAseq dataset from morula (E2.7), ICM (E3.5/E4.0), and EPI_late (E6.0/E6.3/E6.6). A heatmap representation showed a stronger expression of naïve pluripotency genes in morula and ICM, and a stronger expression of formative and primed pluripotency genes in EPI_late (Fig. 4A). Gene ontology (GO) and KEGG pathway analysis based on the 500 most differentially expressed genes between ICM and EPI_late showed enrichment for "reproduction," "embryonic organ development," "signaling pathways regulating pluripotency," and "positive regulation of cell differentiation" (E3.5/E4.0), compared to enrichment for "ion transmembrane transport," "cell adhesion molecules," and "cell-cell signaling," in EPI_late cells (E6.0/E6.3/E6.6) (Fig. S4B,C). These 
results suggest that the epiblast cells of rabbit embryos between E3.5 and E6.6 encompasses the whole pluripotency continuum.

Second, we used the 10X Genomics single-cell dataset to increase the resolution of the analysis. To focus the analysis on the pluripotent cells, cells expressing TE and PE markers, and the aforementioned EPIa and EPIb clusters, were removed. The resulting "PLURI dataset" included 4018 cells from six developmental stages (E3.0, $\mathrm{n}=875$; E3.5, $\mathrm{n}=471$; E4.0, $\mathrm{n}=580 ; \mathrm{E} 5.0, \mathrm{n}=486 ; \mathrm{E} 6.0, \mathrm{n}=937 ; \mathrm{E} 6.6, \mathrm{n}=669)$. A heatmap was generated grouping 20 landmark genes from the literature, with the 20 most overexpressed genes in ICM vs. all, EPI-5.0 vs. all, EPI_ant vs. all, and EPI_post vs. all (Fig. 4B,D). Both morula and ICM cells (E3.5/E4.0) had a higher expression of naïve pluripotency markers ESSRB, ZFP42 (REX1), DPPA5, GDF3, Tudor and $\mathrm{KH}$ domain-containing protein-encoding TDRKH, aquaporin-encoding $A Q P 3$, RNA-binding protein-encoding $O O E P$, chaperone regulatorencoding BAG3, ATP-dependent helicase-encoding $D D X 43$, unfolded protein responseencoding HERPUD1, and folate receptor-encoding FOLR1. These markers declined in EPI 5.0 , concomitantly with the upregulation of a new set of markers, including formative pluripotency markers TCF7L1, ETV4, and ETV5. Other markers enriched in EPI E5.0 included claudin-encoding gene CLND10, creatine kinase B $(C K B)$, ornithine aminotransferase-encoding gene $O A T$, aldehyde dehydrogenase-encoding gene ALDH7Al, annexin-encoding gene $A N X A 2$, calcium-binding protein-encoding gene S100A6, and acyltransferase-encoding gene GPAT3. An increase in the transcription of these genes might reflect an alteration in metabolic pathways and cell-cell interactions taking place during a change from the naïve to formative pluripotency state. Finally, in parallel with the formative/primed pluripotency markers TCF7L1, ETV5, and OTX2, a set of genes was upregulated in both EPI_ant and EPI_post in E6.0/E6.6 embryos. They included metallothionein-encoding genes $M T 1 A, M T 2 A$ and $M T 2 D$, fatty acid-binding proteinencoding genes FABP5 and FABP7, lactate dehydrogenase-encoding gene $L D H A$, and pyruvate kinase-encoding gene PKM (Fig. 4B,D). This suggests alterations in metabolic pathways concomitantly with the transition from the formative to primed pluripotency state. Secreted frizzled-related protein-encoding gene $S F R P 2$, insulin-like growth factor binding protein-encoding gene $I G F B P 2$, and cell-cycle regulator $C C N D 2$ were also up-regulated during this transition (Fig. 4B,D). EPI_post cells expressed higher levels of MIXL1, WNT5A, EOMES, FGF19, and DKK1, indicating commitment to primitive streak and mesoderm. Differential expression of ESRRB, OTX2, FOLR1, and CD57 was confirmed by immunofluorescence (Fig. 4C); ESRRB and FOLR1 were more strongly expressed in the 
ICM of E4.0 embryos compared to EPI of E6.0 embryos, whereas OTX2 and CD57 were more strongly expressed in the EPI_late than in the ICM. GO and KEGG pathway enrichment analysis based on the 500 most differentially expressed genes between early (ICM E3.5/E4.0) and late (EPI_ant/EPI_post E6.0/E6.6) epiblast cells showed enrichment for "biosynthetic, metabolic and transport processes" in ICM/EPI cells, and enrichment for "cell death" and "Wnt signaling pathway" in EPI_ant/EPI_post cells (Fig. S4D,E).

To further characterize the expression pattern of some key pluripotency genes at the single-cell level, single-cell Biomark qPCR was used to analyze morula, ICM and epiblast cells collected from E3.0, E3.5, E4.0, E5.0, E6.0 and E6.6 embryos (Figs. 4E, S4F). 260 cells were analyzed for each of the six developmental stages. Naïve-pluripotency markers $D A Z L$, ESRRG, KLF5, DPPA2, OOEP, FGF4, DPPA3, and ZFP42 were expressed at relatively higher levels in morula (E3.0), ICM (E3.5/E4.0) and EPI (E.5.0) cells compared to cells from later embryo stages. In contrast, the primed pluripotency markers $C D H 3$ and $C E R I$ were expressed at relatively lower levels in morula (E3.0), ICM (E3.5/E4.0) and EPI (E.5.0) compared to cells from E6.0 and E6.6 embryos. Interestingly, FBXO15 and TDGF1/CRIPTO, markers of naïve pluripotency in mice, were upregulated in E6.0 and E6.6 EPI cells from rabbit embryos. Taken together, the results of the 10X Genomics and Biomark qPCR analyses allowed us to identify gene markers of the naïve, formative and primed pluripotency states in rabbit preimplantation embryos, including genes involved in metabolic pathways, cell-cell interactions, and signaling pathways. We also identified notable differences with mouse and cynomolgus macaque embryos.

The "PLURI dataset" was further used to investigate the expression of cell-cycle genes. The Seurat's cell-cycle scoring and regression algorithm was used to determine the proportion of cells in each phase of the cell cycle (Scialdone et al., 2015). An overall decrease of the proportion of cells in G1 phase was observed in ICM and EPI cells during morula to blastocyst transition and blastocyst maturation (Fig. S4G). This decrease was associated with concomitant alterations of cell cycle regulatory gene expression including cyclin-, cyclindependent kinase- (CDK), and CDK inhibitor-encoding genes (bulk RNA-seq dataset; Fig. S4H). To identify key changes, we analyzed the "PLURI dataset" for expression of cell-cycle regulators (Fig. S4I). Increased expression of positive regulators of the G1/S transition including cyclin D2 (CCND2) and cyclin E (CCNE1) was observed at E5.0 and E6.0, a finding consistent with the shortening of the G1 phase in late epiblast cells (Fig. S4G). Increased expression of $C U L 3$ and $F B X W 7$ was also observed during the transition from early to late epiblasts. $C U L 3$ and $F B X W 7$ encode components of the E3 ubiquitin ligase complex 
that targets cyclin E for degradation after S-phase entry. Their upregulation might reveal the onset of oscillatory expression of cyclin E and cyclin E-CDK2 complexes in the cell cycle. Increased expression of ataxia telangiectasia mutated (ATM) and rad3-related (ATR), checkpoint kinase 2 (CHEK2) and TP53 was also observed during the transition from early to late epiblasts, which might reveal the activation of ATM-ATR/CHEK/p53 pathway for DNA damage repair at E5.0/E6.0 as previously suggested (Osteil et al., 2016). Overall, these results strongly suggest that changes in cell cycle regulation take place in the pluripotency continuum of rabbit embryos.

\section{Modification of the epigenetic landscape in the pluripotency continuum}

We investigated the DNA methylation dynamics during rabbit preimplantation development using immunodetection of 5-methylcytosine (5meC) and 5-hydroxymethylcytosine (5hmeC) in E3.0 to E6.6 embryos (Fig. 5A). 5meC fluorescence was low in morulae (E3.0) and early blastocysts (E3.5) compared to later stages. There was also significantly higher 5meC fluorescence in EPI cells compared to TE cells in E6.0 and E6.6 embryos. The dynamics of $5 \mathrm{hmeC}$ immunostaining appeared to correlate inversely with that of 5meC. Morulae (E3.0), ICM (E3.5), and TE (E3.5) all had higher levels of 5hmeC fluorescence compared to later stages embryos, indicating active demethylation at the early stages. Whereas $5 \mathrm{hmC}$ signal was almost absent in all tissues at E5.0, it could again be detected at E6.0 and E6.6 in EPI cells. The 5meC and 5hmeC fluorescence patterns are consistent with the increasing expression of DNA methyltransferases (DNMTs) and the decreasing expression of ten-eleven translocation (TET) methylcytosine dioxygenases TET1 and TET2, observed during the transition from ICM to EPI (Fig. 5B,E and S5A). Note, however, that TET3 expression increased between the ICM and late EPI stages, which may explain why $5 \mathrm{hmeC}$ is still detected in the EPI at E6.0 and E6.6, despite the increased level of $5 \mathrm{meC}$ and increased expression of DNMTs (Fig. 5A,E and S5A). Thus, the rabbit embryo shows DNA methylation dynamics similar to those previously described in mice and humans (Zhang et al., 2018; Zhu et al., 2018).

Through the study of $\mathrm{X}$ chromosome coating by XIST RNA, it was shown that $\mathrm{X}$ inactivation begins at the morula stage in the rabbit (Okamoto et al., 2011). To describe this process in more detail across the pluripotency continuum, we analyzed H2AK119ub and H3K27me3 marks, two post-translational modifications of histones associated with $\mathrm{X}$ chromosome inactivation in mice (Chaumeil et al., 2011). At the morula stage, H2AK119ub immunostaining appears as small diffuse nuclear spots (E3.0) (Fig. 5C). From the early 
blastocyst stage onward, labeling begins to form foci in half of the embryos analyzed $(\mathrm{n}=$ 34). In those, the percentage of cells with a single nuclear focus increased from $15 \%$ (E3.5) to $100 \%$ (E5.0). Immunostaining of the repressive mark H3K27me3 revealed a similar dynamic, starting with $18 \%$ of the early blastocysts (E3.5) having a large H3K27me3 nuclear foci ( $\mathrm{n}=$ 11, Fig. S5B), and plateauing at 50\% of the embryos from E5.0 onward ( $\mathrm{n}=12$, Fig. 5D). Consistent with the concomitant accumulation of both H2AK119ub and H3K27me3 marks between E3.5 and E5.0, the bulk RNAseq dataset revealed that expression of the H2AK119ub erasers, ASXL2 and ASXL3, as well as expression of the H3K27me3 erasers, KDM6A and $K D M 6 B$, were downregulated during the transition from ICM to EPI_late (Fig. 5E).

To directly investigate the transcriptional activity of the two $\mathrm{X}$ chromosomes, we measured the expression of X-related genes relative to that of genes located on autosomes 2, 3, 4 and 5 in the 10X Genomics dataset. When both $\mathrm{X}$ chromosomes are expressed, the ratio between these two types of genes is equal to 1 (R1), as observed in half of the cells at E3.0 and E4.0 (Fig. 5F). When one of the X-chromosomes is transcriptionally inactive, the ratio is equal to 0.5 (R0.5). Because transcripts of the SRY gene (the only gene sequenced on the rabbit Y chromosome) were not detected in our dataset, we were unable to determine cell genders. We assumed that the R0.5 cells observed at E3.0 and E4.0 are male with a single X chromosome, while the R1 cells are female with both active $\mathrm{X}$ chromosomes. From E4.0 onwards, the R1 population gradually disappears in favor of the R0.5 population and it has completely disappeared at E6.0. These results corroborate the silencing of the early Xinactivated gene HPRT1, observed at E4.0-E5.0 in female rabbit embryos (Okamoto et al., 2011). From these results, we conclude that $X$ chromosome inactivation by repressive marks begins as early as E3.5, followed by transcriptional inactivation, and ends at E6.0.

\section{Gradual switch from OXPHOS to glycolysis-dependent metabolism in the pluripotency continuum}

The bulk RNAseq dataset was used to investigate the expression of genes associated with oxidative phosphorylation (OXPHOS) and glycolysis. A stronger expression of both nuclear (NC)- and mitochondrial (MT)-encoded genes related to OXPHOS metabolism was observed in TE and ICM cells compared to EPI_late (Fig. 6A). These include genes of the $N D U F$ family encoding subunits of the NADH-ubiquinone iron-sulfur oxidoreductase complex, the cytochrome c oxidase $(C O X)$ and ubiquinol-cytochrome c reductase (UQCR) encoding subunits of the electron transport chain, and genes encoding subunits of the $\mathrm{F}_{\mathrm{O}}$ and $\mathrm{F}_{1}$ regions of ATPase. Analysis of $N D U F V 2, U Q C R Q, C O X 8 A$, and ATP5PD in the single- 
cell "PLURI dataset" confirmed this observation, showing a gradual downregulation of gene expression between E3.0 and E6.6 (Fig. 6B). In contrast, lactate dehydrogenase-encoding genes, $L D H A$ and $L D H B$, and pyruvate kinase-encoding gene, $P K M$, which are key genes of glycolysis, were expressed at much higher levels in EPI_late compared to ICM (Fig. 6A). The single-cell "PLURI dataset" confirmed this finding, showing a gradual increase in the expression of $L D H A, L D H B$, and $P K M$ between E3.0 and E6.0 (Fig. 6B). These observations are consistent with a switch from OXPHOS to glycolysis for energy production between early blastocysts and pre-gastrula stage embryos in rabbits, which correlates with observations made in mice (Houghton, 2006). However, other genes involved in glucose uptake and metabolism, including the soluble carrier family-encoding genes $S L C$, hexokinase-encoding genes $H K$, and pyruvate dehydrogenase complex-encoding genes $P D H$, were already expressed at early embryo stages (ICM and E3.0 to E4.0), which suggests that the pluripotent cells of the ICM and early EPI are poised for upregulating glucose metabolism-based energy production.

To investigate mitochondrial activity during rabbit preimplantation embryo development, embryos between E3.0 and E6.6 were treated with tetramethylrhodamine ethyl ester (TMRE), which reveals mitochondrial membrane depolarization ( $\Delta \Psi \mathrm{m})$. Morula and TE cells showed strong TMRE labeling, indicating high $\triangle \Psi \mathrm{m}$ and strong OXPHOS activity (Fig. 6C). TMRE labeling was much lower in the ICM and EPI cells of E3.5 to E6.0 embryos, and became undetectable by E6.6. This result was confirmed by CellROX assay, which labels the reactive oxygen species (ROS) produced by OXPHOS (Fig. S5C). These results are consistent with the expression pattern of NDUF, COX, UQCR, and ATP5 families of genes, which are highly expressed in TE cells, and have low expression in EPI_late cells. Fluorometric determination of 2-deoxyglucose incorporation was performed to study glycolysis in rabbit embryos (Fig. 6C). Weak 2-deoxyglucose fluorescence was observed in morulae (E3.0), but gradually increased in later stages (from E3.5 onwards), first in TE cells and then in EPI cells. Strong fluorescence was observed in the primitive streak region in E6.6 embryos, correlating with the higher expression of $L D H A$ and $P K M$ observed in transcriptome studies (Fig. 6B). Activity of HIF-1 $\alpha$, a master regulator of the metabolic shift from OXPHOS to glycolysis, was investigated using the Image-iT Hypoxia reagent. A peak of HIF-1 $\alpha$ expression and activity was observed in E3.5-E4.0 embryos (Fig. 6D), further suggesting a switch from OXPHOS to glycolysis-dependent metabolism during the transition from early to late EPI. 


\section{Robust markers of naive pluripotency in rabbits are common to either mice or primates and rarely to both}

We sought to further characterize the naïve pluripotency state in rabbit embryos and compare to current data in mice and primates. To address this, we identified the differentially expressed genes between ICM (E3.5/E4.0) and TE (E3.5/E4.0/E6.0/E6.6), ICM (E3.5/E4.0) and PE_late (E6.0), and ICM (E3.5/E4.0) and EPI_late (E6.0/E6.3/E6.6) in the bulk RNAseq dataset, which resulted in 3,237 differentially expressed genes in ICM cells compared to the EPI, TE, and PE lineages ( $<$ 0.01) (Fig. 7A). This analysis revealed an upregulation of pluripotency markers DPPA5, KDM4A, IL6R, SOX15 and NODAL, and a downregulation of early differentiation markers (e.g. SEMA6A, CCND2, APOA1, LAMB1, CLDN4, and KRT8) in ICM cells compared to the other lineages (Fig. 7B). GO analysis highlighted the underrepresentation of metabolic and adhesion processes in ICM cells, including "Small molecule metabolic process", "Organic acid metabolic process," and "Cell-substrate adhesion" (Fig. 7C). KEGG pathway analysis showed an ICM enrichment in genes related to pluripotency, including "Signaling pathways regulating pluripotency of stem cells" and "Pathways in cancer" (Fig. 7D).

We performed a similar differential analysis between ICM cells (E3.5/E4.0) compared to other cell types (E3.5/E4.0/E5.0/E6.0/E6.6) in the 10X-genomics dataset (average log Fold change > 0.25), and identified 140 differentially expressed genes (Fig. 7E). GO analysis of these 140 genes showed enrichment for biological processes related to high transcription and cell division, including "chromatin organization," "Histone modification," "DNA repair," and "cellular response to DNA damage stimulus" terms (Fig. 7F). Among these 140 genes were pluripotency-associated genes DPPA5, GDF3, PRDM14, SOX15, chromatin regulatorencoding genes KDM4A, BRDT, KMT2C, SMARCAD1, and DNA-damage associated genes RIF1, MSH6, RRM2, and PARP1. The highest expression of KDM4A transcripts, as well as ICM-specific expression of KDM4A protein, were observed at E4.0 (Fig. 7G,H). These genes might be instrumental to characterize naïve pluripotency in embryo-derived and induced pluripotent stem cell (PSC) lines. We next applied Gene Set Enrichment Analysis (GSEA) to the 10X-genomics dataset, in which the aforementioned 140 genes occupied the highest ranks from all differentially-expressed genes. A Cytoscape representation revealed an enrichment in genes related to "DNA replication and repair," and a depletion in genes related to "Oxphos," "Adhesion and ECM interaction," and "PPAR signaling" (Fig. 7I). We then further examined the JAK-STAT, NOTCH, WNT, MAPK, and TGF-beta signaling pathways, all of which are associated with the regulation and maintenance of pluripotency in rodents and primates 
(Boroviak et al., 2015; Boroviak et al., 2018; Mohammed et al., 2017; Nakamura et al., 2016). Consistent with naïve pluripotency data in mice (Mohammed et al., 2017), WNT, TGF-beta and MAPK signaling pathways were all downregulated in ICM cells versus TE, PE and late EPI cells (Fig. S6A). In contrast, the expression of components of the JAK-STAT signaling pathway, including IL6R, IL6ST, IL4R, STAT4 and STAT3, were increased (Fig. S6A,B). Notably, the expression of NOTCH and NOTCH target gene HES1 were low, whereas the expression of the NOTCH ligand JAG1 and the NOTCH inhibitor NUMB were high in ICM cells compared with other cells (Fig. S6A,C). Low NOTCH activity in rabbit ICM is consistent with recent data indicating that treatment of human PSCs with the NOTCH pathway inhibitors, DBZ and RIN1, enhances naïve pluripotency (Bayerl et al., 2021). Overall, these results suggest that the mechanisms controlling naïve pluripotency in rabbit preimplantation embryos utilize signaling pathways that function in mice, primates, or in both.

Finally, we asked which of the aforementioned 140 genes are also specific to the ICM and early EPI cells in both mice and cynomolgus macaques, making use of the single-cell RNAseq data generated by Argelaguet and colleagues (Argelaguet et al., 2019) and Nakamura and colleagues (Nakamura et al., 2016). In these datasets, ICM and early EPI cells were identified using landmark markers of naïve pluripotency in mice (Fgf4, Esrrb and Klf2) (Fig. S7A) and cynomolgus macaques (FGF4, KLF17, and SOX15) (Fig. S7B). The 50 most overexpressed genes between ICM and early EPI cells compared to all other cell types were identified and represented by heatmaps (Fig. S7A,B). Genes more highly expressed in naïve cells were represented by volcano plots, highlighting 120 ortholog genes previously identified in rabbits (Fig. 7J,K). Of these 120 genes, 24 were also upregulated in mice (average log fold-change > 0.25) and 15 were upregulated in cynomolgus macaques (average log foldchange > 0.25). These genes are associated with "pluripotency regulation," "chromatin organization" and "DNA metabolism and repair" as shown in an interaction map of biological processes (Fig. S7C). Remarkably, $63(\sim 52 \%)$ of those were also present in the list of 3,237 differentially expressed genes in ICM vs. EPI, TE, and PE lineages from the bulk RNAseq dataset.

Finally, a comparison of the most differentially expressed genes in the three species led to the identification of five common genes, DPPA5, OOEP, RING-Type E3 Ubiquitin Transferase-encoding gene $M K R N 1$, Lysine demethylase-encoding gene $K D M 5 B$, and heatshock protein-encoding gene HSP90AAl (Fig. 7L). Notably, these genes were also present amongst differentially expressed genes in ICM cells vs. EPI and PE cells from the bulk 
RNAseq (Table S1). In addition, DPPA5, OOEP and MKRN1 were also present in the ICM vs. TE comparison (Table S1). Taken together, these results show that rabbit preimplantation embryos share some of the marker genes for naive pluripotency with those of mice, and some with those of cynomolgus macaques. 


\section{Discussion}

Our study describes the results of the first thorough investigation of the rabbit preimplantation embryos at the single-cell level. It combines bulk and single-cell RNA sequencing, protein immunolabelling, and fluorometric quantification of mitochondrial activity and glycolysis to characterize the transcriptome, epigenome and metabolome of the pluripotent cells from the morula to early gastrula stage. Four main findings emerge from this study: (1) The three early lineages, EPI, PE and TE are fully segregated between E5.0 and E6.0. (2) ICM and early EPI cells (E3.5/E4.0) and late EPI cells (E6.0/E6.6) exhibit the cardinal features associated with naïve and primed pluripotency, respectively; E5.0 is a transitional stage in that respect. (3) Novel markers of naïve pluripotency were identified, including MKRN1 and OOEP; (4) Although the rabbit is evolutionarily closer to mouse than to primates, the transcriptome of rabbit pluripotent cells shares many common features with that of humans and non-human primates, including markers of naïve pluripotency.

In Eutherian mammals, TE formation is triggered by the asymmetric segregation of keratins and polarization of the outer cells of the morula at the onset of compaction (Lim et al., 2020 Nature; Gerri et al., 2021). In our study, the onset of KRT8/18 expression, and that of GATA2 and GATA3, indicates an early start of the TE program in some morula cells. On the other hand, CDX2 is only expressed late (E4.0) in the TE of the expanded blastocyst and OCT4, initially expressed in all cells of the early blastocyst, including the TE, becomes restricted to the EPI cells only at E5.0. These results point to the late differentiation of TE lineage. It is also from E4.0 onwards that the expression of SOX2 and GATA6, markers of EPI and PE, respectively, become mutually exclusive and the two cell types separate to form two distinct cell compartments at E5.0, consistent with a previous study (Piliszek et al., 2017). These results indicate that the late segregation of EPI, TE, and PE lineages is similar to what has been described in human and non-human primates (Meistermann et al., 2021; Nakamura et al., 2016; Stirparo et al., 2018).

Recent studies in mice, cynomolgus macaques, and pigs describe the evolution of the transcriptome and epigenome of pluripotent cells from the morula stage to gastrulation, highlighting the transition from the naïve to primed pluripotency states (Argelaguet et al., 2019; Mohammed et al., 2017; Nakamura et al., 2016; Ramos-Ibeas et al., 2019). In rodents and primates, this type of analysis is more challenging because the developmental stage at which this transition occurs corresponds roughly to the time when embryos implant in the uterus. This is particularly true of primates, where access to the newly implanted embryo (between E8 and E13) is virtually impossible. In contrast, rabbit embryos are well suited for 
investigating this transition because they have delayed embryo implantation until after the onset of gastrulation (Fischer et al., 2012; Puschel et al., 2010). We found that the epiblast of E5.0 rabbit embryos is a transition state, characterized by the downregulation of naïve pluripotency markers and the upregulation of formative and primed pluripotency markers. In addition, it is at this stage that we observed the shift to predominantly glycolic metabolism, gene dosage compensation due to X-chromosome inactivation and genome re-methylation, all of which are considered as key events of the naïve-to-primed state transition in rodents and primates (Davidson et al., 2015; Devika et al., 2019). Despite embryo staging prior to cell preparation, some overlap between naïve, formative and primed markers can be observed, which may reflect asynchrony of gene expression dynamics. This heterogeneity emphasizes the transitional-stage notion associated with E5.0 rabbit embryos.

Our study identified new marker genes for naïve pluripotency in rabbit, a species in which it has not yet been possible to derive truly naïve pluripotent stem cell lines (Osteil et al., 2016; Tapponnier et al., 2017). Five of these genes, MKRN1, KDM5B, DPPA5, OOEP, and HSP90AAl are also specifically expressed in the ICM and early EPI in mouse and cynomolgus macaque embryos. MKRN1 is a target of OCT4 (Cassar et al., 2015), and encodes an E3 Ubiquitin Transferase involved in the degradation of p53 and p21 and promotes cell-cycle progression (Lee et al., 2009). MKRN1 also induces the degradation of APC and PTEN, thus participating in the activation of the WNT and PI3K signaling pathways (Lee et al., 2018; Lee et al., 2015). It also negatively regulates glycolysis metabolism via the 5'-adenosine monophosphate-activated protein kinase (AMPK) (Han et al., 2018). Thus, MKRN1 has functional characteristics expected of a naïve pluripotency regulator. $K D M 5 B$ encodes a H3K4 demethylase. Its depletion improves iPSC reprogramming (Dabiri et al., 2019; Kidder et al., 2013), and enhances self-renewal by preventing the deactivation of core pluripotency genes and the activation of differentiation genes (Kidder et al., 2013). DPPA5 encodes an RNA binding protein that interacts with mRNAs encoding pluripotency and cellcycle regulators (Tanaka et al., 2006). Moreover, DPPA5 binds NANOG and enhances its function while also preventing its degradation (Qian et al., 2016). OOEP belongs to the same family as DPPA5 (Pierre et al., 2007). Although it is known to be expressed in the ICM cells and naive PSCs in various species, its role in the control of pluripotency remains unclear (Messmer et al., 2019; Mohammed et al., 2017; Nakamura et al., 2016; Stirparo et al., 2018). Finally, HSP90AA1, which encodes heat-shock protein HSP90 $\alpha$ may be directly involved in pluripotency regulation by potentiating the nuclear translocation of STAT3 dimers and protecting OCT4 and NANOG from proteasomal degradation (Bradley et al., 2012; 
Longshaw et al., 2009; Setati et al., 2010 ). Therefore, of the five genes identified in the interspecies comparison, four are capable of interacting directly with well-characterized pluripotency regulators.

Although many of the naïve and primed pluripotency markers identified in rabbits are common to mice and primates, our study also highlights some noticeable differences. The IL6 receptor-encoding gene IL6R is expressed in the ICM of rabbit and primate embryos, but is replaced by the LIF receptor-encoding gene LIFR in mice (Boroviak et al., 2018). KLF17 is a naïve pluripotency marker in rabbits (this study) and primates, but not in mice (Stirparo et al., 2018; Boroviak et al., 2018). TDGF1 is expressed in ICM and early EPI cells but downregulated in late EPI cells in mice (Boroviak et al., 2015; Mohammed et al., 2017), whereas it is only expressed in late EPI in rabbit and cynomolgus macaque (Nakamura et al., 2016). NODAL is expressed in rabbit (this study), human (Blakeley et al., 2015; Stirparo et al., 2018), and cynomolgus macaque (Nakamura et al., 2016) ICM and early EPI cells, but it is only expressed in late EPI cells in mice (Blakeley et al., 2015; Boroviak et al., 2015; Mohammed et al., 2017). Thus, the last three genes exhibit an opposite expression pattern in rabbits (this study) and cynomolgus macaques vs. mice. Finally, some rarer genes, including GDF3 and DPPA5 exhibit a similar expression pattern in rabbits and mice, with strong expression in ICM and early EPI cells and low expression in late EPI cells, whereas they remain strongly expressed in the epiblast of E13-E14 cynomolgus macaque embryos (Nakamura et al., 2016). These interspecies comparisons clearly suggest a greater similarity between rabbits and primates in the expression of pluripotency regulators across the pluripotency continuum. Arguably, this makes the rabbit a suitable species for studying the embryo colonization capacity of human naïve PSCs and the generation of inter-species chimeras (Aksoy et al., 2021).

\section{Materials and Methods}

\section{Production and dissection of rabbit embryos}

All procedures in rabbits were approved by the French ethics committee CELYNE (approval number APAFIS\#6438 and APAFIS \#2180-2015112615371038v2), and COMETHEA $n^{\circ} 45$, registered under $n^{\circ} 12 / 107$ and $n^{\circ} 15 / 59$. Sexually mature New Zealand white rabbits were injected with follicle-stimulating hormone and gonadotropin-releasing 
hormone, followed by artificial insemination as previously described (Teixeira et al., 2018). Embryos were flushed from explanted oviducts $65-159 \mathrm{~h}$ after insemination.

For bulk RNAseq, morulae were collected at embryonic day 2.7 (E2.7), pooled by ten and immediately dry-frozen. Blastocysts were collected at E3.5 and E4.0. Mucin coat and zona pellucida (ZP) were removed by protease digestion (Sigma P8811-100MG). Zona-free embryos were subsequently cultured in TCM199 supplemented with $10 \%$ fetal bovine serum (FBS, Sigma M4530) until they regain a normal morphological aspect. Inner Cell Masses (ICMs) were separated from the trophectoderm (TE) by moderate immune-surgery: briefly, blastocysts were incubated in anti-rabbit whole goat serum (Sigma R-5131) at $37^{\circ} \mathrm{C}$ for $90 \square \min$, washed thoroughly and then incubated with guinea pig complement serum (Sigma S-1639) for 5 min. After washing in PBS supplemented with 10\% FBS, ICMs were mechanically dissociated from the TE by gentle pipetting with a glass pipette. Samples (ICM and TE) were pooled by ten and immediately dry-frozen. At E6.0, the mucoprotein layer was removed mechanically using glass microcapillaries. Zona-free embryos were opened and flattened on a plastic dish in FHM medium to expose the embryoblast with the primitive endoderm on top. The primitive endoderm (visceral part) was first dissociated by careful scratching with a glass needle, and the epiblast was then separated from the trophectoderm with a microscalpel. Samples were dry-frozen. At E6.3 and E6.6, embryos were processed as described for E6.0 embryos. The anterior part of the epiblast was isolated from the posterior epiblast by manual micro-dissection prior to dry-freezing. For E6.3 embryos, the procedure for isolating the anterior epiblast was validated a posteriori by analyzing $T B X T$ expression by RT-qPCR.

For 10X single-cell RNA-seq and Biomark single-cell qPCR, embryos collected at E3.0, E3.5, E4.0 and E5.0 were treated with protease from Streptomyces griseus (SigmaAldrich P8811) at $37^{\circ} \mathrm{C}$ followed by mechanical dissociation with glass microcapillaries to remove the mucin coat and ZP. For embryos collected at E6.0 and E6.6, mucoprotein layers were removed mechanically with forceps. The embryonic disks were then dissected mechanically with forceps and pooled prior to single-cell dissociation. For cell singularization, E3.0, E3.5, E4.0 and E5.0 embryos were treated with 0.05-0.1\% trypsin for 5$10 \mathrm{~min}$ at $37^{\circ} \mathrm{C}$. For E6.0 and E6.6 embryos, epiblast cells were mechanically dissociated to obtain small cell clusters ( $<10$ cells), which were then treated with TryPLE for 5 min at $37^{\circ} \mathrm{C}$, and singularized by gentle mechanical dissociation. Enzymatic activities were stopped by adding 10\% fetal bovine serum (Gibco 11563397). For Biomark qPCR, single-cell were harvested using glass micro-capillaries and transferred into $200 \mu \mathrm{L}$ Eppendorf tubes 
containing $5 \mu \mathrm{L}$ buffer of CellsDirect ${ }^{\mathrm{TM}}$ One-Step qRT-PCR kit (ThermoFisher) and immediately frozen at $-80^{\circ} \mathrm{C}$. For $10 \mathrm{X}$ single-cell RNA-seq, cell suspensions were run through a $50 \mu \mathrm{m}$ filter to remove any remaining cell clumps and were loaded on a Chromium controller.

\section{RNA extraction and bulk RNA sequencing}

Total RNA was isolated from batches of embryos, ICM or TE $(n=20)$ using PicoPur Arcturus (Excilone, France) with a DNase I (Qiagen, Germany) treatment as recommended by the supplier. For E6.0, E6.3, and E6.6 stages, RNAs were extracted from single embryo samples. Three nanograms of total RNA were used for amplification using the SMART-Seq V4 Ultra Low Input RNA kit (Clontech) according to the manufacturer's recommendations (10 PCR cycles were performed). cDNA quality was assessed on an Agilent Bioanalyzer 2100, using an Agilent High Sensitivity DNA Kit. Libraries were prepared from $0.15 \mathrm{ng}$ cDNA using the Nextera XT Illumina library preparation kit. Libraries were pooled in equimolar proportions and sequenced (Paired-end 50-34 bp) on an Illumina NextSeq500 instrument, using a NextSeq 500 High Output 75 cycles kit. Demultiplexing was performed (bc12fastq2V2.2.18.12) and adapters were trimmed with Cutadapt1.15, so that only reads longer than 10 bp were kept. Number of reads ranged from 52 to 137 million. Reads were mapped to the rabbit genome (Oryctolagus cuniculus 2.0). 81.8 to $85.7 \%$ (depending on samples) of the pair fragments could be aligned; 70.3 to $78 \%$ of these fragments passed the mapping filter; 56.4 to $64.7 \%$ of them were assigned to a gene.

\section{Single-cell RNA-library construction and sequencing}

Cell suspensions were loaded on a Chromium controller (10x Genomics, Pleasanton, CA, USA) to generate single-cell Gel Beads-in-Emulsion (GEMs). Single-cell RNA-Seq libraries were prepared using Chromium Single cell 3'RNA Gel Bead and Library Kit (P/N 1000075, 1000153, 10x Genomics). GEM-RT was performed in a C1000 Touch Thermal cycler with 96-Deep Well Reaction Module (Bio-Rad; P/N 1851197): $53^{\circ} \mathrm{C}$ for $45 \mathrm{~min}, 85^{\circ} \mathrm{C}$ for $5 \mathrm{~min}$; held at $4{ }^{\circ} \mathrm{C}$. After RT, GEMs were broken and the single-strand cDNA was cleaned up with DynaBeads MyOne Silane Beads (Thermo Fisher Scientific; P/N 37002D). cDNA was amplified using the C1000 Touch Thermal cycler with 96-DeepWell Reaction Module: $98^{\circ} \mathrm{C}$ for $3 \mathrm{~min} ; 11$ cycle of $98^{\circ} \mathrm{C}$ for $15 \mathrm{~s}, 63^{\circ} \mathrm{C}$ for $20 \mathrm{~s}$, and $72^{\circ} \mathrm{C}$ for $1 \mathrm{~min} ; 72^{\circ} \mathrm{C}$ for $1 \mathrm{~min}$; held at $4^{\circ} \mathrm{C}$. Amplified cDNA product was cleaned up with the SPRIselect beads (SPRI P/N B23318). Indexed sequencing libraries were constructed with SPRIselect following these steps: (1) Fragmentation end repair and A-tailing and size selection; (2) 
adapter ligation and cleanup; (3) sample index PCR and size selection. The barcode sequencing libraries were quantified by quantitative PCR (KAPA Biosystems Library Quantification Kit for Illumina platforms P/N KK4824). Sequencing libraries were loaded at $18 \mathrm{pM}$ on an Illumina HiSeq2500 using the following read length: 28 bp Read1, 8 bp I7 Index, 91 bp Read2.

\section{Biomark qPCR analysis}

Reverse transcription and specific target pre-amplification were performed on single isolated cells described before, using the SuperScript III/RT Platinum Taq mix provided in the CellsDirect ${ }^{\mathrm{TM}}$ One-Step qRT-PCR kit (ThermoFisher), and the targeted primer pairs. The expression of TATA-box binding protein (TBP) was controlled in the pre-amplified 500 samples for each of the six analyzed embryo stages (E3.0, E3.5, E4.0, E5.0, E6.0 and E6.6) in order to test their quality and filter them. Real-time PCR (qPCR) was performed using StepOnePlus real-time PCR system and Fast SYBR® Green Master Mix (Applied Biosystems). The 1560 validated samples (260 per each embryo stage) were subsequently analyzed with Universal PCR TaqMan Master Mix (Applied Biosystems) and coupled with a DNA Binding Dye Sample Loading Reagent (Fluidigm) and Evagreen (Biotium 31000) in 96.96 Dynamic Arrays on a Biomark System. Each 96.96 array allowed the study of 48 genes in 85 unique cells, using two different primer sets for each gene. Primer sequences are shown in Table S1.

\section{Bioinformatics analysis}

For bulk RNA-seq, reads were mapped to the rabbit genome (Oryctolagus cuniculus 2.0) using the splice junction mapper TopHat (version 2.0.14) associated with the short-read aligner Bowtie2 (version 2.1.0). Finally, FeatureCounts (version 1.4.5) was then used to establish a gene count table. Hierarchical clustering was computed using hclust R package with Euclidean distance metric and Ward linkage. Principal Component analysis (PCA) was made using FactoMineR (Lê et al., 2008). Data normalization and single-gene level analysis of differentially expression genes were performed using the DESeq2 (Anders and Huber, 2010). Differences were considered significant when adjusted P values (Benjamini-Hochberg) $<0.01$, and absolute fold change $\geq 2$. Log2 transformed read counts (Rlog) (after normalization by library size) were obtained with DEseq2 package and used for heatmaps.

For single-cell RNAseq, data were analyzed using the R software (version 3.6.3) and RStudio Desktop integrated development environment (IDE) for R (version 1.4, Open Source Edition). Since the rabbit genome annotation is not complete, some genes were annotated 
manually using ENSG identity. The Ensembl release 104 (Howe et al., 2021), the National Center for Biotechnology Information (NCBI database) (Schoch et al., 2020), and the g:Profiler online software (Raudvere et al., 2019) were used to convert ENSG annotations into Gene symbols. The Seurat package (version 3.1.2) was used to filter, normalize and analyze the data as described (Stuart et al., 2019). Briefly, the data were filtered by eliminating all cells with mitochondrial genome expression above $0.7 \%$, and selecting cells in which the total number of expressed genes is between 300 and 2500. Expression levels were then log-normalized and the 3000 most variable genes were identified. Principal component analysis (PCA) and uniform manifold approximation and projection (UMAP) dimension reductions were applied to the dataset to identify cell clusters. These clusters were identified using graph-based clustering algorithm on the first 20 components and annotated according to expression of lineage-specific genes and embryonic stage. Differential expression analysis was performed and genes with a log fold-change $>0.25$ or $<-0.25$ were considered significantly differentially expressed. The same workflow was applied for published data.

Temporal trajectories were created using the following additional packages: monocle (version 2.12.0), cellranger (version 1.1.0) and viridislite (version 0.4.0). Data analysis was performed as described (Trapnell et al., 2014). Briefly, differential analysis was performed on cells isolated and annotated using Seurat package, and the top 1000 most significantly differentially expressed genes were used to order the samples in pseudo-time. Stage E3.0 was set as the starting point.

Statistical enrichment analysis of Gene Ontology (GO) and Kyoto Encyclopedia of Genes and Genomes (KEGG) terms was performed using the g:Profiler online software (Raudvere et al., 2019). Gene set enrichment analysis software (GSEA) was used to identify significantly enriched KEGG terms. Cytoscape software (Shannon et al., 2003; Smoot et al., 2011) and enrichment map plug-ins were used to build and visualize networks. The node size is scaled by the number of genes contributing to each term; edges width is proportional to the overlap between each gene set and each node is colored by their enrichment score.

For Biomark analysis, samples were analyzed using the Fluidigm ${ }^{\mathrm{R}}$ Real-Time PCR Analysis Software (version 4.1.3) and the Singular ${ }^{\mathrm{TM}}$ Analysis Toolset R package (version 3.6.2). Pluripotent and extra-embryonic cells were distinguished by the expression of OCT4, SOX2, NANOG, SOX17 and CDX2, then analyzed separately.

\section{Immunofluorescence}


Embryos were fixed in 4\% paraformaldehyde (PFA) for $20 \mathrm{~min}$ at room temperature. After three washes in phosphate-buffered saline (PBS), embryos were permeabilized in PBS$0.5 \%$ TritonX100 for $30 \mathrm{~min}$. For anti-5'hydroxymethylcytosine and 5'methylcytosine antibodies, embryos were permeabilized in PBS-0.5\% Triton for 15 min, washed in PBS for $20 \mathrm{~min}$, then incubated in $2 \mathrm{M} \mathrm{HCl}$ for $30 \mathrm{~min}$. Embryos were subsequently incubated in $100 \mathrm{mM}$ Tris- $\mathrm{HCl} \mathrm{pH} 9$ for $10 \mathrm{~min}$, washed in PBS for $15 \mathrm{~min}$, and incubated in blocking solution (PBS supplemented with 5\% bovine serum albumin) for $1 \mathrm{~h}$ at room temperature. Embryos were then incubated with primary antibodies diluted in blocking solution overnight at $4^{\circ} \mathrm{C}$ (Table S1). After two washes $(2 \times 15 \mathrm{~min})$ in PBS, embryos were incubated in secondary antibodies diluted in blocking solution at a dilution of $1: 100$ for $1 \mathrm{~h}$ at room temperature. Finally, embryos were transferred through several washes of PBS before staining DNA with 4',6-diamidino-2-phenylindole (DAPI; $0.5 \mu \mathrm{g} / \mathrm{mL}$ ) or propidium iodide (PI; $1 \mu \mathrm{g} / \mathrm{mL}$ ) for $10 \mathrm{~min}$ at room temperature. Embryos were analyzed by confocal imaging (DM 6000 CS SP5; Leica). Acquisitions were performed using an oil immersion objective (40x/1.25 0.75, PL APO HCX; Leica).

\section{Detection of ROS, glycolysis, and mitochondrial membrane potential}

After gentle removal of the mucin coat with protease from Streptomyces griseus $(5 \mathrm{mg} / \mathrm{mL})$, embryos were incubated in pre-warmed pre-equilibrated $\mathrm{RDH}$ medium containing $4 \mu \mathrm{M}$ Hoechst 33342 for DNA staining and either $5 \mu \mathrm{M}$ CellROX Deep Red reagent, $5 \mu \mathrm{M}$ Image-iT Red Hypoxia reagent or $50 \mathrm{nM}$ TMRE (all from ThermoFisher Scientific) for 30-45 $\min$ at $38^{\circ} \mathrm{C}$. For glucose uptake assays, embryos were incubated for $30 \mathrm{~min}$ in the glucose uptake mix, according to the manufacturer's instructions (Abcam ab204702). Embryos were then collected and washed twice with the analysis buffer. After staining, embryos were transferred to new drops of RDH before mounting in M2 medium (Sigma) containing 20\% Optiprep (Stem Cell Technologies) and ProLong Live antifade reagent for live cell imaging (1/100 dilution, Thermofisher). Embryos were then imaged on a Leica SP5 confocal microscope with a x25 water immersion objective.

\section{Data availability}

The RNA-Seq datasets generated in this study will be deposited in NCBI.

\section{Supplemental information}

Supplemental information includes seven supplemental figures and five tables. 
Figure S1: Raw data processing and quality controls.

Figure S2: Characterization of TE and TE-like cells.

Figure S3: Characterization of late epiblast.

Figure S4: Characterization of PE/EPI segregation and analysis of genes involved in cellcycle regulation and DNA damage response.

Figure S5: Epigenetic and metabolomic dynamics.

Figure S6: Signaling pathways enrichment in EPI cells.

Figure S7: Expression of rabbit naïve pluripotency-specific genes in mouse and monkey embryos.

Table S1: Primers, antibodies and genes annotation lists.

\section{Acknowledgments}

This work was supported by the Agence Nationale pour la Recherche (contract ANR-18CE13-023; Oryctocell), the Fondation pour la Recherche Médicale (DEQ20170336757 to P.S.), the Infrastructure Nationale en Biologie et Santé INGESTEM (ANR-11-INBS-0009), the IHU-B CESAME (ANR-10-IBHU-003), the LabEx REVIVE (ANR-10-LABX-73), the LabEx "DEVweCAN" (ANR-10-LABX-0061), the LabEx "CORTEX" (ANR-11-LABX0042), and the University of Lyon within the program "Investissements d'Avenir" (ANR-11IDEX-0007). MGX acknowledges financial support from France Génomique National infrastructure, funded as part of "Investissement d'Avenir" program managed by Agence Nationale pour la Recherche (contract ANR-10-INBS-09). W.B. is a recipient of grant from the PHASE department of INRAE. We thank Emilie Bonin, Frederic Martins and Cécile Donnadieu (Genotoul-GeT-platform, INRAE, Auzeville) for Biomark analyses. We also thank the members of UE1298 SAAJ Science de l'Animal et de l'Aliment, responsible for INRAE Jouy en Josas rabbit facility; as well as the High-throughput sequencing facility of $\mathrm{I} 2 \mathrm{BC}$ for its sequencing and bioinformatics expertise.

\section{Author contributions}

Conceptualization, N.B, P.S., M.A., and V.D. ; Methodology, N.B., M.A., W.B., M.G., and V.D.; Validation, W.B., N.B., and V.D.; Formal Analysis, W.B., L.J., C.A.; Investigation, W.B., C.A., I.A., A.M., N.D., N.P., S.C., Y.J., M.P., and D.S.; Resources, T.J.; Data Curation, W.B. and L.J.; Writing - Original Draft, P.S. and N.B.; Writing - Review and Editing, W.B., M.A., and V.D.; Visualization, W.B. and N.B; Supervision: N.B.; Project Administration, M.A., N.B, and V.D.; Funding Acquisition, P.S. and V.D. 
bioRxiv preprint doi: https://doi.org/10.1101/2021.10.06.463389; this version posted October 7, 2021. The copyright holder for this preprint (which was not certified by peer review) is the author/funder, who has granted bioRxiv a license to display the preprint in perpetuity. It is made available under aCC-BY-NC-ND 4.0 International license.

\section{Declaration of interests}

The authors declare no competing interests 


\section{Literature cited}

Aksoy, I., Rognard, C., Moulin, A., Marcy, G., Masfaraud, E., Wianny, F., Cortay, V., Bellemin-Menard, A., Doerflinger, N., Dirheimer, M., et al. (2021). Apoptosis, G1 Phase Stall, and Premature Differentiation Account for Low Chimeric Competence of Human and Rhesus Monkey Naive Pluripotent Stem Cells. Stem cell reports 16, 56-74.

Anders, S., and Huber, W. (2010). Differential expression analysis for sequence count data. Genome Biol 11, R106.

Argelaguet, R., Clark, S.J., Mohammed, H., Stapel, L.C., Krueger, C., Kapourani, C.A., ImazRosshandler, I., Lohoff, T., Xiang, Y., Hanna, C.W., et al. (2019). Multi-omics profiling of mouse gastrulation at single-cell resolution. Nature 576, 487-491.

Bayerl, J., Ayyash, M., Shani, T., Manor, Y.S., Gafni, O., Massarwa, R., Kalma, Y., AguileraCastrejon, A., Zerbib, M., Amir, H., et al. (2021). Principles of signaling pathway modulation for enhancing human naive pluripotency induction. Cell Stem Cell 28, 1-17.

Blakeley, P., Fogarty, N.M., Del Valle, I., Wamaitha, S.E., Hu, T.X., Elder, K., Snell, P., Christie, L., Robson, P., and Niakan, K.K. (2015). Defining the three cell lineages of the human blastocyst by single-cell RNA-seq. Development 142, 3151-3165.

Boroviak, T., Loos, R., Lombard, P., Okahara, J., Behr, R., Sasaki, E., Nichols, J., Smith, A., and Bertone, P. (2015). Lineage-Specific Profiling Delineates the Emergence and Progression of Naive Pluripotency in Mammalian Embryogenesis. Dev Cell 35, 366-382.

Boroviak, T., Stirparo, G.G., Dietmann, S., Hernando-Herraez, I., Mohammed, H., Reik, W., Smith, A., Sasaki, E., Nichols, J., and Bertone, P. (2018). Single cell transcriptome analysis of human, marmoset and mouse embryos reveals common and divergent features of preimplantation development. Development 145.

Bradley, E., Bieberich, E., Mivechi, N.F., Tangpisuthipongsa, D., and Wang, G. (2012). Regulation of embryonic stem cell pluripotency by heat shock protein 90. Stem Cells 30, 1624-1633.

Bredenkamp, N., Stirparo, G.G., Nichols, J., Smith, A., and Guo, G. (2019). The Cell-Surface Marker Sushi Containing Domain 2 Facilitates Establishment of Human Naive Pluripotent Stem Cells. Stem cell reports 12, 1212-1222.

Brons, I.G., Smithers, L.E., Trotter, M.W., Rugg-Gunn, P., Sun, B., Chuva de Sousa Lopes, S.M., Howlett, S.K., Clarkson, A., Ahrlund-Richter, L., Pedersen, R.A., et al. (2007). Derivation of pluripotent epiblast stem cells from mammalian embryos. Nature 448, 191-195.

Carbognin, E., Betto, R.M., Soriano, M.E., Smith, A.G., and Martello, G. (2016). Stat3 promotes mitochondrial transcription and oxidative respiration during maintenance and induction of naive pluripotency. EMBO J 35, 618-634.

Cassar, P.A., Carpenedo, R.L., Samavarchi-Tehrani, P., Olsen, J.B., Park, C.J., Chang, W.Y., Chen, Z., Choey, C., Delaney, S., Guo, H., et al. (2015). Integrative genomics positions MKRN1 as a novel ribonucleoprotein within the embryonic stem cell gene regulatory network. EMBO Rep 16, 1334-1357.

Chaumeil, J., Waters, P.D., Koina, E., Gilbert, C., Robinson, T.J., and Graves, J.A. (2011). Evolution from XIST-independent to XIST-controlled X-chromosome inactivation: epigenetic modifications in distantly related mammals. PLoS ONE 6, e19040.

Chazaud, C., and Yamanaka, Y. (2016). Lineage specification in the mouse preimplantation embryo. Development 143, 1063-1074. 
Corsinotti, A., Wong, F.C., Tatar, T., Szczerbinska, I., Halbritter, F., Colby, D., Gogolok, S., Pantier, R., Liggat, K., Mirfazeli, E.S., et al. (2017). Distinct SoxB1 networks are required for naive and primed pluripotency. eLife 6, e27746.

Dabiri, Y., Gama-Brambila, R.A., Taskova, K., Herold, K., Reuter, S., Adjaye, J., Utikal, J., Mrowka, R., Wang, J., Andrade-Navarro, M.A., et al. (2019). Imidazopyridines as Potent KDM5 Demethylase Inhibitors Promoting Reprogramming Efficiency of Human iPSCs. iScience 12, 168-181.

Davidson, K.C., Mason, E.A., and Pera, M.F. (2015). The pluripotent state in mouse and human. Development 142, 3090-3099.

Devika, A.S., Wruck, W., Adjaye, J., and Sudheer, S. (2019). The quest for pluripotency: a comparative analysis across mammalian species. Reproduction 158, R97-R111.

Dunn, S.J., Martello, G., Yordanov, B., Emmott, S., and Smith, A.G. (2014). Defining an essential transcription factor program for naive pluripotency. Science 344, 1156-1160.

Duranthon, V., Beaujean, N., Brunner, M., Odening, K.E., Santos, A.N., Kacskovics, I., Hiripi, L., Weinstein, E.J., and Bosze, Z. (2012). On the emerging role of rabbit as human disease model and the instrumental role of novel transgenic tools. Transgenic Res 21, 699713.

Fischer, B., Chavatte-Palmer, P., Viebahn, C., Navarrete Santos, A., and Duranthon, V. (2012). Rabbit as a reproductive model for human health. Reproduction 144, 1-10.

Guo, G., Huss, M., Tong, G.Q., Wang, C., Li Sun, L., Clarke, N.D., and Robson, P. (2010). Resolution of cell fate decisions revealed by single-cell gene expression analysis from zygote to blastocyst. Dev Cell 18, 675-685.

Habibi, E., Brinkman, A.B., Arand, J., Kroeze, L.I., Kerstens, H.H., Matarese, F., Lepikhov, K., Gut, M., Brun-Heath, I., Hubner, N.C., et al. (2013). Whole-genome bisulfite sequencing of two distinct interconvertible DNA methylomes of mouse embryonic stem cells. Cell Stem Cell 13, 360-369.

Hackett, J.A., Sengupta, R., Zylicz, J.J., Murakami, K., Lee, C., Down, T.A., and Surani, M.A. (2013). Germline DNA demethylation dynamics and imprint erasure through 5hydroxymethylcytosine. Science $339,448-452$.

Han, H., Chae, S., Hwang, D., and Song, J. (2018). Attenuating MKRN1 E3 ligase-mediated AMPKalpha suppression increases tolerance against metabolic stresses in mice. Cell Stress 2, 325-328.

Hassoun, R., Puschel, B., and Viebahn, C. (2009a). Sox17 Expression Patterns during Gastrulation and Early Neurulation in the Rabbit Suggest Two Sources of Endoderm Formation. Cells Tissues Organs 191, 68-83.

Hassoun, R., Schwartz, P., Feistel, K., Blum, M., and Viebahn, C. (2009b). Axial differentiation and early gastrulation stages of the pig embryo. Differentiation 78, 301-311.

Hayashi, K., de Sousa Lopes, S.M., and Surani, M.A. (2007). Germ cell specification in mice. Science 316, 394-396.

Hayashi, K., Lopes, S.M., Tang, F., and Surani, M.A. (2008). Dynamic equilibrium and heterogeneity of mouse pluripotent stem cells with distinct functional and epigenetic states. Cell Stem Cell 3, 391-401. 
Hopf, C., Viebahn, C., and Puschel, B. (2011). BMP signals and the transcriptional repressor BLIMP1 during germline segregation in the mammalian embryo. Development genes and evolution 221, 209-223.

Houghton, F.D. (2006). Energy metabolism of the inner cell mass and trophectoderm of the mouse blastocyst. Differentiation 74, 11-18.

Howe, K.L., Achuthan, P., Allen, J., Allen, J., Alvarez-Jarreta, J., Amode, M.R., Armean, I.M., Azov, A.G., Bennett, R., Bhai, J., et al. (2021). Ensembl 2021. Nucleic Acids Res 49, D884-D891.

Kalkan, T., Bornelov, S., Mulas, C., Diamanti, E., Lohoff, T., Ralser, M., Middelkamp, S., Lombard, P., Nichols, J., and Smith, A. (2019). Complementary Activity of ETV5, RBPJ, and TCF3 Drives Formative Transition from Naive Pluripotency. Cell Stem Cell 24, 785-801 e787.

Kidder, B.L., Hu, G., Yu, Z.X., Liu, C., and Zhao, K. (2013). Extended self-renewal and accelerated reprogramming in the absence of Kdm5b. Mol Cell Biol 33, 4793-4810.

Kinoshita, M., Barber, M., Mansfield, W., Cui, Y., Spindlow, D., Stirparo, G.G., Dietmann, S., Nichols, J., and Smith, A. (2020). Capture of Mouse and Human Stem Cells with Features of Formative Pluripotency. Cell Stem Cell 28, 453-471 e458.

Kong, Q., Yang, X., Zhang, H., Liu, S., Zhao, J., Zhang, J., Weng, X., Jin, J., and Liu, Z. (2020). Lineage specification and pluripotency revealed by transcriptome analysis from oocyte to blastocyst in pig. FASEB J 34, 691-705.

Lê, S., Josse, J., and Husson, F. (2008). FactoMineR: an R package for multivariate analysis. J Stat Softw 25, 1-18.

Leandri, R.D., Archilla, C., Bui, L.C., Peynot, N., Liu, Z., Cabau, C., Chastellier, A., Renard, J.P., and Duranthon, V. (2009). Revealing the dynamics of gene expression during embryonic genome activation and first differentiation in the rabbit embryo with a dedicated array screening. Physiol Genomics 36, 98-113.

Lee, E.W., Lee, M.S., Camus, S., Ghim, J., Yang, M.R., Oh, W., Ha, N.C., Lane, D.P., and Song, J. (2009). Differential regulation of p53 and p21 by MKRN1 E3 ligase controls cell cycle arrest and apoptosis. EMBO J 28, 2100-2113.

Lee, M.S., Han, H.J., Han, S.Y., Kim, I.Y., Chae, S., Lee, C.S., Kim, S.E., Yoon, S.G., Park, J.W., Kim, J.H., et al. (2018). Loss of the E3 ubiquitin ligase MKRN1 represses diet-induced metabolic syndrome through AMPK activation. Nat Commun 9, 3404.

Lee, M.S., Jeong, M.H., Lee, H.W., Han, H.J., Ko, A., Hewitt, S.M., Kim, J.H., Chun, K.H., Chung, J.Y., Lee, C., et al. (2015). PI3K/AKT activation induces PTEN ubiquitination and destabilization accelerating tumourigenesis. Nat Commun 6, 7769.

Leitch, H.G., McEwen, K.R., Turp, A., Encheva, V., Carroll, T., Grabole, N., Mansfield, W., Nashun, B., Knezovich, J.G., Smith, A., et al. (2013). Naive pluripotency is associated with global DNA hypomethylation. Nature structural \& molecular biology 20, 311-316

Leitch, H.G., Surani, M.A., and Hajkova, P. (2016). DNA (De)Methylation: The Passive Route to Naivety? Trends Genet 32, 592-595.

Liu, D., Wang, X., He, D., Sun, C., He, X., Yan, L., Li, Y., Han, J.J., and Zheng, P. (2018). Single cell RNA-sequencing reveals the existence of naive and primed pluripotency in preimplantation rhesus monkey embryos. Genome Res 28, 1481-1493. 
Longshaw, V.M., Baxter, M., Prewitz, M., and Blatch, G.L. (2009). Knockdown of the cochaperone Hop promotes extranuclear accumulation of Stat3 in mouse embryonic stem cells. Eur J Cell Biol 88, 153-166.

Meistermann, D., Bruneau, A., Loubersac, S., Reignier, A., Firmin, J., Francois-Campion, V., Kilens, S., Lelievre, Y., Lammers, J., Feyeux, M., et al. (2021). Integrated pseudotime analysis of human pre-implantation embryo single-cell transcriptomes reveals the dynamics of lineage specification. Cell Stem Cell S1934-5909(21)00185-5.

Messmer, T., von Meyenn, F., Savino, A., Santos, F., Mohammed, H., Lun, A.T.L., Marioni, J.C., and Reik, W. (2019). Transcriptional Heterogeneity in Naive and Primed Human Pluripotent Stem Cells at Single-Cell Resolution. Cell reports 26, 815-824 e814.

Mohammed, H., Hernando-Herraez, I., Savino, A., Scialdone, A., Macaulay, I., Mulas, C., Chandra, T., Voet, T., Dean, W., Nichols, J., et al. (2017). Single-Cell Landscape of Transcriptional Heterogeneity and Cell Fate Decisions during Mouse Early Gastrulation. Cell reports $20,1215-1228$.

Nakamura, T., Fujiwara, K., Saitou, M., and Tsukiyama, T. (2021). Non-human primates as a model for human development. Stem cell reports 16, 1093-1103.

Nakamura, T., Okamoto, I., Sasaki, K., Yabuta, Y., Iwatani, C., Tsuchiya, H., Seita, Y., Nakamura, S., Yamamoto, T., and Saitou, M. (2016). A developmental coordinate of pluripotency among mice, monkeys and humans. Nature 537, 57-62.

Okamoto, I., Patrat, C., Thepot, D., Peynot, N., Fauque, P., Daniel, N., Diabangouaya, P., Wolf, J.P., Renard, J.P., Duranthon, V., et al. (2011). Eutherian mammals use diverse strategies to initiate X-chromosome inactivation during development. Nature 472, 370-374.

Osteil, P., Moulin, A., Santamaria, C., Joly, T., Jouneau, L., Aubry, M., Tapponnier, Y., Archilla, C., Schmaltz-Panneau, B., Lecardonnel, J., et al. (2016). A Panel of Embryonic Stem Cell Lines Reveals the Variety and Dynamic of Pluripotent States in Rabbits. Stem cell reports 7, 383-398.

Peng, G., Suo, S., Chen, J., Chen, W., Liu, C., Yu, F., Wang, R., Chen, S., Sun, N., Cui, G., et al. (2016). Spatial Transcriptome for the Molecular Annotation of Lineage Fates and Cell Identity in Mid-gastrula Mouse Embryo. Dev Cell 36, 681-697.

Petropoulos, S., Edsgard, D., Reinius, B., Deng, Q., Panula, S.P., Codeluppi, S., Plaza Reyes, A., Linnarsson, S., Sandberg, R., and Lanner, F. (2016). Single-Cell RNA-Seq Reveals Lineage and X Chromosome Dynamics in Human Preimplantation Embryos. Cell 165, 10121026.

Pierre, A., Gautier, M., Callebaut, I., Bontoux, M., Jeanpierre, E., Pontarotti, P., and Monget, P. (2007). Atypical structure and phylogenomic evolution of the new eutherian oocyte- and embryo-expressed KHDC1/DPPA5/ECAT1/OOEP gene family. Genomics 90, 583-594.

Piliszek, A., Madeja, Z.E., and Plusa, B. (2017). Suppression of ERK signalling abolishes primitive endoderm formation but does not promote pluripotency in rabbit embryo. Development 144, 3719-3730.

Puschel, B., Daniel, N., Bitzer, E., Blum, M., Renard, J.P., and Viebahn, C. (2010). The rabbit (Oryctolagus cuniculus): a model for mammalian reproduction and early embryology. Cold Spring Harbor protocols 2010, pdb emo139.

Qian, X., Kim, J.K., Tong, W., Villa-Diaz, L.G., and Krebsbach, P.H. (2016). DPPA5 Supports Pluripotency and Reprogramming by Regulating NANOG Turnover. Stem Cells 34, 588-600. 
Ramos-Ibeas, P., Sang, F., Zhu, Q., Tang, W.W.C., Withey, S., Klisch, D., Wood, L., Loose, M., Surani, M.A., and Alberio, R. (2019). Pluripotency and X chromosome dynamics revealed in pig pre-gastrulating embryos by single cell analysis. Nat Commun 10, 500.

Raudvere, U., Kolberg, L., Kuzmin, I., Arak, T., Adler, P., Peterson, H., and Vilo, J. (2019). $\mathrm{g}$ :Profiler: a web server for functional enrichment analysis and conversions of gene lists (2019 update). Nucleic Acids Res 47, W191-W198.

Savatier, P., Osteil, P., and Tam, P.P. (2017). Pluripotency of embryo-derived stem cells from rodents, lagomorphs, and primates: Slippery slope, terrace and cliff. Stem Cell Res 19, 104112.

Schmaltz-Panneau, B., Jouneau, L., Osteil, P., Tapponnier, Y., Afanassieff, M., Moroldo, M., Jouneau, A., Daniel, N., Archilla, C., Savatier, P., et al. (2014). Contrasting transcriptome landscapes of rabbit pluripotent stem cells in vitro and in vivo. Anim Reprod Sci 149, 67-79.

Schoch, C.L., Ciufo, S., Domrachev, M., Hotton, C.L., Kannan, S., Khovanskaya, R., Leipe, D., McVeigh, R., O'Neill, K., Robbertse, B., et al. (2020). NCBI Taxonomy: a comprehensive update on curation, resources and tools. Database (Oxford) 2020.

Setati, M.M., Prinsloo, E., Longshaw, V.M., Murray, P.A., Edgar, D.H., and Blatch, G.L. (2010). Leukemia inhibitory factor promotes Hsp90 association with STAT3 in mouse embryonic stem cells. IUBMB Life 62, 61-66.

Shahbazi, M.N. (2020). Mechanisms of human embryo development: from cell fate to tissue shape and back. Development 147, dev190629.

Shannon, P., Markiel, A., Ozier, O., Baliga, N.S., Wang, J.T., Ramage, D., Amin, N., Schwikowski, B., and Ideker, T. (2003). Cytoscape: a software environment for integrated models of biomolecular interaction networks. Genome Res 13, 2498-2504.

Smith, A. (2017). Formative pluripotency: the executive phase in a developmental continuum. Development 144, 365-373.

Smoot, M.E., Ono, K., Ruscheinski, J., Wang, P.L., and Ideker, T. (2011). Cytoscape 2.8: new features for data integration and network visualization. Bioinformatics 27, 431-432.

Sone, M., Morone, N., Nakamura, T., Tanaka, A., Okita, K., Woltjen, K., Nakagawa, M., Heuser, J.E., Yamada, Y., Yamanaka, S., et al. (2017). Hybrid Cellular Metabolism Coordinated by Zic3 and Esrrb Synergistically Enhances Induction of Naive Pluripotency. Cell metabolism 25, 1103-1117 e1106.

Stirparo, G.G., Boroviak, T., Guo, G., Nichols, J., Smith, A., and Bertone, P. (2018). Integrated analysis of single-cell embryo data yields a unified transcriptome signature for the human preimplantation epiblast. Development 145, dev158501.

Strumpf, D., Mao, C.A., Yamanaka, Y., Ralston, A., Chawengsaksophak, K., Beck, F., and Rossant, J. (2005). Cdx2 is required for correct cell fate specification and differentiation of trophectoderm in the mouse blastocyst. Development 132, 2093-2102.

Stuart, T., Butler, A., Hoffman, P., Hafemeister, C., Papalexi, E., Mauck, W.M., 3rd, Hao, Y., Stoeckius, M., Smibert, P., and Satija, R. (2019). Comprehensive Integration of Single-Cell Data. Cell 177, 1888-1902 e1821.

Takahashi, S., Kobayashi, S., and Hiratani, I. (2017). Epigenetic differences between naive and primed pluripotent stem cells. Cell Mol Life Sci 75, 1191-1203.

Tanaka, T.S., Lopez de Silanes, I., Sharova, L.V., Akutsu, H., Yoshikawa, T., Amano, H., Yamanaka, S., Gorospe, M., and Ko, M.S. (2006). Esg1, expressed exclusively in 
preimplantation embryos, germline, and embryonic stem cells, is a putative RNA-binding protein with broad RNA targets. Dev Growth Differ 48, 381-390.

Tang, F., Barbacioru, C., Bao, S., Lee, C., Nordman, E., Wang, X., Lao, K., and Surani, M.A. (2010). Tracing the derivation of embryonic stem cells from the inner cell mass by single-cell RNA-Seq analysis. Cell Stem Cell 6, 468-478.

Tapponnier, Y., Afanassieff, M., Aksoy, I., Aubry, M., Moulin, A., Medjani, L., Bouchereau, W., Mayere, C., Osteil, P., Nurse-Francis, J., et al. (2017). Reprogramming of rabbit induced pluripotent stem cells toward epiblast and chimeric competency using Kruppel-like factors. Stem Cell Res 24, 106-117.

Teixeira, M., Commin, L., Gavin-Plagne, L., Bruyere, P., Buff, S., and Joly, T. (2018). Rapid cooling of rabbit embryos in a synthetic medium. Cryobiology 85, 113-119.

Tesar, P.J., Chenoweth, J.G., Brook, F.A., Davies, T.J., Evans, E.P., Mack, D.L., Gardner, R.L., and McKay, R.D. (2007). New cell lines from mouse epiblast share defining features with human embryonic stem cells. Nature 448, 196-199.

Trapnell, C., Cacchiarelli, D., Grimsby, J., Pokharel, P., Li, S., Morse, M., Lennon, N.J., Livak, K.J., Mikkelsen, T.S., and Rinn, J.L. (2014). The dynamics and regulators of cell fate decisions are revealed by pseudotemporal ordering of single cells. Nat Biotechnol 32, 381386.

Viebahn, C., Stortz, C., Mitchell, S.A., and Blum, M. (2002). Low proliferative and high migratory activity in the area of Brachyury expressing mesoderm progenitor cells in the gastrulating rabbit embryo. Development 129, 2355-2365.

Wen, J., Zeng, Y., Fang, Z., Gu, J., Ge, L., Tang, F., Qu, Z., Hu, J., Cui, Y., Zhang, K., et al. (2017). Single-cell analysis reveals lineage segregation in early post-implantation mouse embryos. J Biol Chem 292, 9840-9854.

Wianny, F., Real, F.X., Mummery, C.L., Van Rooijen, M., Lahti, J., Samarut, J., and Savatier, P. (1998). G1-phase regulators, cyclin D1, cyclin D2, and cyclin D3: up-regulation at gastrulation and dynamic expression during neurulation. Dev Dyn 212, 49-62.

Zhang, Y., Xiang, Y., Yin, Q., Du, Z., Peng, X., Wang, Q., Fidalgo, M., Xia, W., Li, Y., Zhao, Z.A., et al. (2018). Dynamic epigenomic landscapes during early lineage specification in mouse embryos. Nat Genet 50, 96-105.

Zhao, X.M., Cui, L.S., Hao, H.S., Wang, H.Y., Zhao, S.J., Du, W.H., Wang, D., Liu, Y., and Zhu, H.B. (2016). Transcriptome analyses of inner cell mass and trophectoderm cells isolated by magnetic-activated cell sorting from bovine blastocysts using single cell RNA-seq. Reprod Domest Anim 51, 726-735.

Zhou, W., Choi, M., Margineantu, D., Margaretha, L., Hesson, J., Cavanaugh, C., Blau, C.A., Horwitz, M.S., Hockenbery, D., Ware, C., et al. (2012). HIF1alpha induced switch from bivalent to exclusively glycolytic metabolism during ESC-to-EpiSC/hESC transition. EMBO J 31, 2103-2116.

Zhu, P., Guo, H., Ren, Y., Hou, Y., Dong, J., Li, R., Lian, Y., Fan, X., Hu, B., Gao, Y., et al. (2018). Single-cell DNA methylome sequencing of human preimplantation embryos. Nat Genet 50, 12-19. 


\section{Figure legends}

Figure 1: Transcriptional map of rabbit preimplantation embryo. (A) Experimental design with sample description. E2.7 to E6.6 indicate days of development after coïtum (or insemination); ICM: inner cell mass; PS: primitive streak. (B) Graphical representation of the two first principal components of PCA for the 51 sample transcriptomes generated with bulk RNAseq data. (C) Heatmap representation of the expression level of 48 genes selected based on their known expression in early embryonic lineages of rodents and primates (bulk RNAseq data). (D) Two-dimensional UMAP representations of 17,025 single-cell transcriptomes (left panel) and selected genes (right panels). E3.0-E6.6 indicate the embryonic day (10X Genomics data). (E) Heatmap representation grouping 20 gene markers (from B and C, highlighted in blue, maroon, pink and yellow) with the 20 most differentially expressed genes in TE $v s$. all the other cell types (all), ICM vs. all, EPI vs. all, and PE vs. all (from D). See also Figure S1.

Figure 2: Timing of ICM/TE and EPI/PE segregation. (A) Two-dimensional UMAP representation of 17,025 single-cell transcriptomes showing cells selected for the pseudo-time analysis shown in B and C. (B,C) Pseudo-time analysis of selected cells (shown in A), organized by embryonic day (B) and lineage assignment (C), respectively. (D) Heatmap representation of the 97 most differentially expressed genes between morula, ICM and TE (from pseudo-time analysis). (E) Immunofluorescence analysis of SOX2 and TFAP2C expression in E3.5 $(n=7)$ and E4.0 $(n=4)$ blastocysts. Merge pictures include DAPI staining. Scale bars: $100 \mu \mathrm{m}$. (F) KEGG pathways enrichment analysis from the 500 most upregulated genes in ICM vs. TE at E3.5/E4.0 (top panel) and the 500 most up-regulated genes in TE vs. ICM at E3.5/E4.0 (bottom panel). (G) Two-dimensional UMAP representation of 17,025 single-cell transcriptomes showing cells selected for pseudo-time analysis shown in $\mathrm{H}$ and I. (H,I) Pseudo-time representations of selected cells (shown in G), organized by embryonic day $(\mathbf{H})$ and lineage assignment $(\mathbf{I})$, respectively. (J) Heatmap representation of the 89 most differentially expressed genes between morula, EPI and PE (from pseudo-time analysis). (K) Immunofluorescence analysis of SOX2, OCT4, and GATA6 at E4.0 (n = 4) and E5.0 $(n=4)$. Merge pictures include DAPI staining. Scale bars: $100 \mu \mathrm{m}$ at E4.0 and $200 \mu \mathrm{m}$ at E5.0. (L) KEGG pathways enrichment analysis from the 500 most up-regulated genes in EPI $v s$. PE at E4.0/E5.0 (top panel) and the 500 most up-regulated genes in PE vs. EPI at E4.0/E5.0 (bottom panel). See also Figure S2. 
Figure 3: Late epiblast diversification and PGC segregation. (A) Two-dimensional UMAP representation of 17,025 single-cell transcriptomes showing cells selected for pseudo-time analysis shown in B-D. (B,C) Pseudo-time representation of selected cells (shown in A), showing embryonic day (B) and lineage assignment (C), respectively. (D) Heatmap representation of the 100 most differentially expressed genes between EPI at E5.0 and EPI at E6.0/E6.6 (EPIa, EPIb, EPIc, and EPId). (E-H) Immunofluorescence detection of SOX2, TFAP2C, SOX17, and TBXT in E6.6 (E,F; n = 5) (Scale bars : $400 \mu \mathrm{m}, 100 \mu \mathrm{m}$, and $50 \mu \mathrm{m}$ depending on enlargements) and E6.0 (G,H; n = 3) (Scale bars : $200 \mu \mathrm{m}$ and $100 \mu \mathrm{m}$ for enlargements) epiblasts. Merge pictures include DAPI staining. (I) Dot plot representation of single cells expressing TFAP2C, PRDM1/BLIMP1, NANOS3 and SOX17 in the 10X Genomics dataset. Colors indicate clusters EPI_5.0, or EPIa to EPId. See also Figure S3.

Figure 4: Transcriptome map of the pluripotency continuum. (A) Heatmap representation of the expression level of 30 genes selected on the basis of their expression in morula, ICM and EPI_ant of rodents and primates (bulk RNAseq). (B) Heatmap representation grouping 20 markers (from A) with the 20 most differentially expressed genes (10X Genomics "PLURI dataset") in ICM vs. all, EPI-mid vs. all, EPI-ant vs. all, and PS vs. all. (C) Immunofluorescence detection of ESRRB, SOX2, OTX2, FOLR1, GATA6, and CD57 in E4.0 $(n=23)$ and E6.6 $(n=16)$ embryos. Merge pictures include DAPI staining. Scale bars: $50 \mu \mathrm{m}$. (D) Violin plot representation of gene expression calculated from the "PLURI dataset." $1^{\text {rst }}, 2^{\text {nd }}$ and $3^{\text {rd }}$ rows show expression of naïve, formative and primed pluripotency gene markers, respectively. (E) Violin plot representation of gene expression measured by Biomark qRT-PCR. See also Figure S4.

Figure 5: Epigenetic characterization of the pluripotency continuum. (A) Immunofluorescence detection of 5-methylcytosine $(5 \mathrm{meC})$ and 5-hydroxymethylcytosine $(5 \mathrm{hmeC})$ in E3.0 morula $(\mathrm{n}=16)$, E3.5 $(\mathrm{n}=18), \mathrm{E} 4.0(\mathrm{n}=22)$ and E5.0 $(\mathrm{n}=25)$ blastocysts, E6.0 $(n=6)$ and E6.6 $(n=5)$ epiblasts. ICM and epiblast are surrounded by dotted lines. Merge images include propidium iodide staining in blue. Scale bars: $50 \mu \mathrm{m}$. (B) Violin plot representations of DNMT3A, DNMT3B, and TET2 expression ("PLURI dataset"). (C) Immunolabeling of histone 2A lysine119 ubiquitinated (H2AK119ubi) marks in embryos at E3.0 to E6.6 $(\mathrm{n}=41)$ (scale bar: $50 \mu \mathrm{M})$. Enlargements show single nuclei (scale bar: 12.5 
$\mu \mathrm{m})$. (D) Immunolabeling of histone 3 lysine 27 tri-methylation (H3K27me3) marks in embryos at E5.0-E6.6 $(\mathrm{n}=12$ ) (scale bar: $50 \mu \mathrm{m}$ ). Enlargements show single nuclei (scale bar: $12.5 \mu \mathrm{m}$ ). (E) Heatmap representation of bulk RNAseq dataset showing the expression of 25 genes involved in DNA methylation and polycomb repressive complexes. (F) Violin plot representation of the average expression of genes located on the $\mathrm{X}$ chromosome compared to those located on the autosomes 2, 3, 4 and 5, calculated from the 10X Genomics data at E3.0 to E6.6. See also Figure S5.

Figure 6: Metabolic characterization of the pluripotency continuum. (A) Heatmap representation of the bulk RNAseq dataset and showing the expression of genes involved in glycolysis and oxidative phosphorylation (OXPHOS). NC, nuclear genome; MT mitochondrial genome in morula (E2.7), TE (E3.5-E6.6), ICM (E3.5/E4.0), EPI_late (E6.0E6.6) and PE_late (E6.0). (B) Violin plot representation of the expression of genes involved in OXPHOS and glycolysis at different embryonic stages in the "PLURI dataset". (C) Metabolism analysis of live rabbit embryos between E3.0 and E6.6. The $1^{\text {st }}$ vertical row show images of embryos ( $\mathrm{n}=34,5$ to 11 embryos per stage) treated with both tetramethylrhodamine ethyl ester (TMRE, red staining) and Hoechst (blue). The $2^{\text {nd }}$ vertical row shows enlargements of ICM/epiblast cells after TMRE staining. The $3^{\text {rd }}$ vertical row show images of embryos $(n=90,5$ to 19 embryos per stage) treated with GluUptake (yellow staining) and Hoechst (blue). The $4^{\text {th }}$ vertical row shows enlargements of ICM/epiblast cells after GluUptake staining. Scale bars: $50 \mu \mathrm{m}$, and $20 \mu \mathrm{m}$ for enlargements. (D) Images of rabbit embryos (stages as indicated; $\mathrm{n}=52,6$ to 12 embryos per stage) treated with Image-iT Hypoxia reagent to reveal HIF-1 $\alpha$ activity. Images in the top row show co-staining of cytoplasm with iT Hypoxia reagent (yellow) and of nucleus with DAPI (blue); Images in the bottom row show enlargement of ICM/epiblast cells after treatment with iT Hypoxia reagent. Scale bars: $50 \mu \mathrm{m}$ and $20 \mu \mathrm{m}$ for enlargements. The violin plot represents $H I F-1 a$ expression (stages as indicated; "PLURI dataset"). See also Figure S5.

Figure 7: Defining markers of naïve pluripotency in rabbits. (A) Venn diagram representation of the total numbers of differentially expressed genes between ICM (E3.5/E4.0), TE (E3.0-E6.6), EPI_late (E6.0-E6.6), and PE_late (E6.0) in the bulk RNAseq dataset. (B) Volcano plot representations of the 3237 differentially expressed genes between ICM (E3.5/E4.0) and other samples described in A. (C) Gene Ontology biological process 
analysis of the significantly differentially expressed genes (log Fold Change $<-0.5$ and $\log$ Fold Change > 0.5) between ICM (E3.5/E4.0) and other three samples described in A, with genes up-regulated in orange and down-regulated in blue. (D) KEGG pathway analysis of the 3237 differentially expressed genes described in $\mathbf{A}$, with genes up-regulated in orange and down-regulated in blue. (E) Dot plot representation (-log p-value as a function of log foldchange) of the 140 differentially expressed genes between ICM (E3.5/E4.0) and other samples in the single-cell RNAseq dataset. (F) GO biological process analysis of the 140 up-regulated genes in ICM/EPI described in E. (G) Violin plot representation of KDM4A expression in morula/ICM/EPI cells at E3.0 to E6.6 ("PLURI dataset”). (H) Immunofluorescence analysis of KDM4A expression in E4.0 $(n=4)$ and E6.0 $(n=4)$ rabbit embryos and counterstained with DAPI. Scale bars: $50 \mu \mathrm{m}$. (I) Cytoscape representation of Gene Set Enrichment Analysis (GSEA) of the differentially expressed genes in ICM/EPI (E3.5/E4.0) versus other samples showing KEGG terms enriched (in red) or depleted (in blue) in ICM. (J) Dot plot representation (-log p-value as a function of log fold-change) of the positively differentially expressed genes between ICM (E3.5/E4.0) and other cell types in mice (Argelaguet et al., 2019). (K) Dot plot representation (-log p-value as a function of log fold-change) of the positively differentially expressed genes between ICM/EPI (E6.0/E9.0) and other cell types in cynomolgus monkey (Nakamura et al., 2016). (L) Venn diagram representation of the 120 upregulated genes in rabbit ICM/EPI described in $\mathbf{E}$ between rabbits, mice and cynomolgus monkeys. See also Figures S6 and S7). 


\section{Figure 1: Transcriptional map of rabbit preimplantation embryo.}

A

Bulk RNAseq

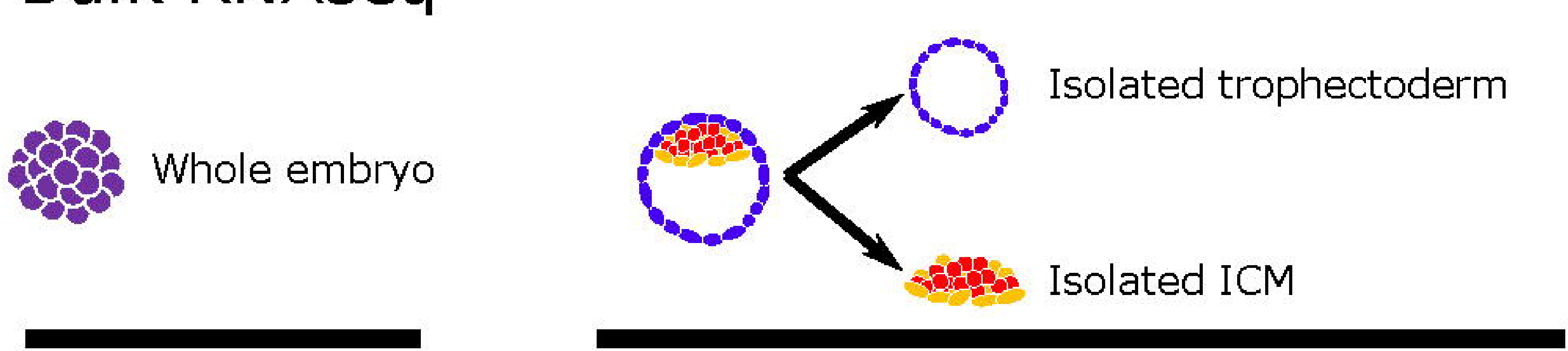

E2.7-3.0

Morula
E3.5

Early blastocyst
E4.0

Mid blastocyst
E5.0

Late blastocyst

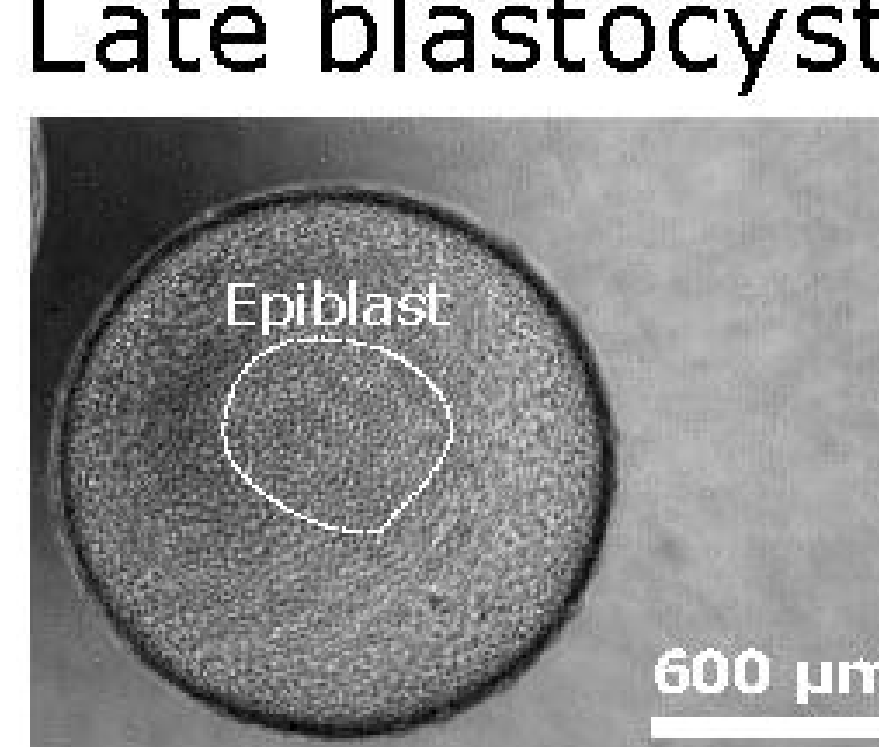

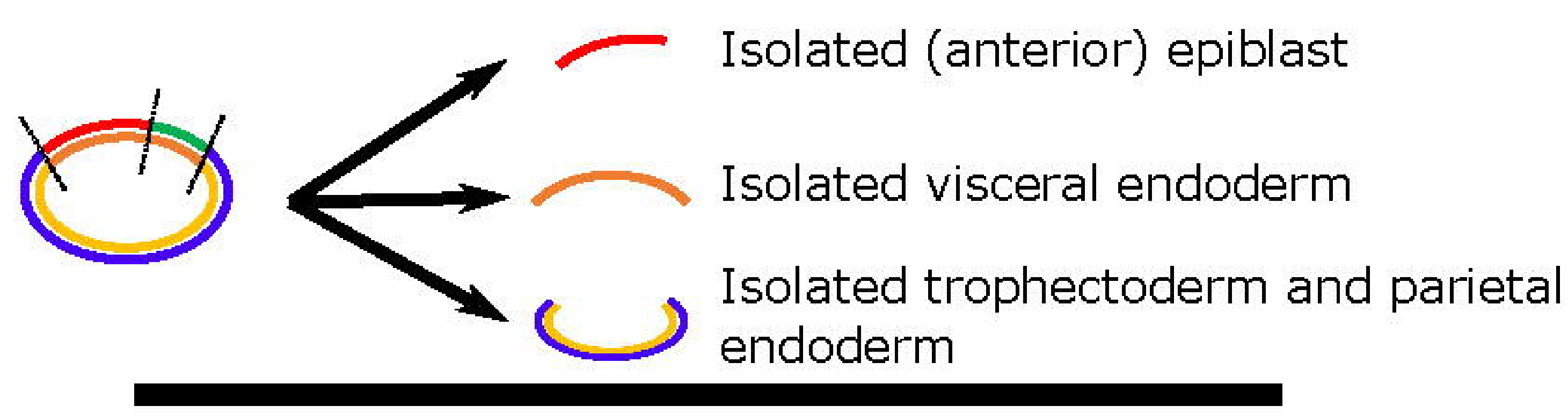

E6.0

Expanded blastocyst

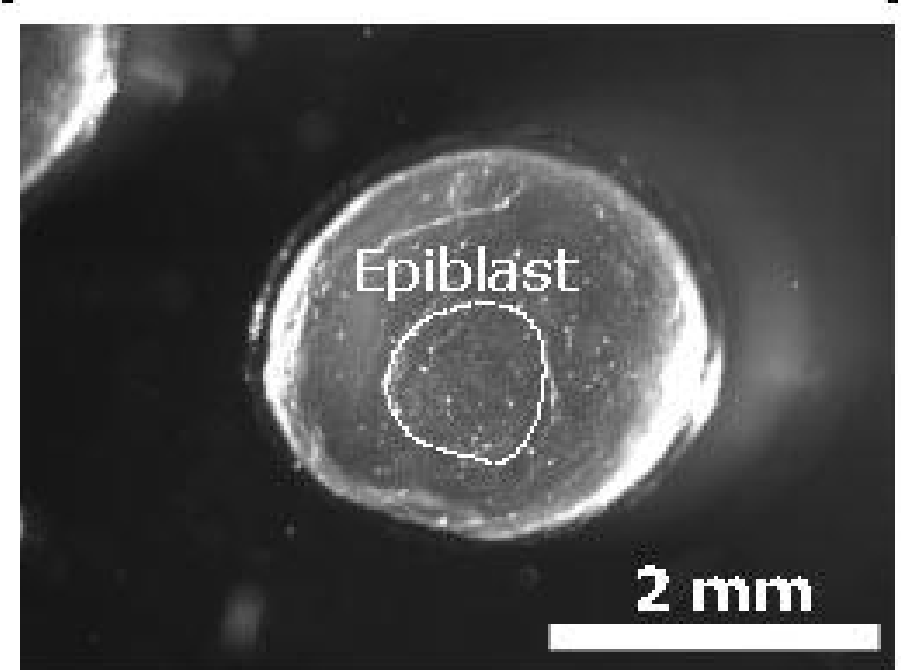

E6.3-6.6

Early gastrula

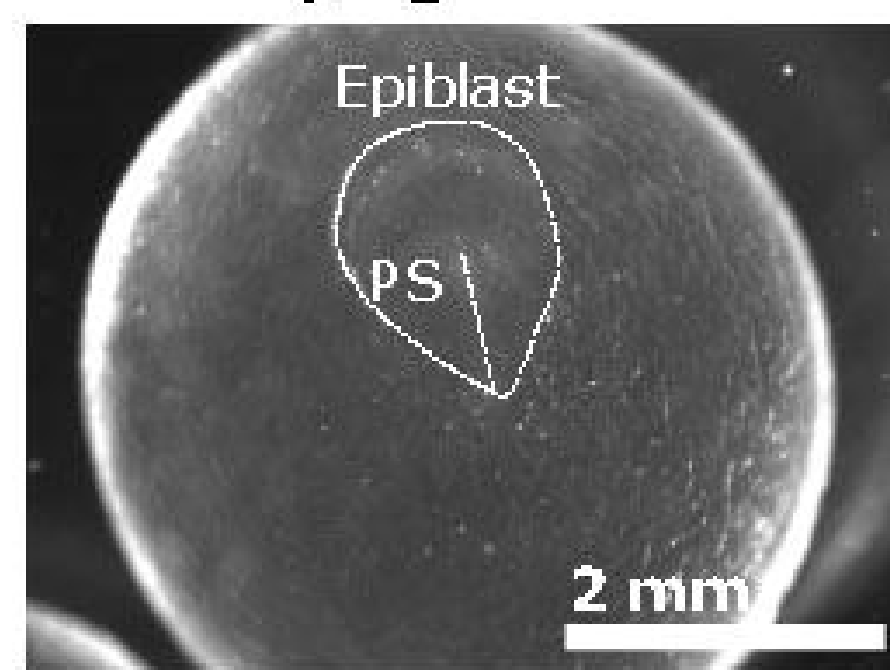

Single-cell RNAseq (10X Genomics) \& Biomark

B

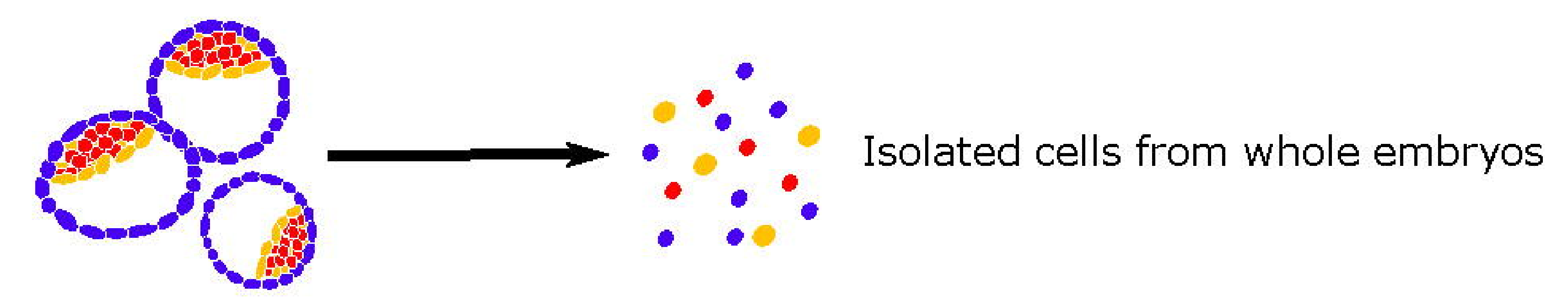

D

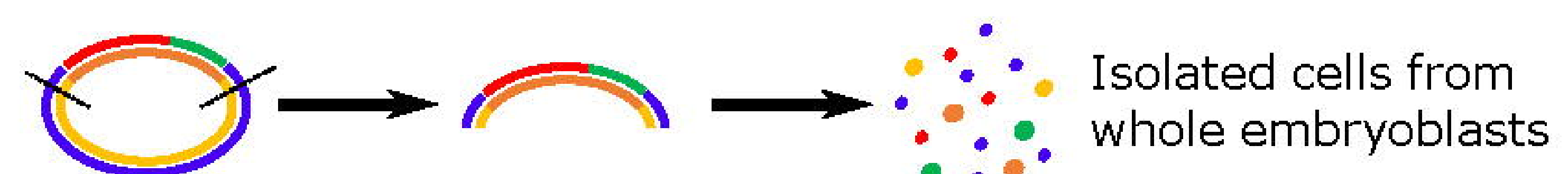

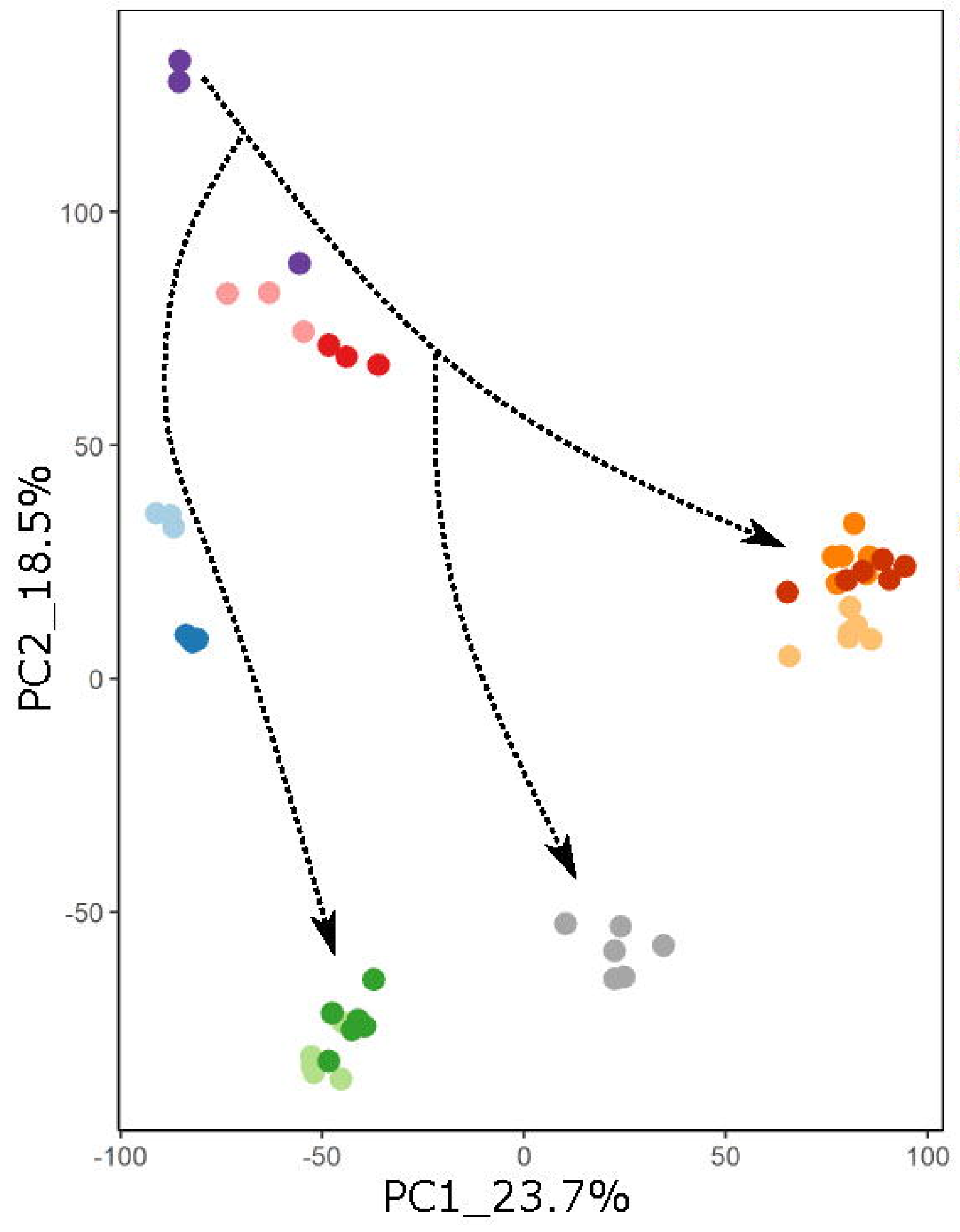

Morula

- ICM_3.5

- TE 3.5

- TE 4.0

- TE_6.0

- TE-6.6

- PE_6.0

- EPI_6.0

- EPI_ant_6.3

c

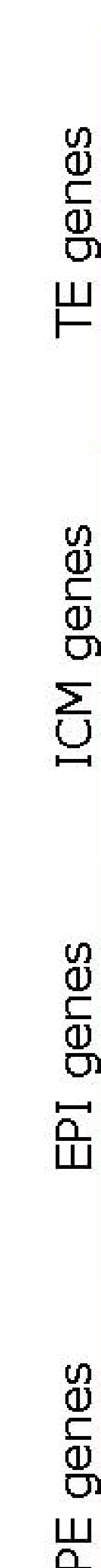
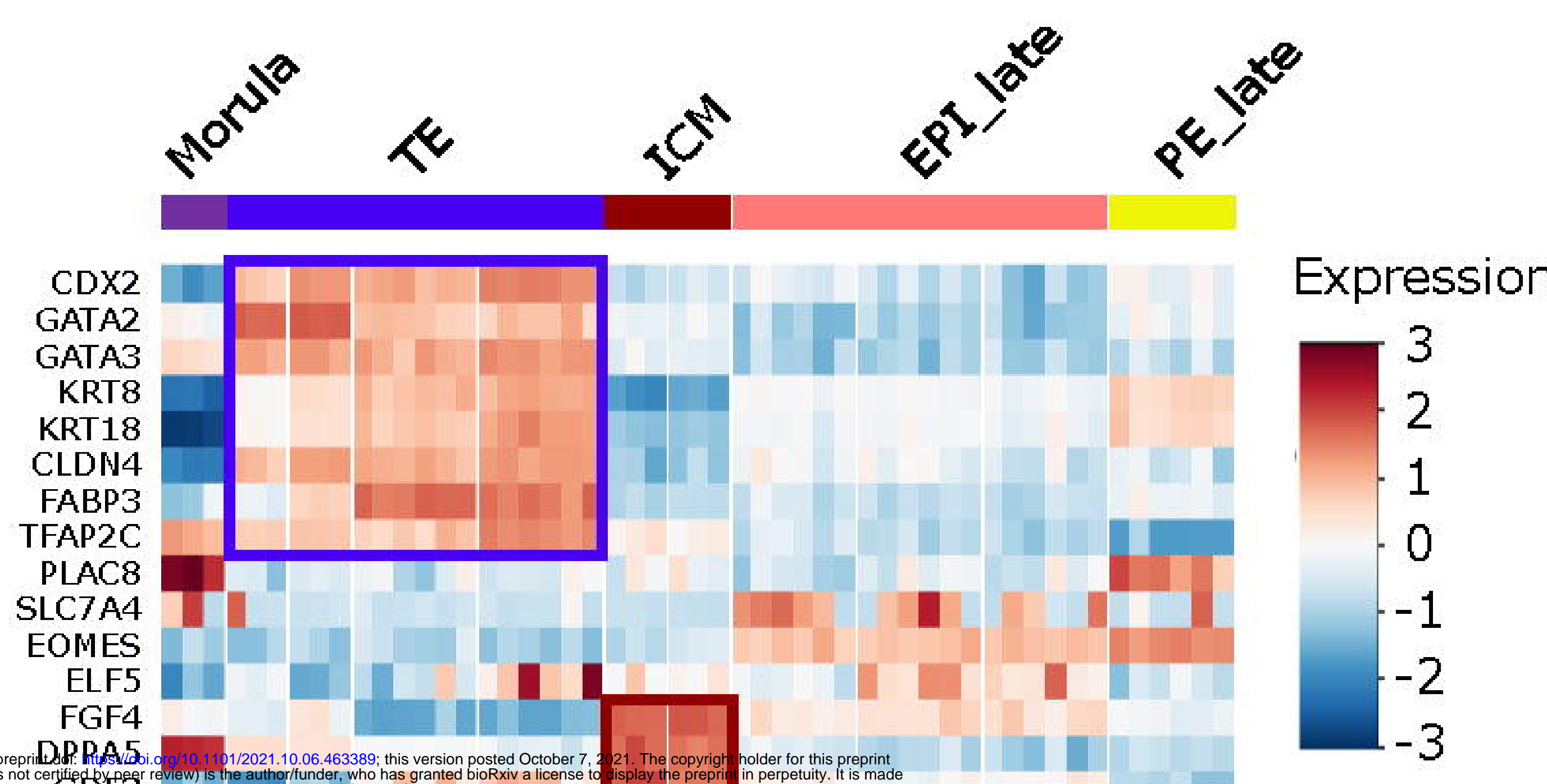

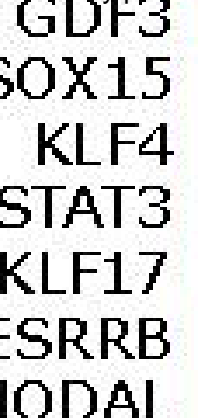

年
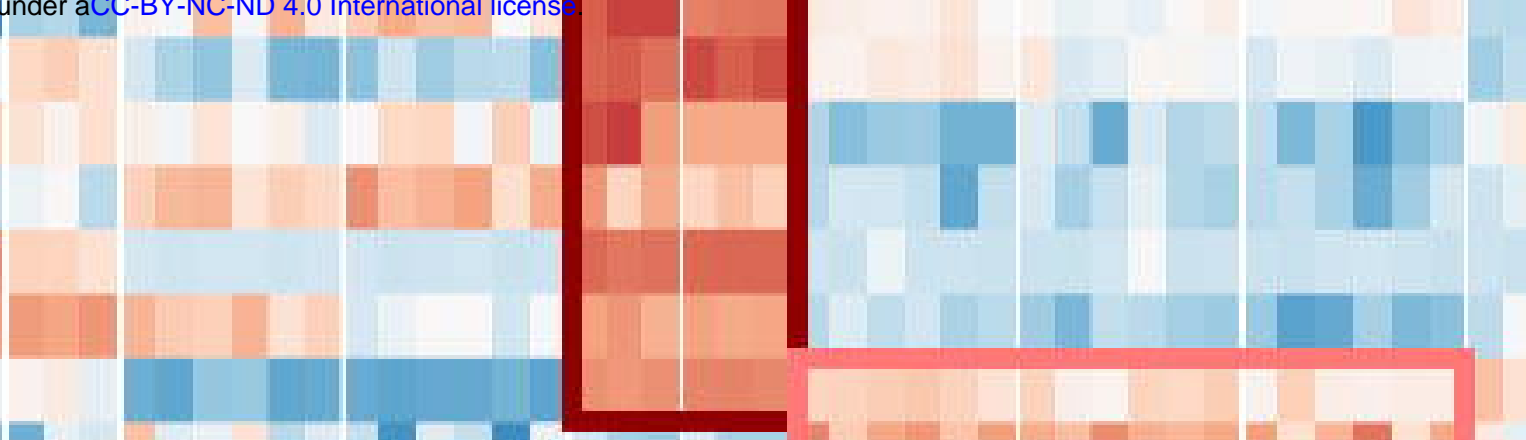

1. 10.
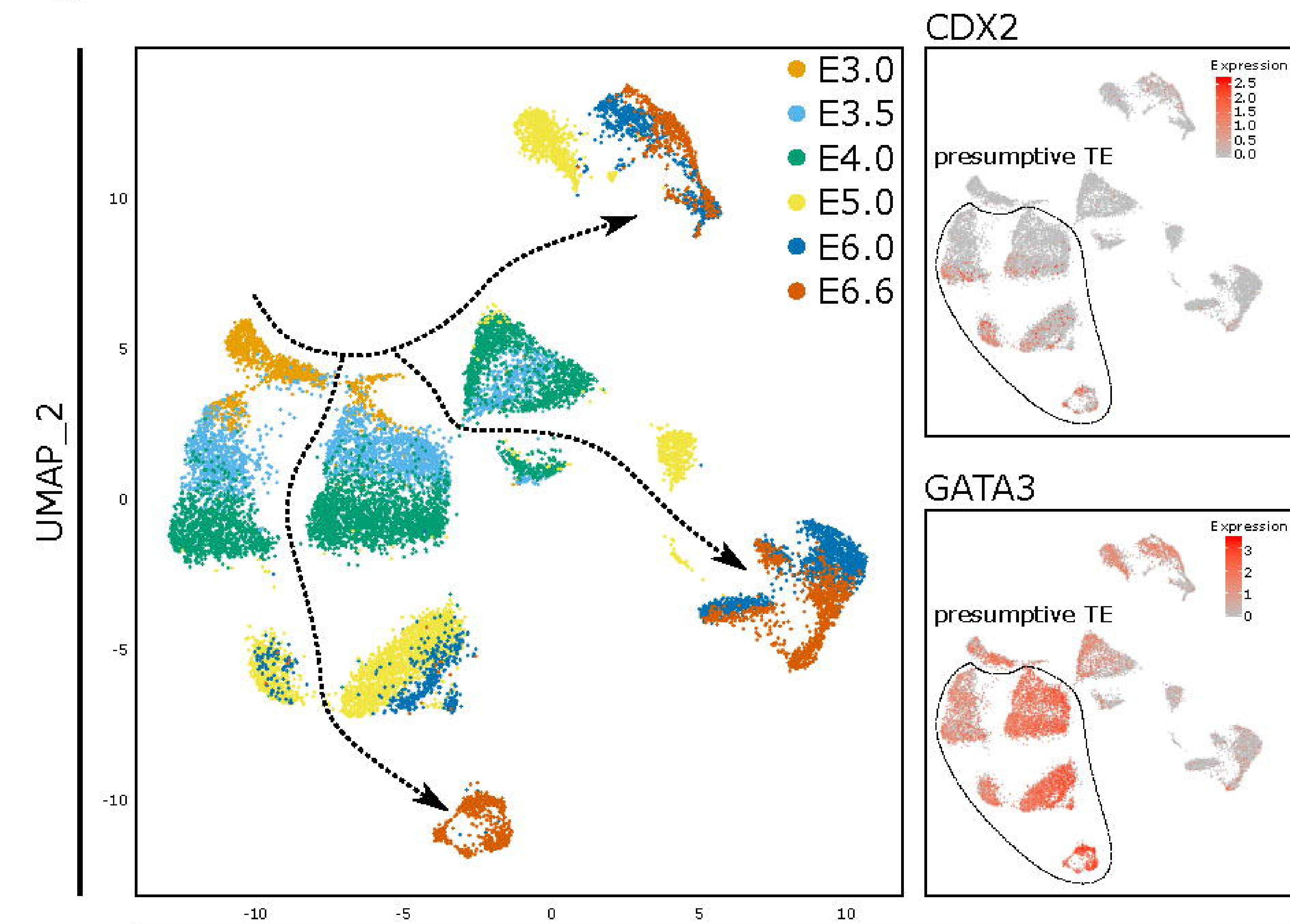
POU5F1

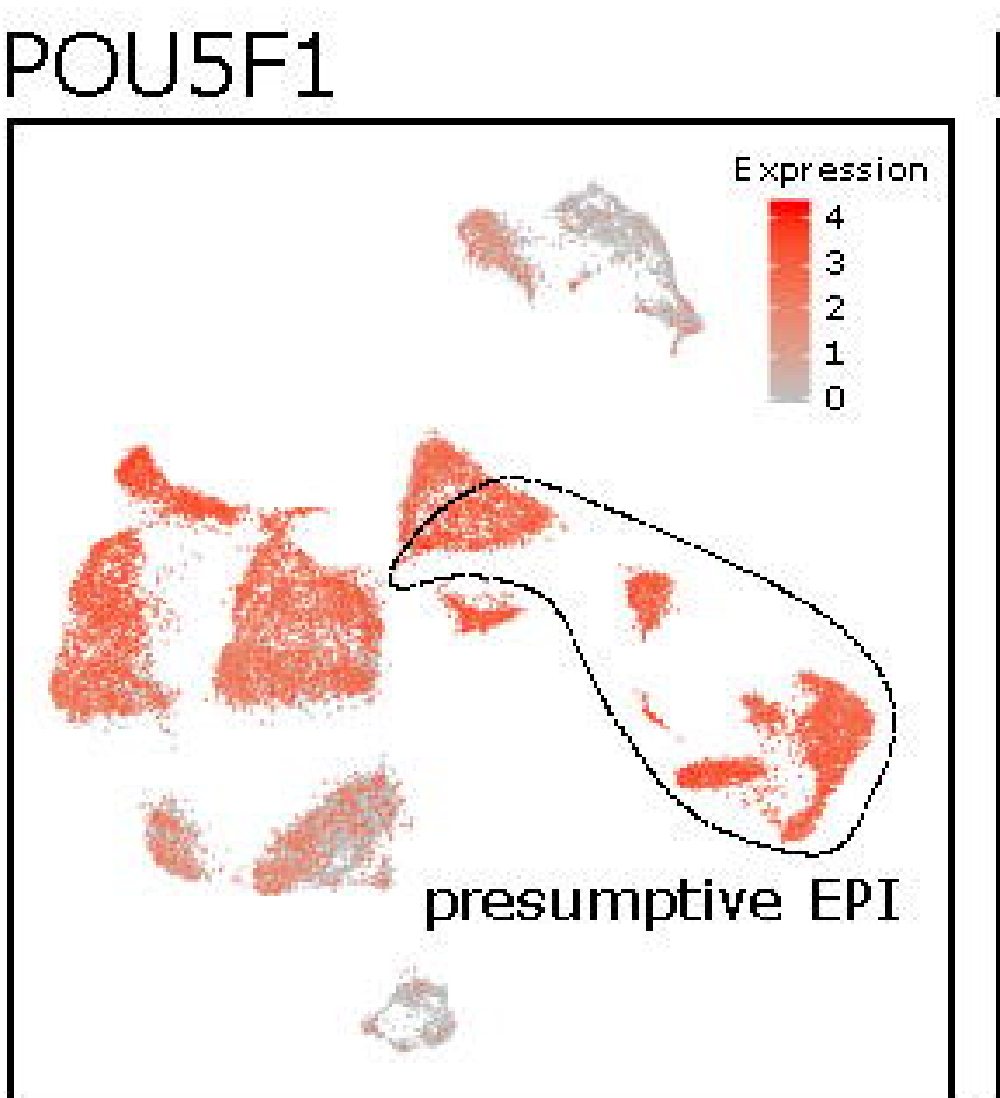

PDGFRA

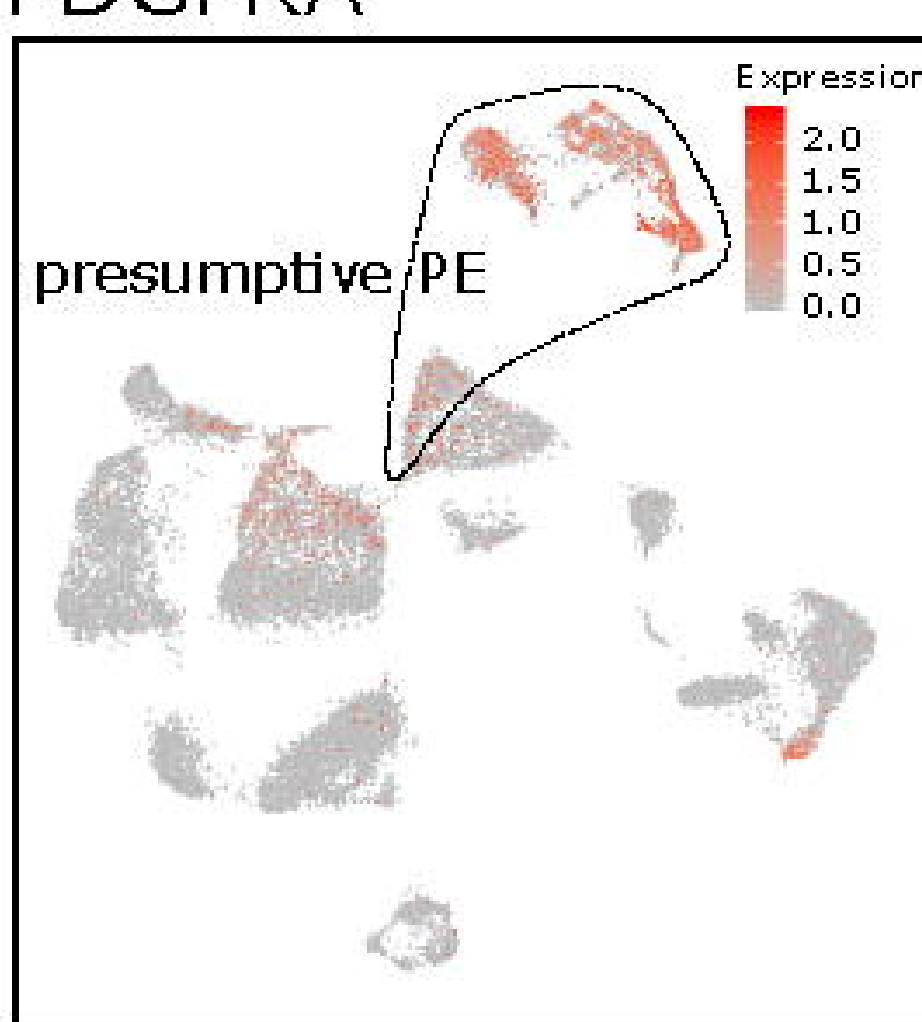

GATA3

$\mathrm{SOX} 2$

HNF1B
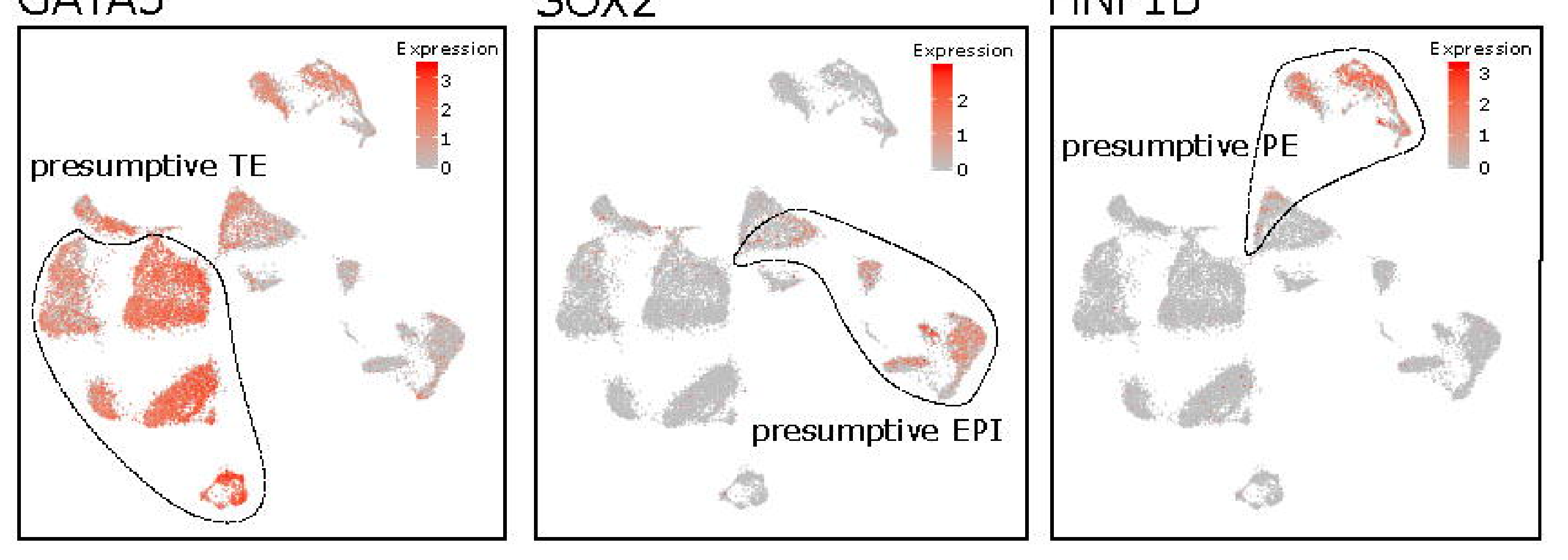

UMAP_1

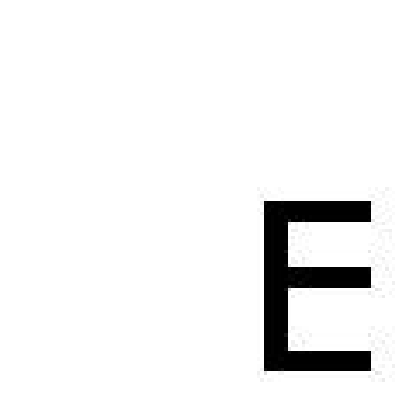

E
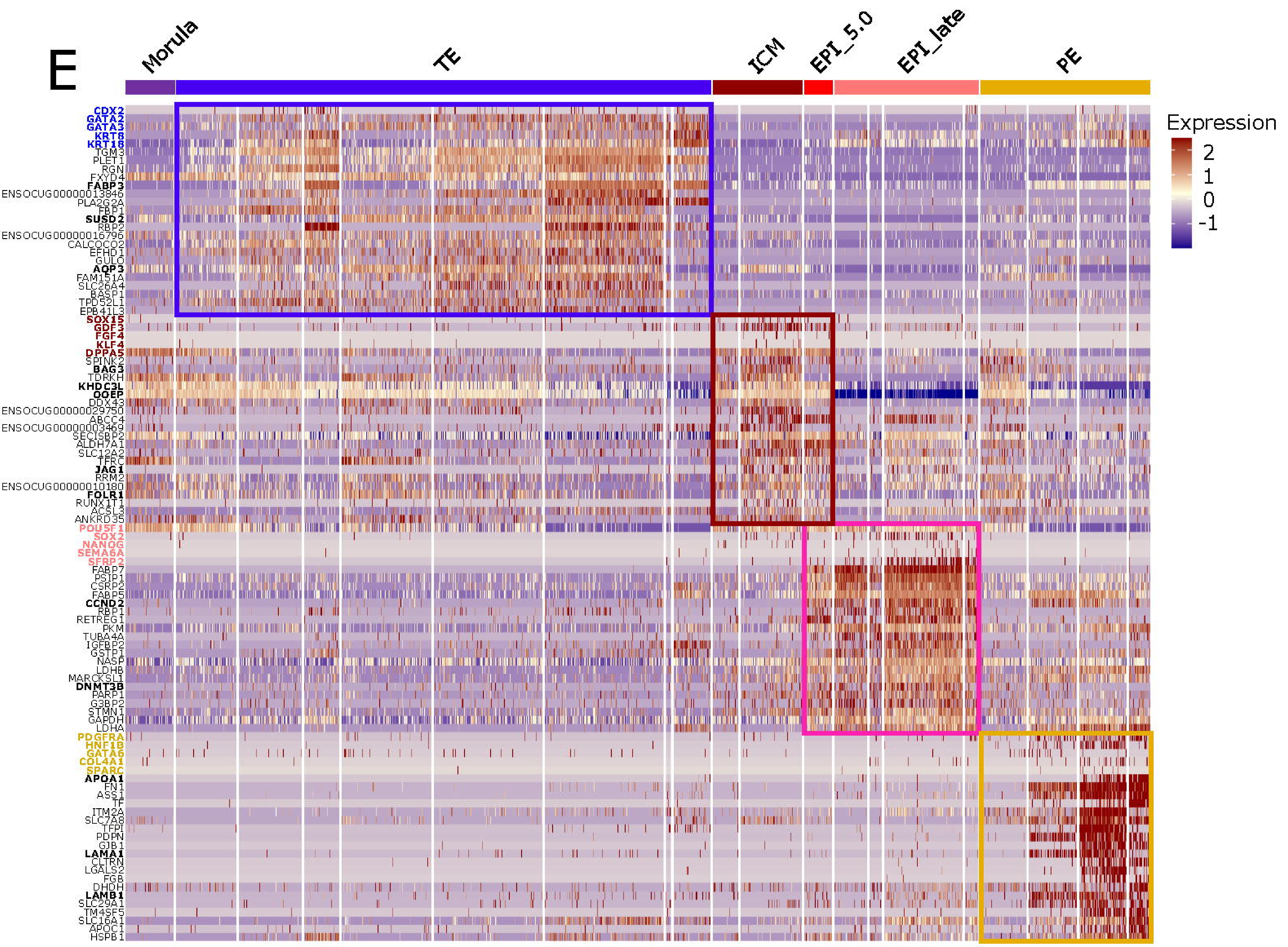
Figure 2: Timing of ICM/TE and EPI/PE segregation.

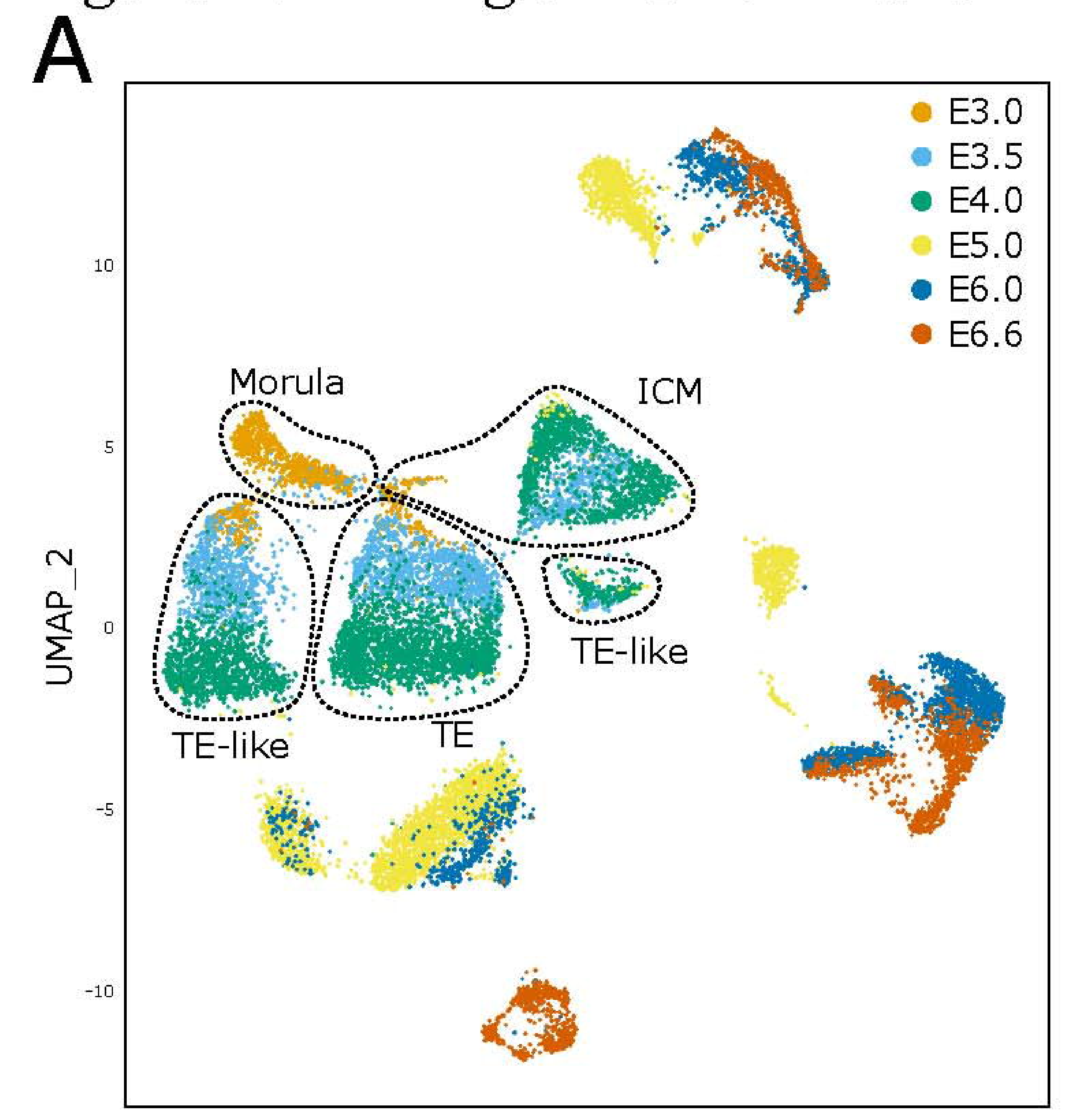

UMAP_1

\section{$\mathrm{E}$}
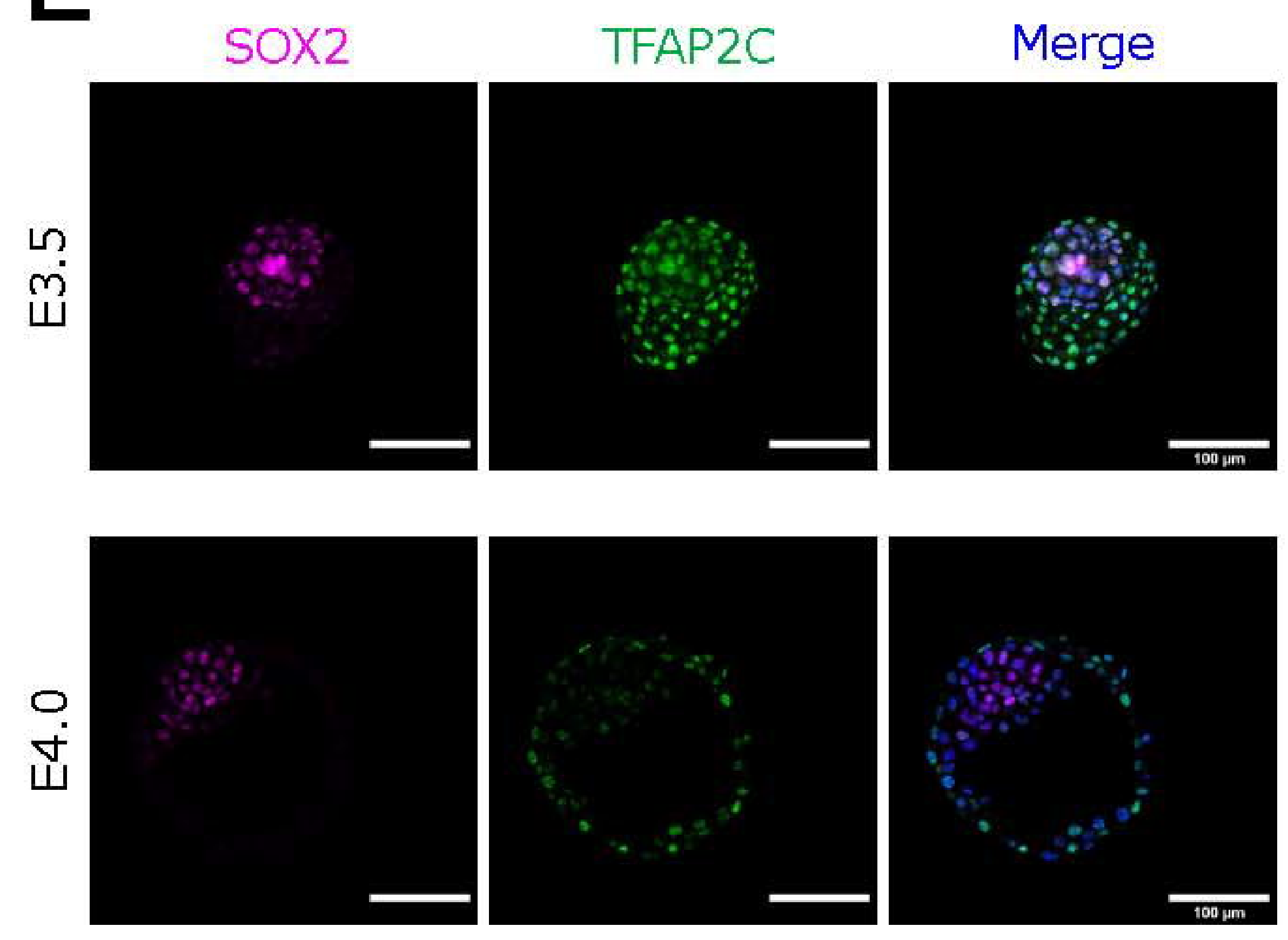

G
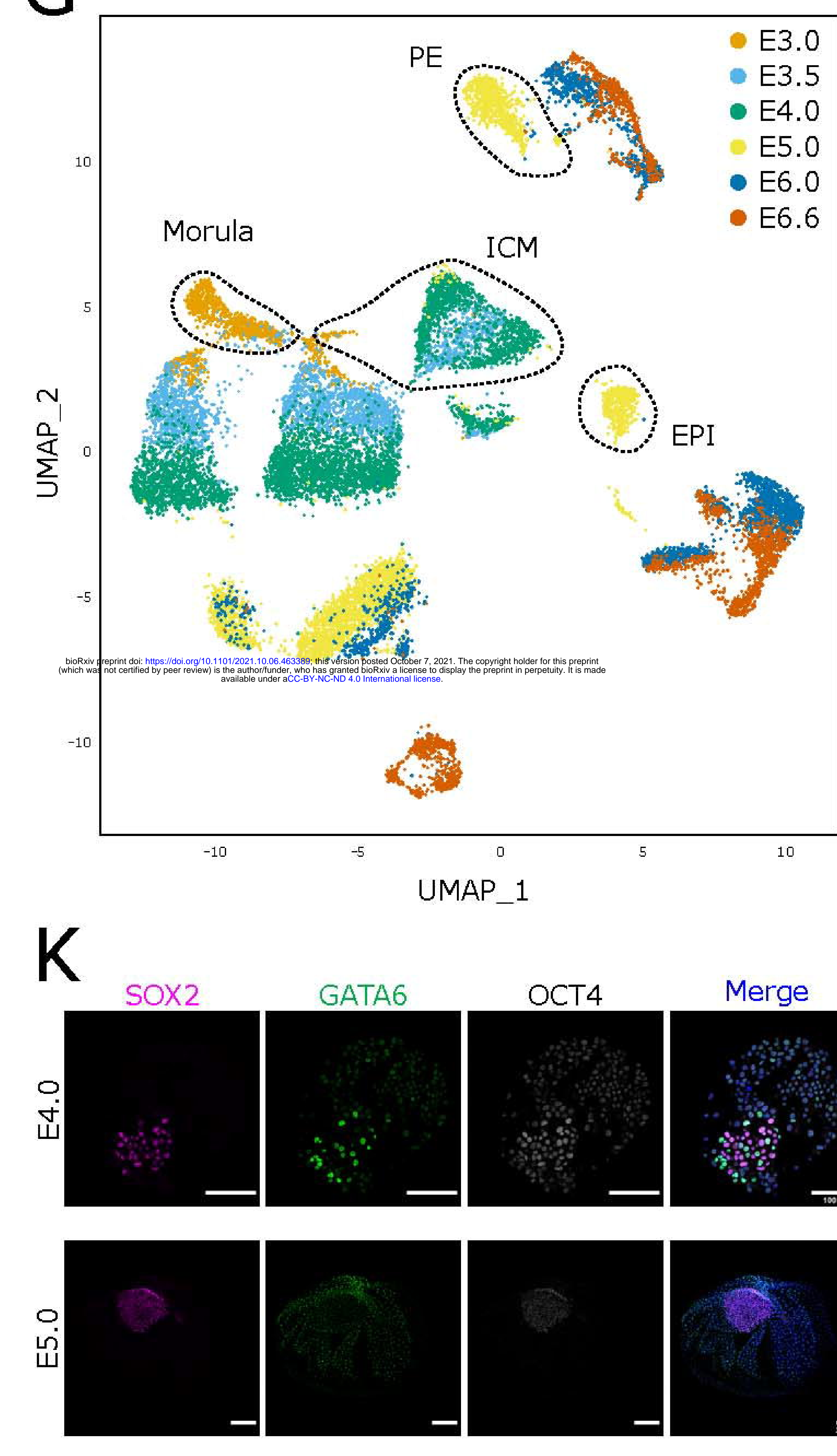

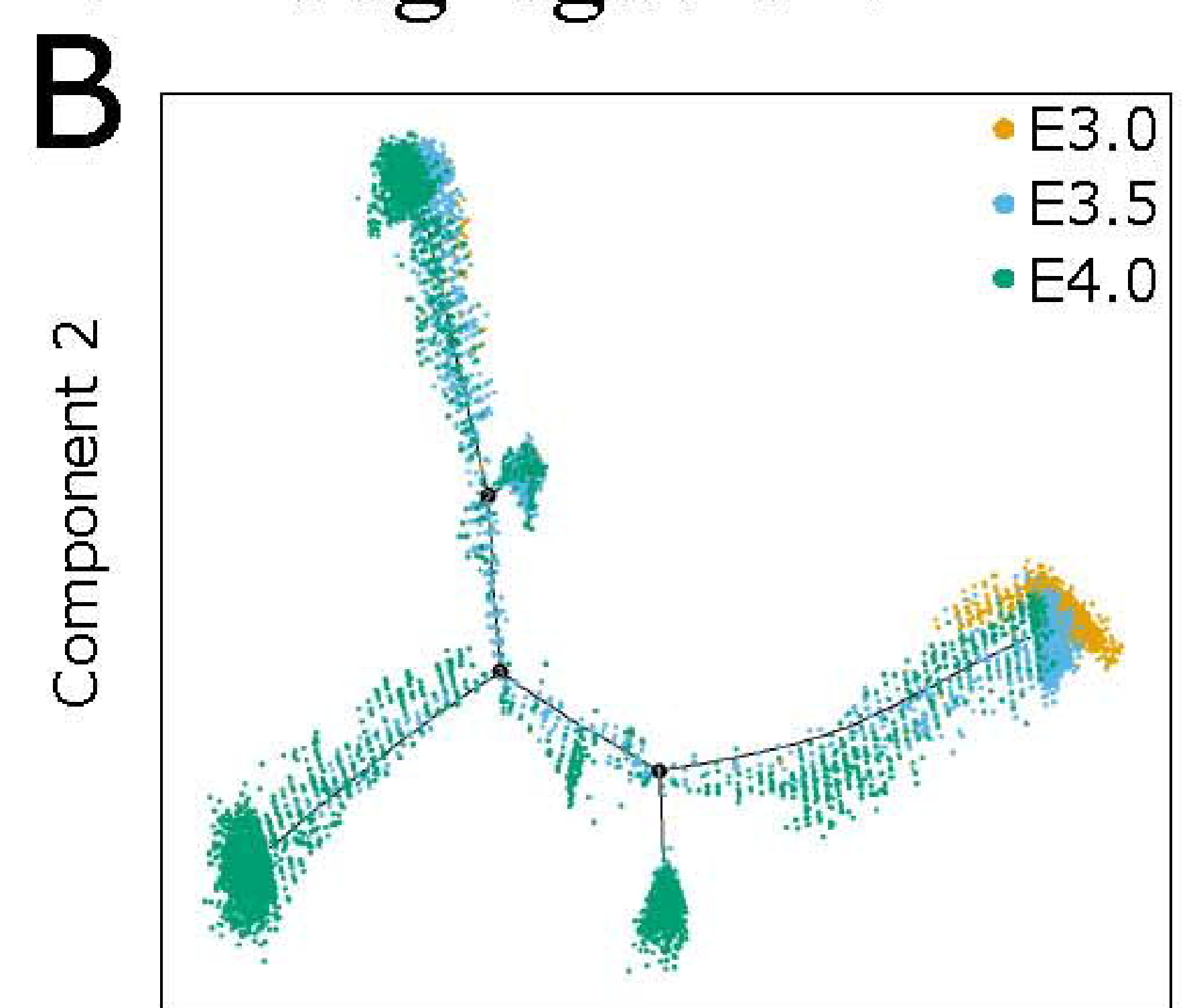

C

F KEGG Pathways

Negative $\log 10$ of the adjusted p-value

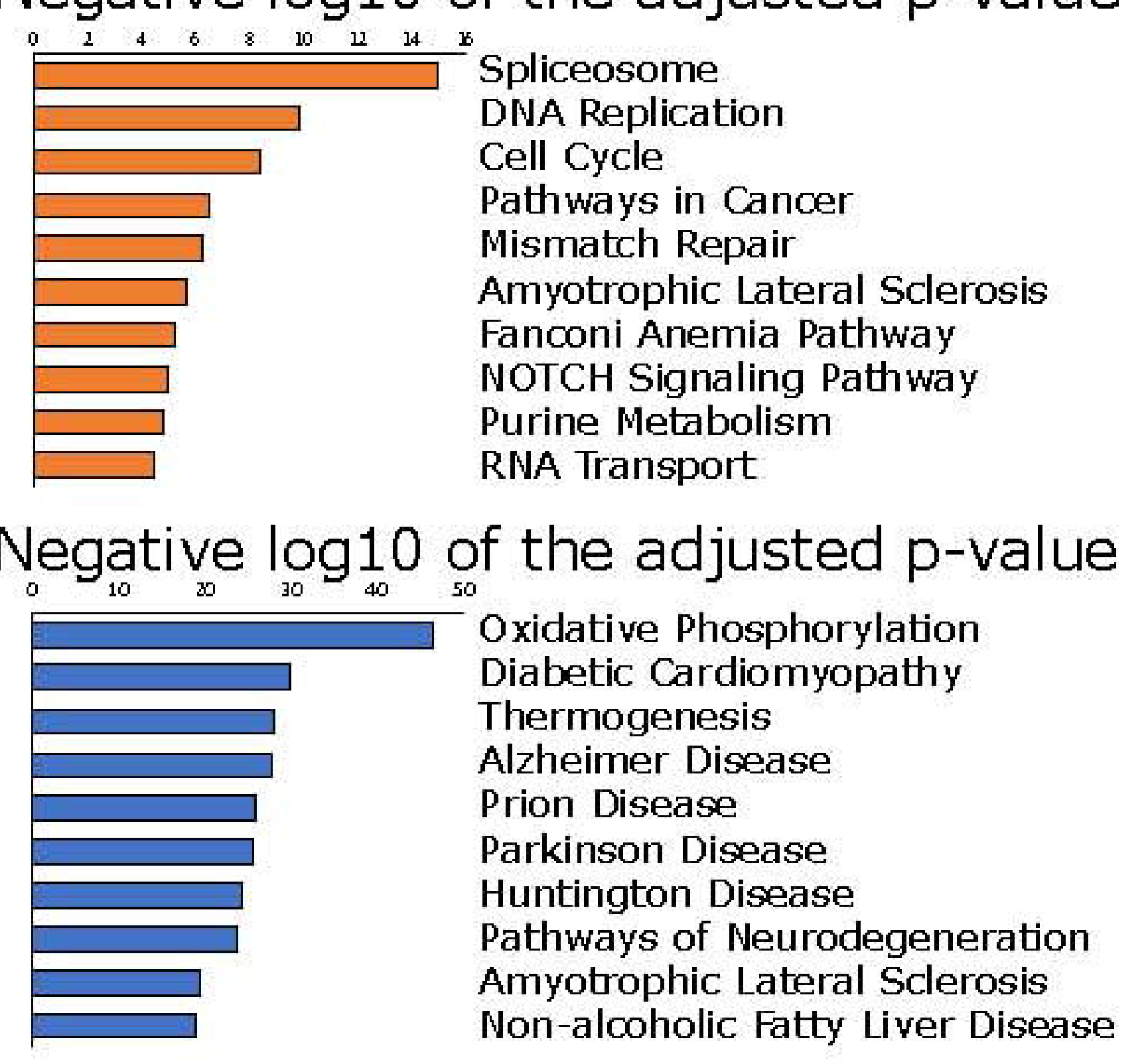

$\mathrm{H}$

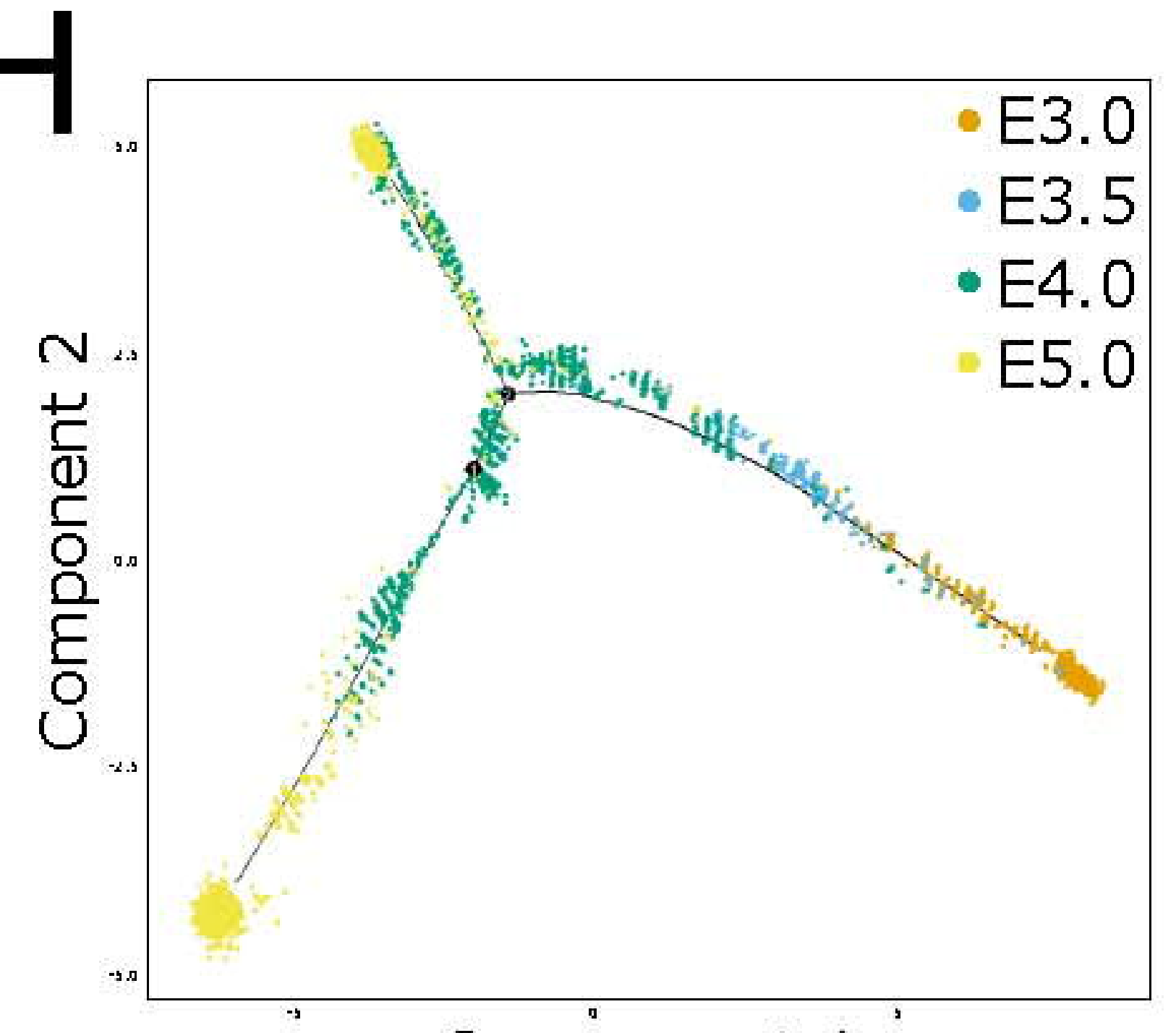

Component 1

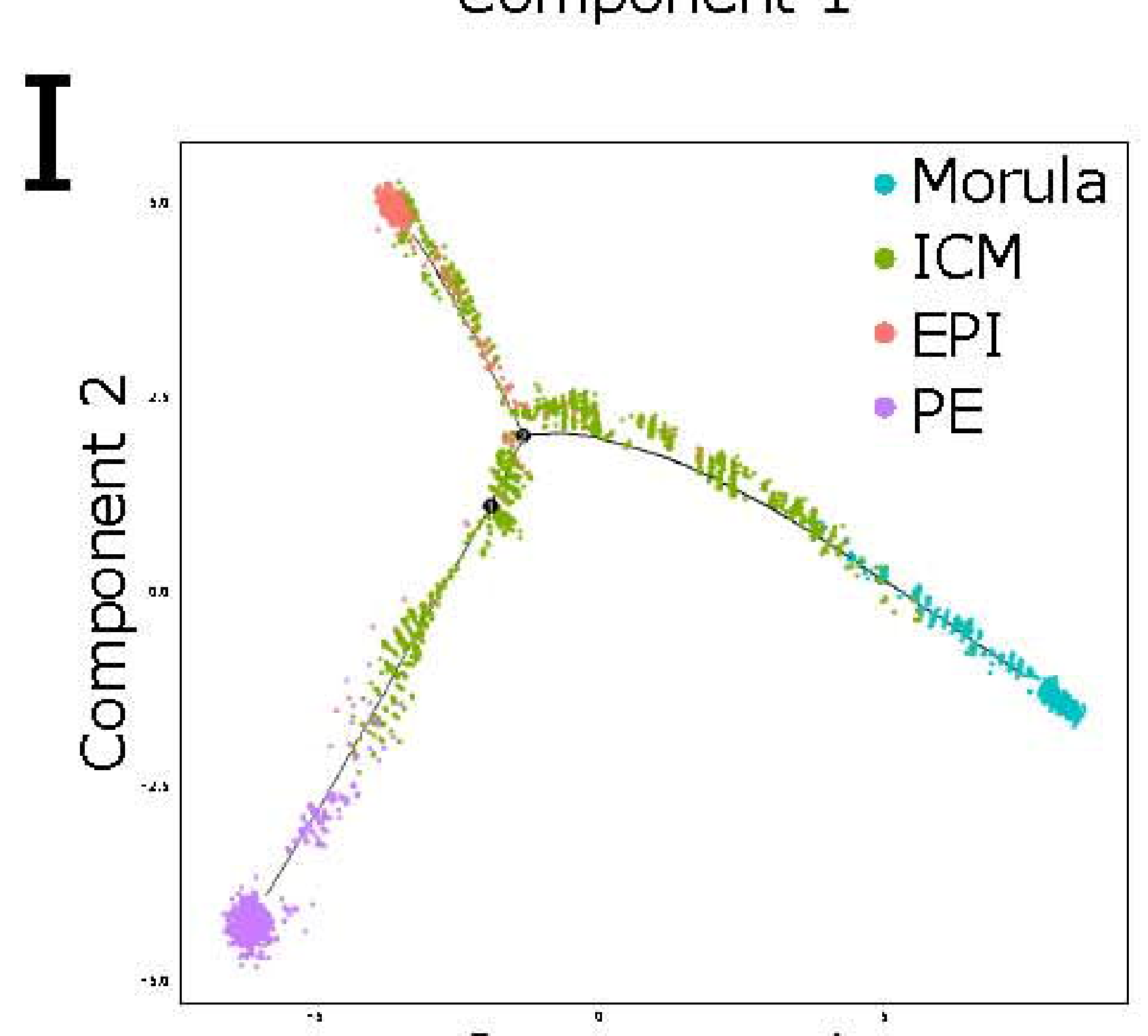

Component 1

L KEGG Pathways

Negative $\log 10$ of the adjusted p-value

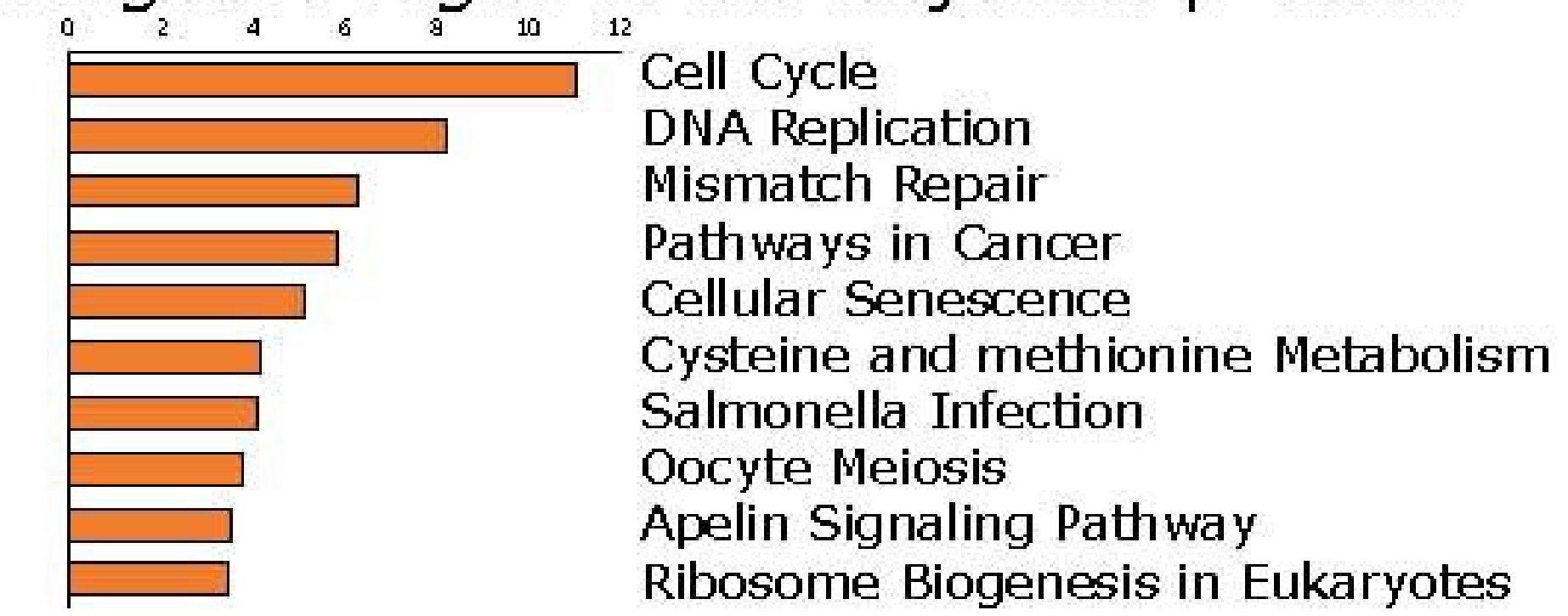

Negative $\log 10$ of the adjusted p-value

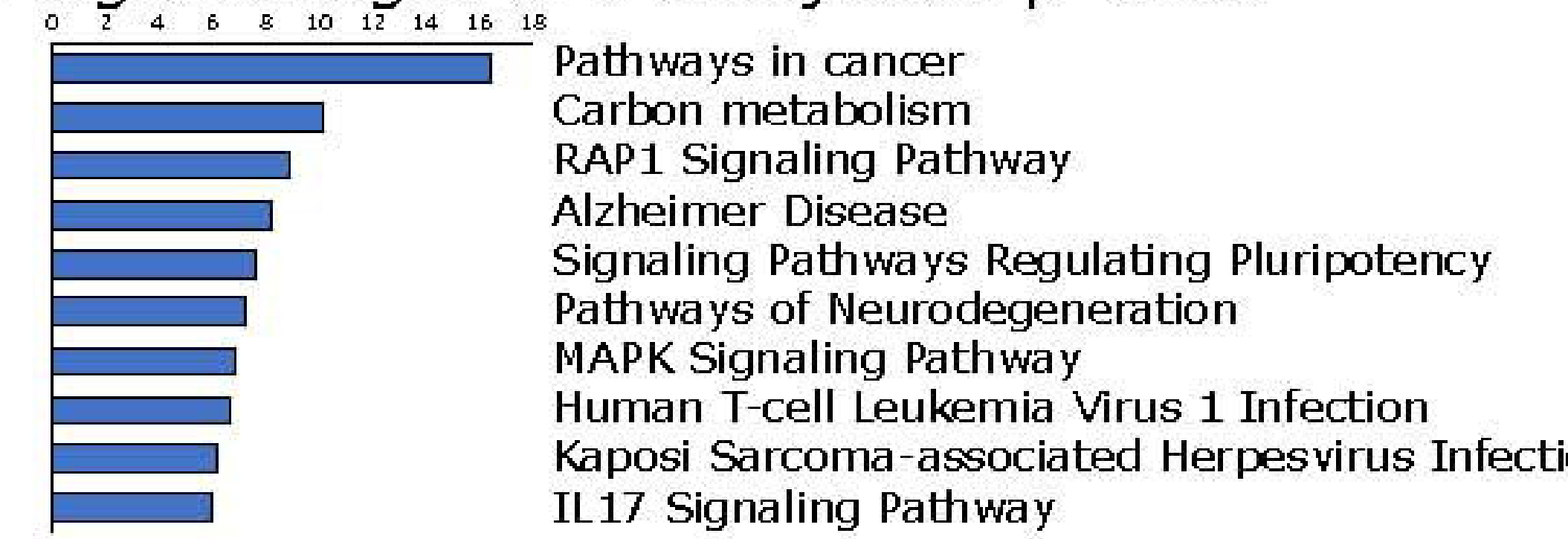

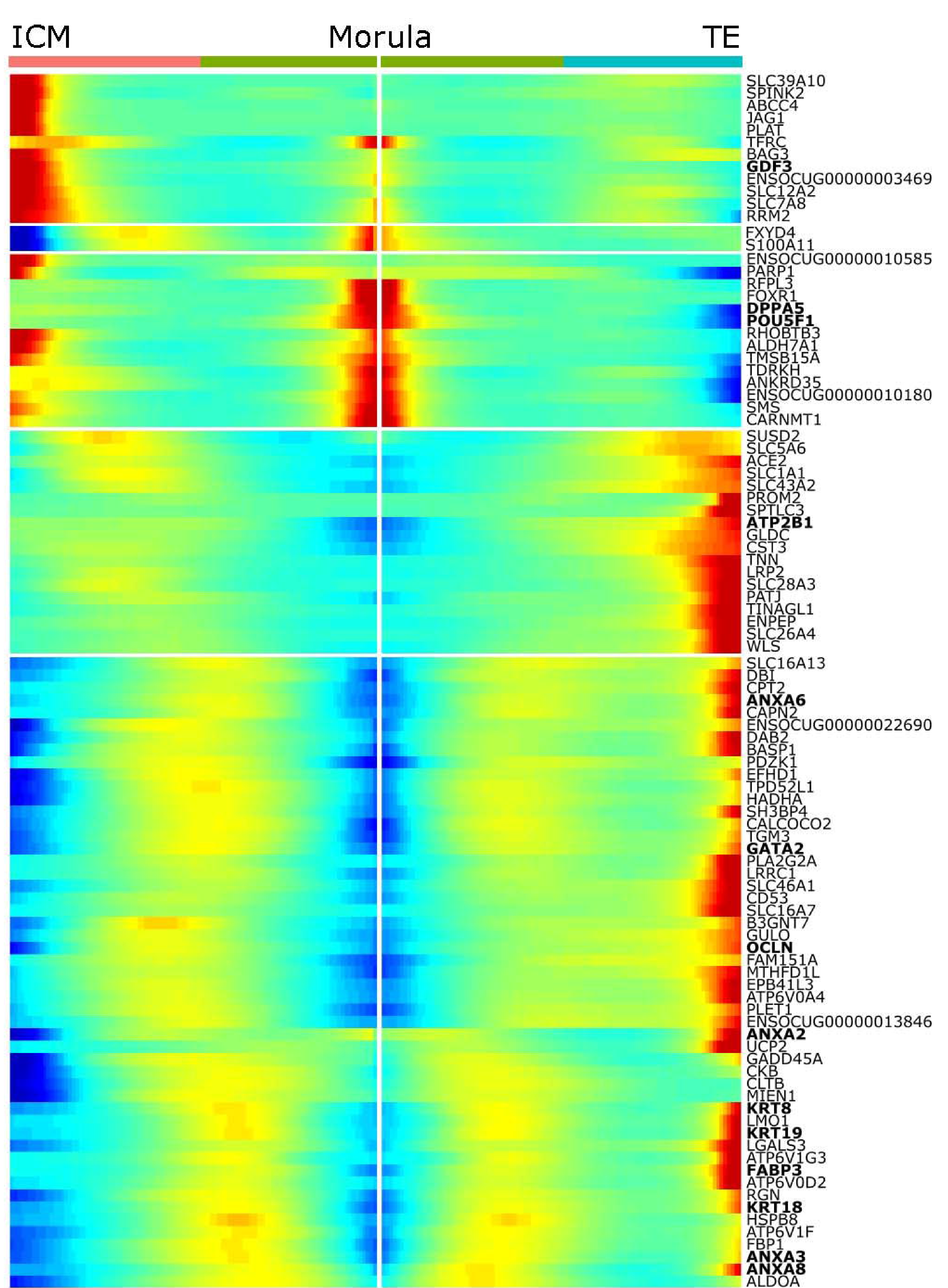

]

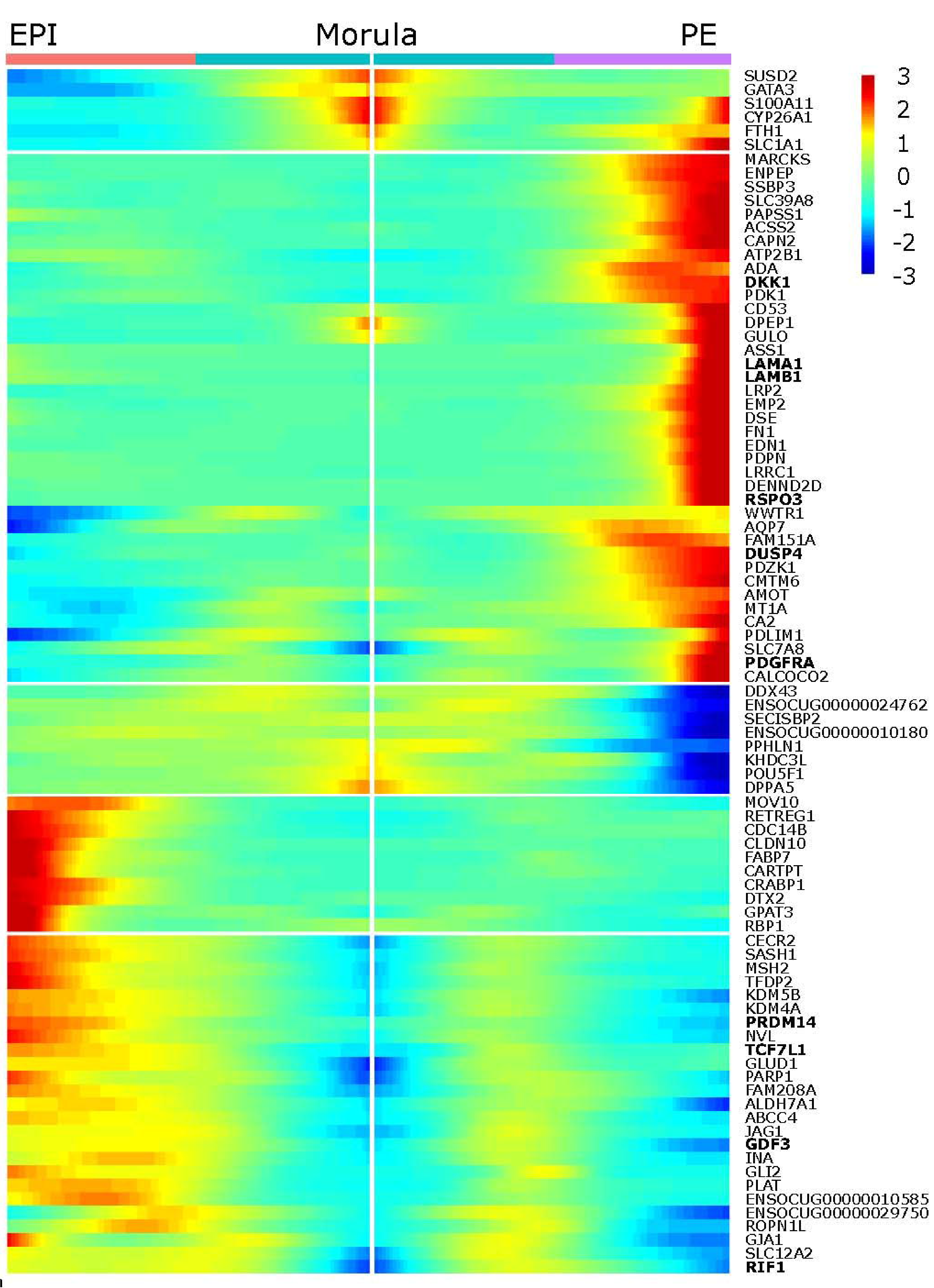


Figure 4: Transcriptome map of the pluripotency continuum.

A

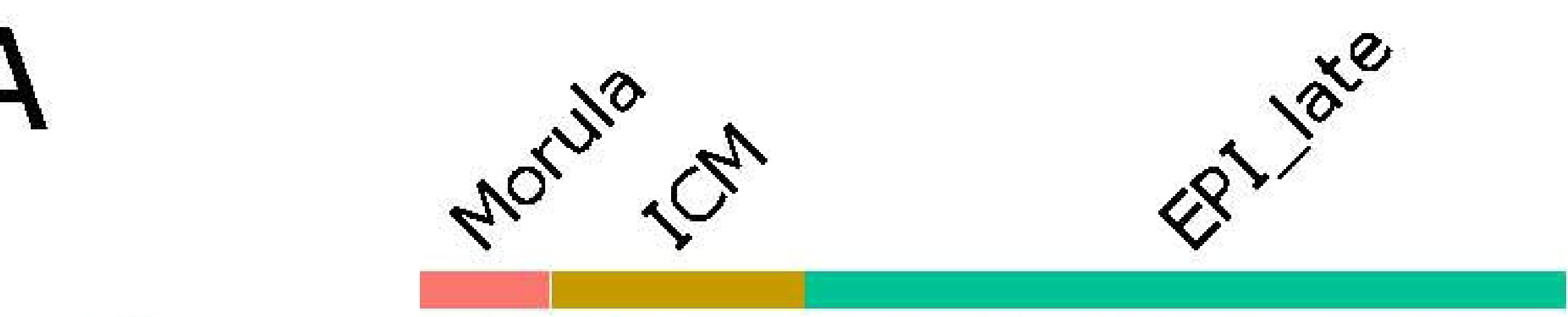

$\frac{0}{0}$

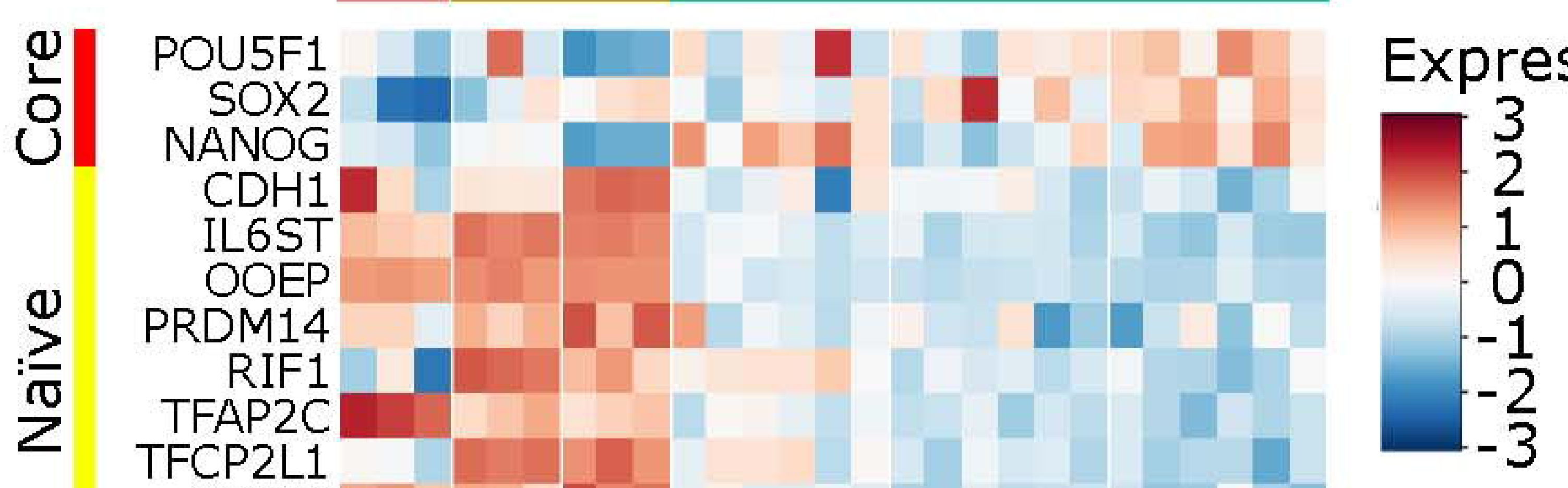

C
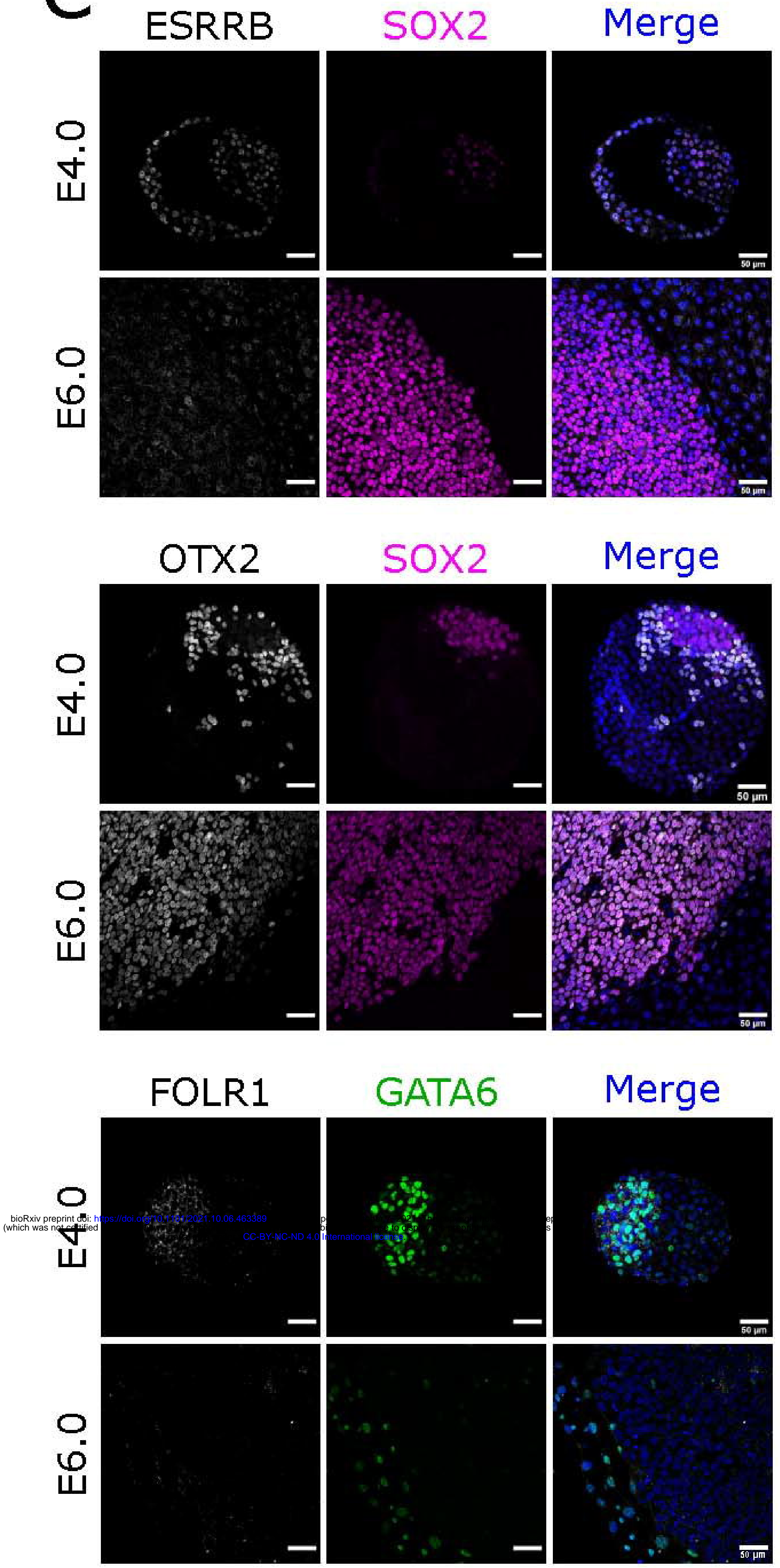

GATA6
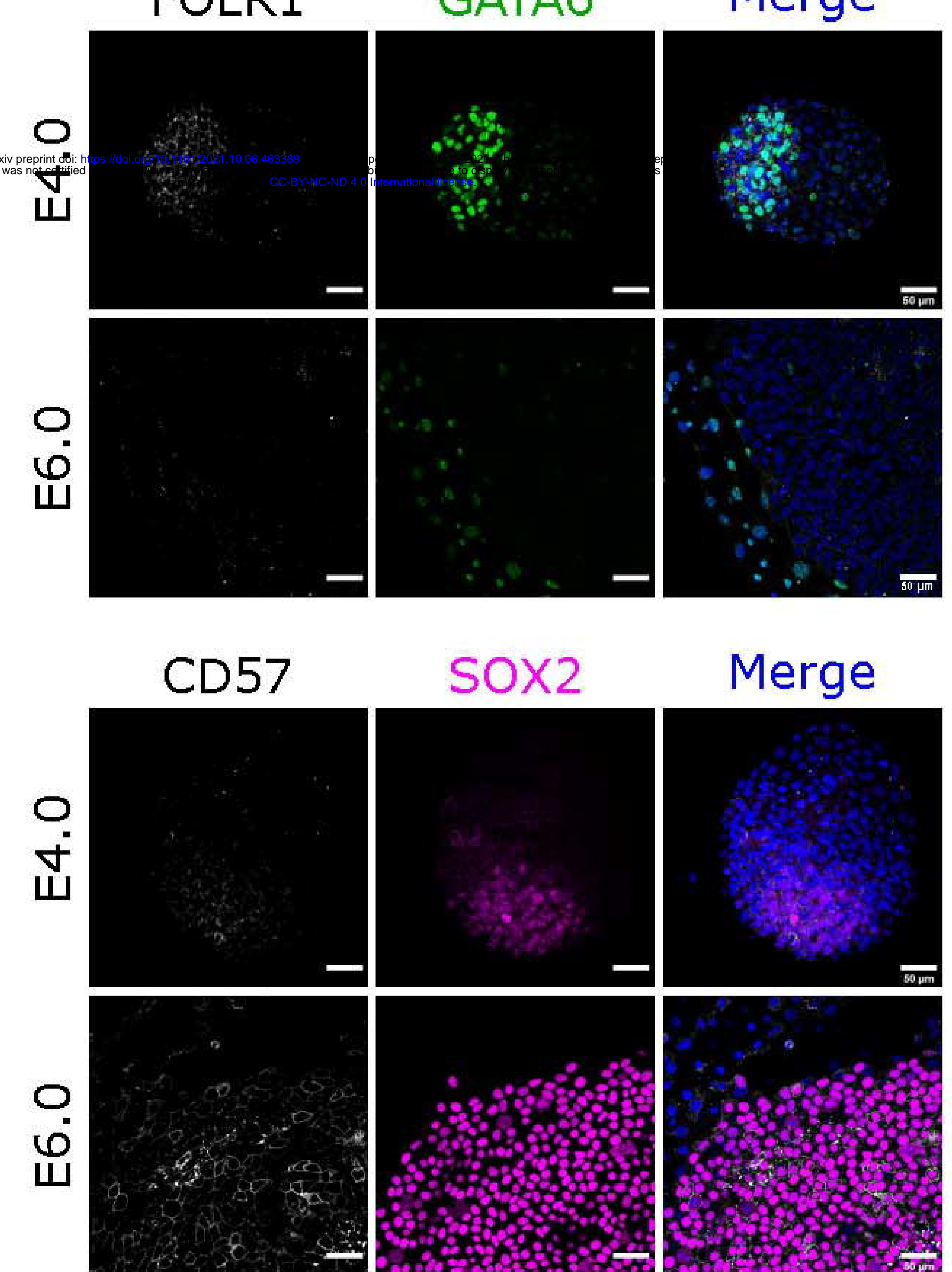

B

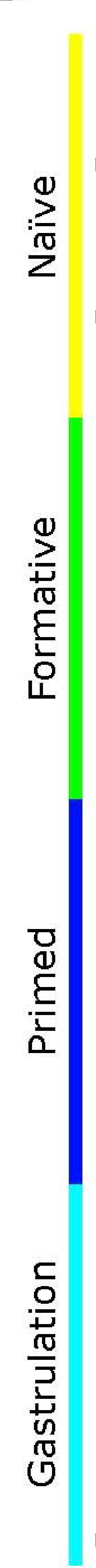

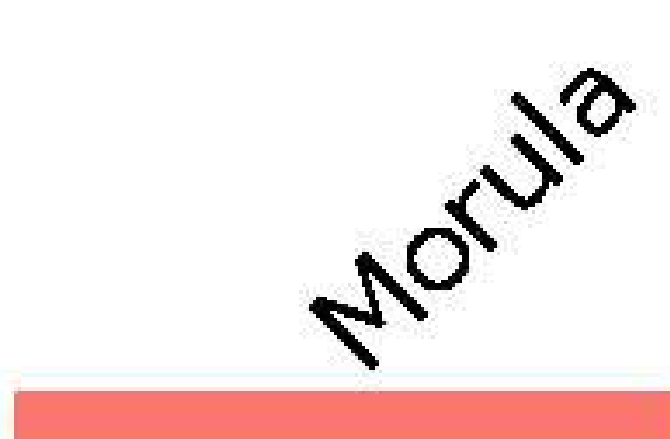

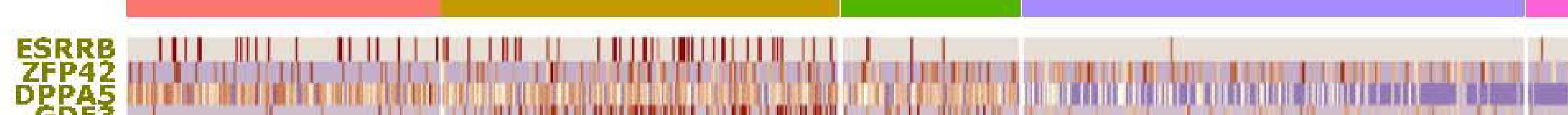
Expression

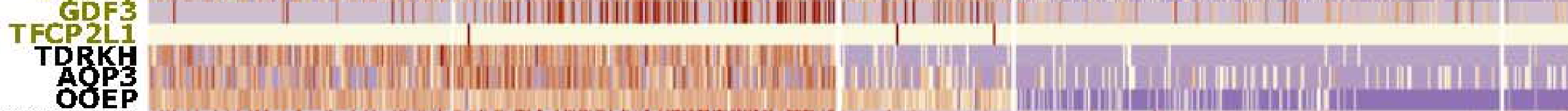

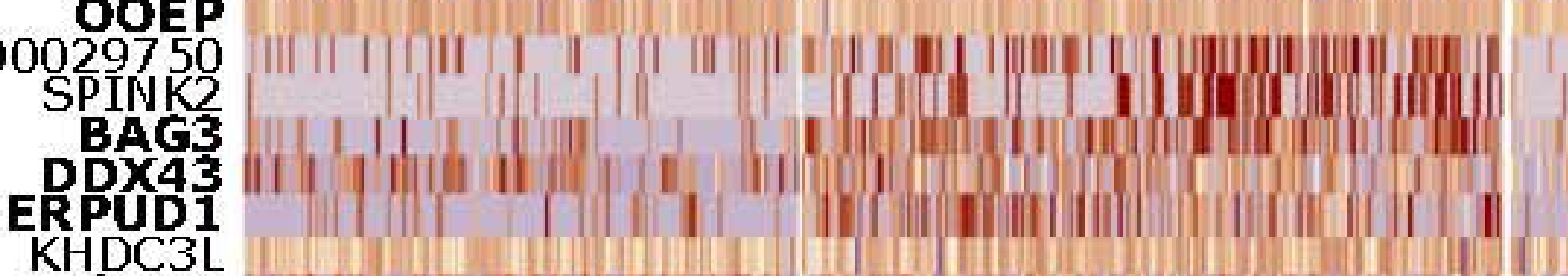

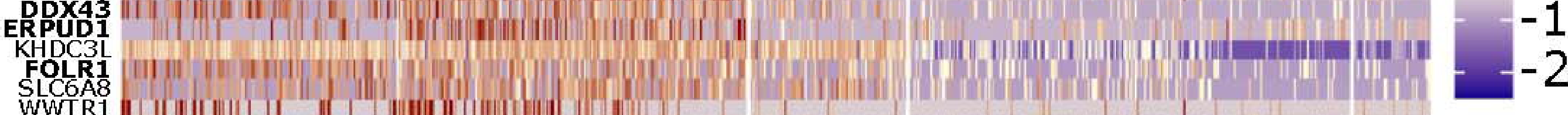
-

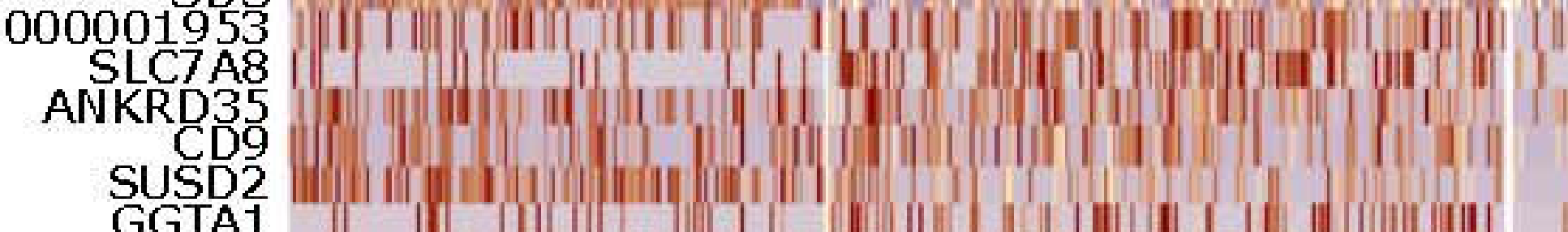

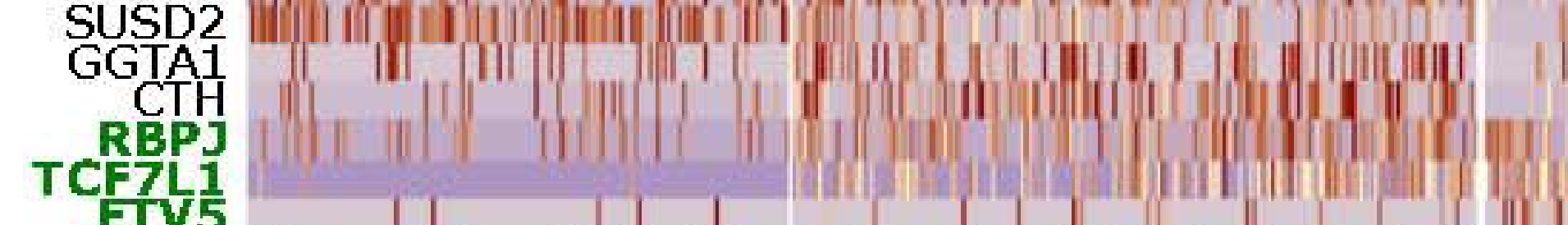

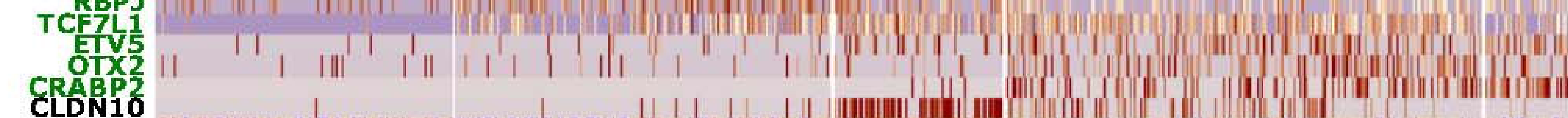

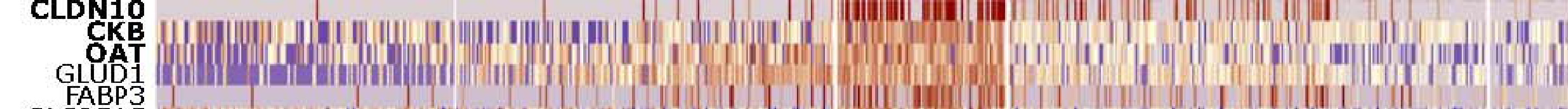
ALLCLA5

ANXA

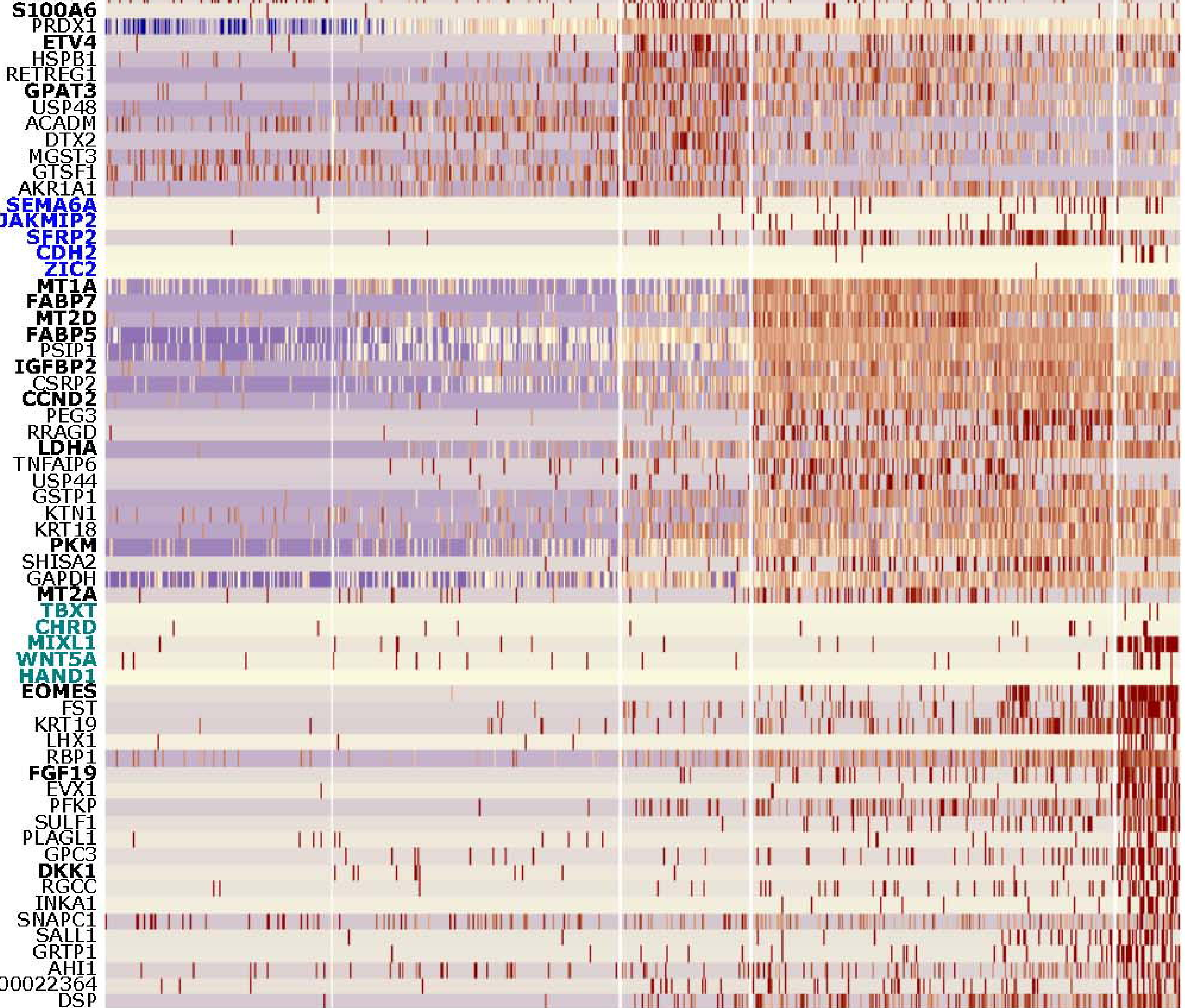

ENSOCUG00000022061
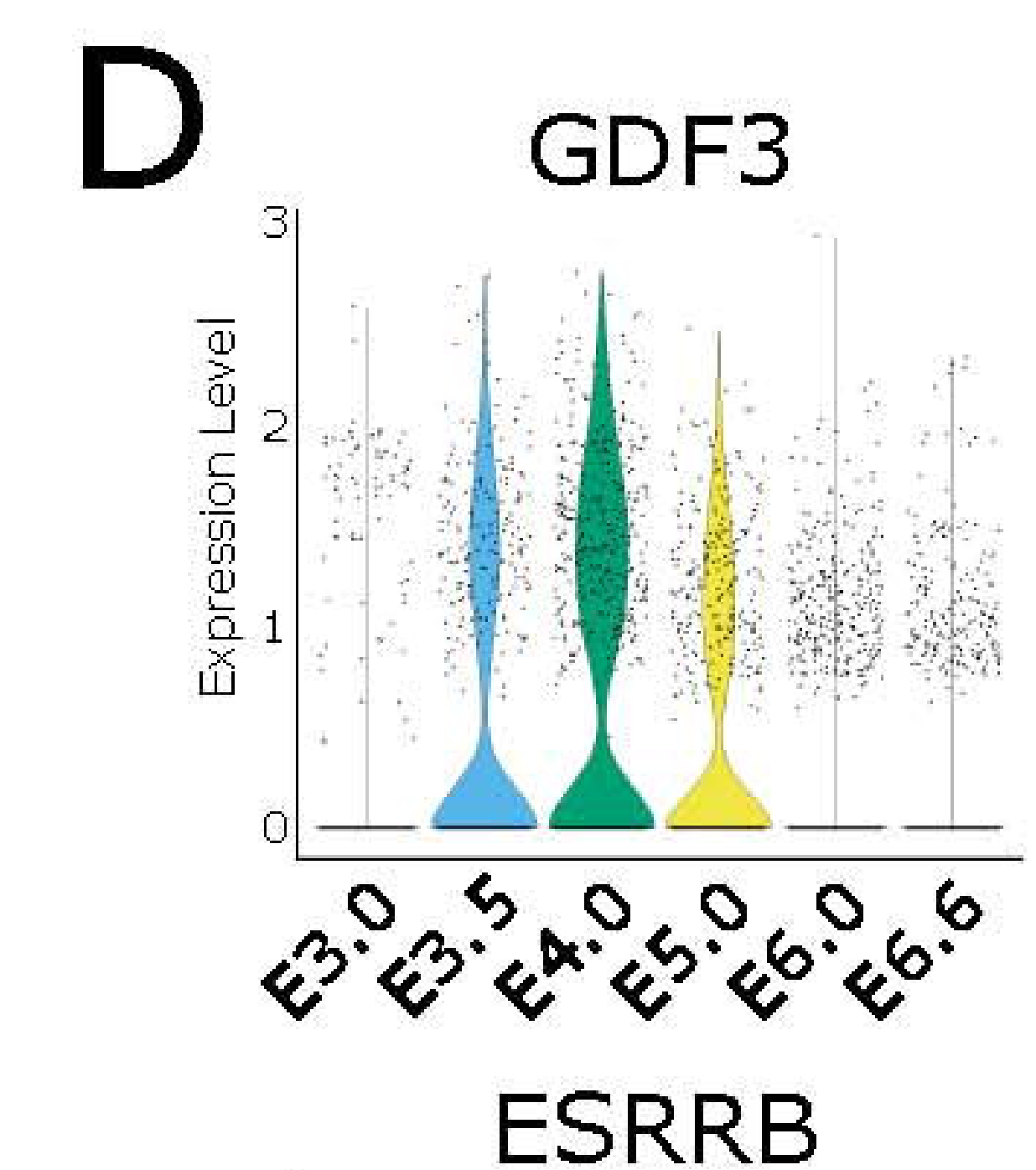

OTX2

SFRP2

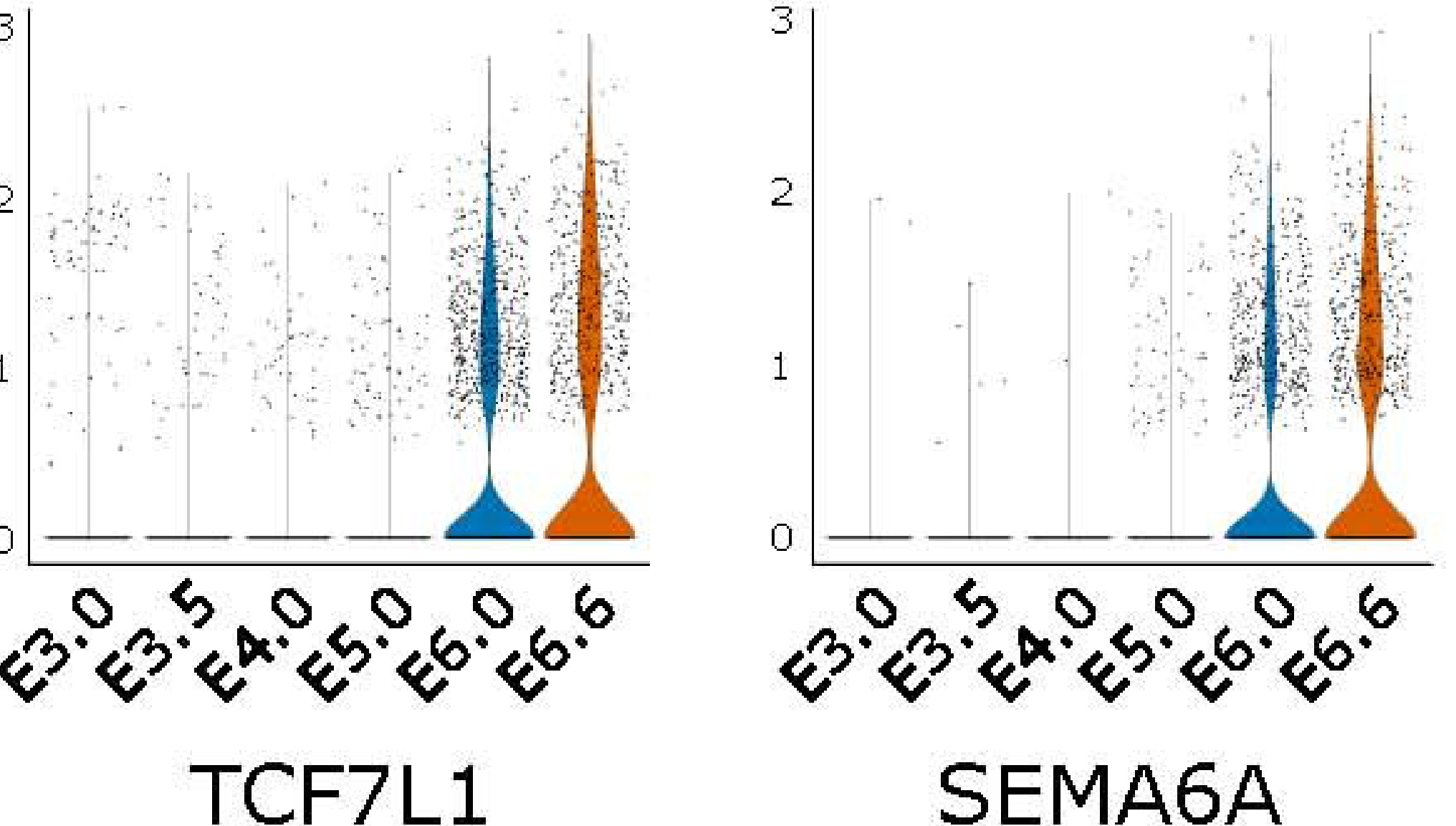

E
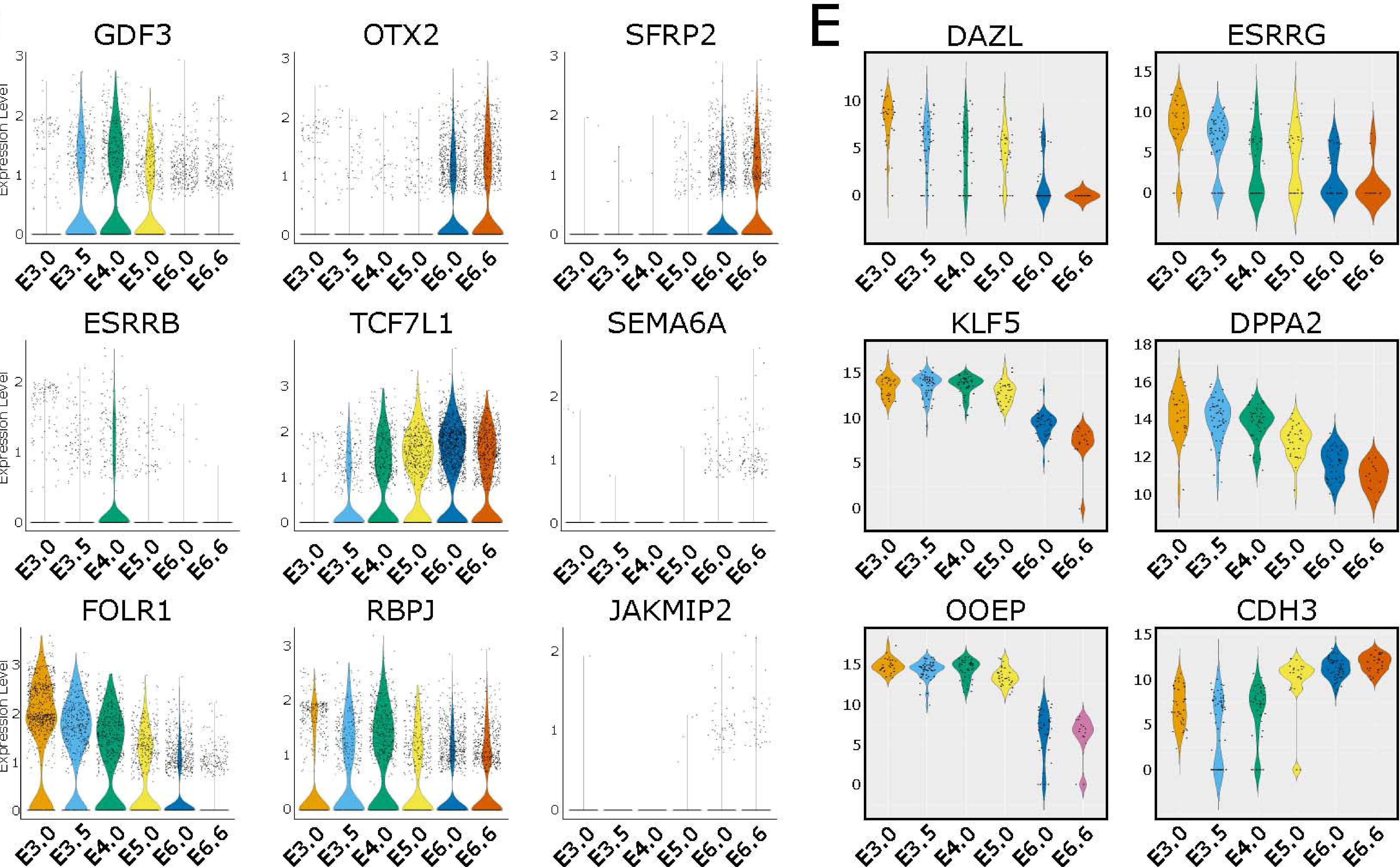

BAG3

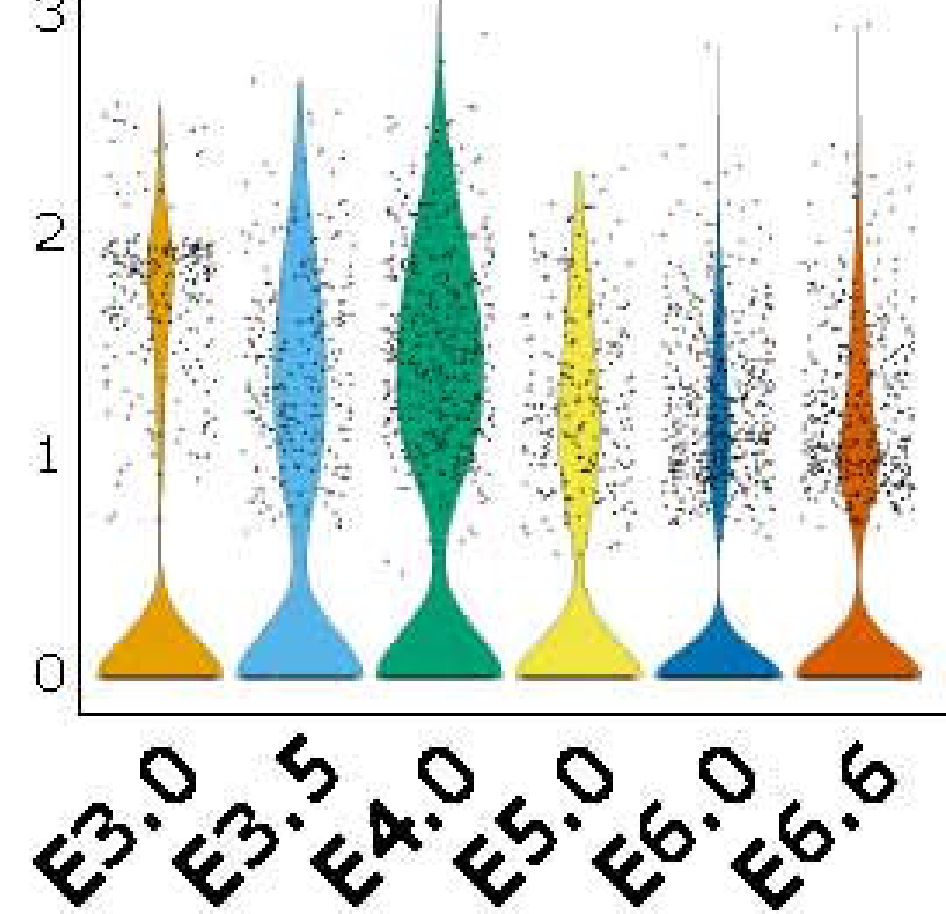

CLDN10

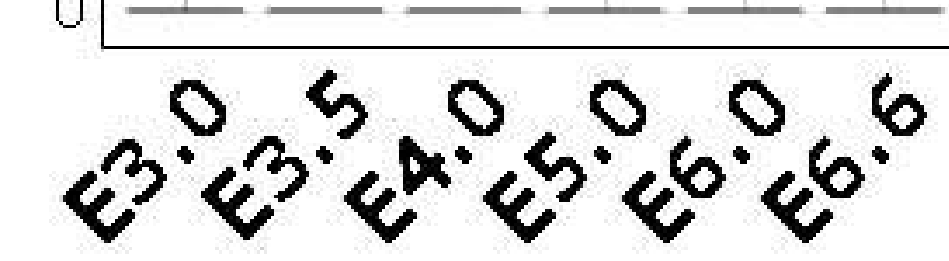

FABP7
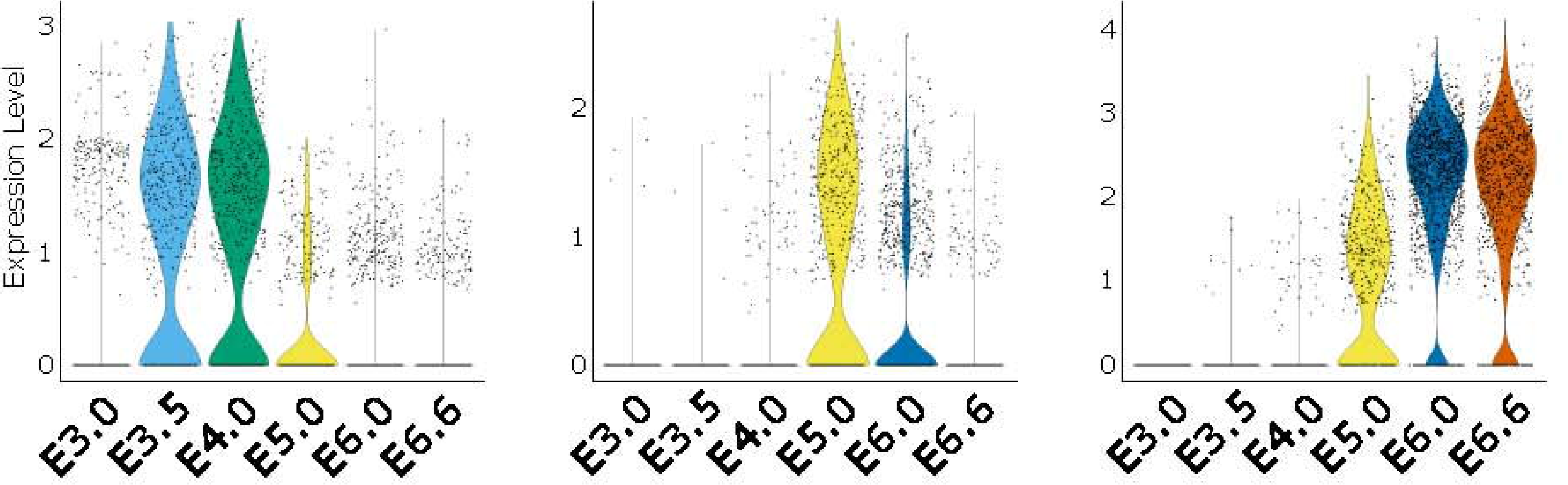

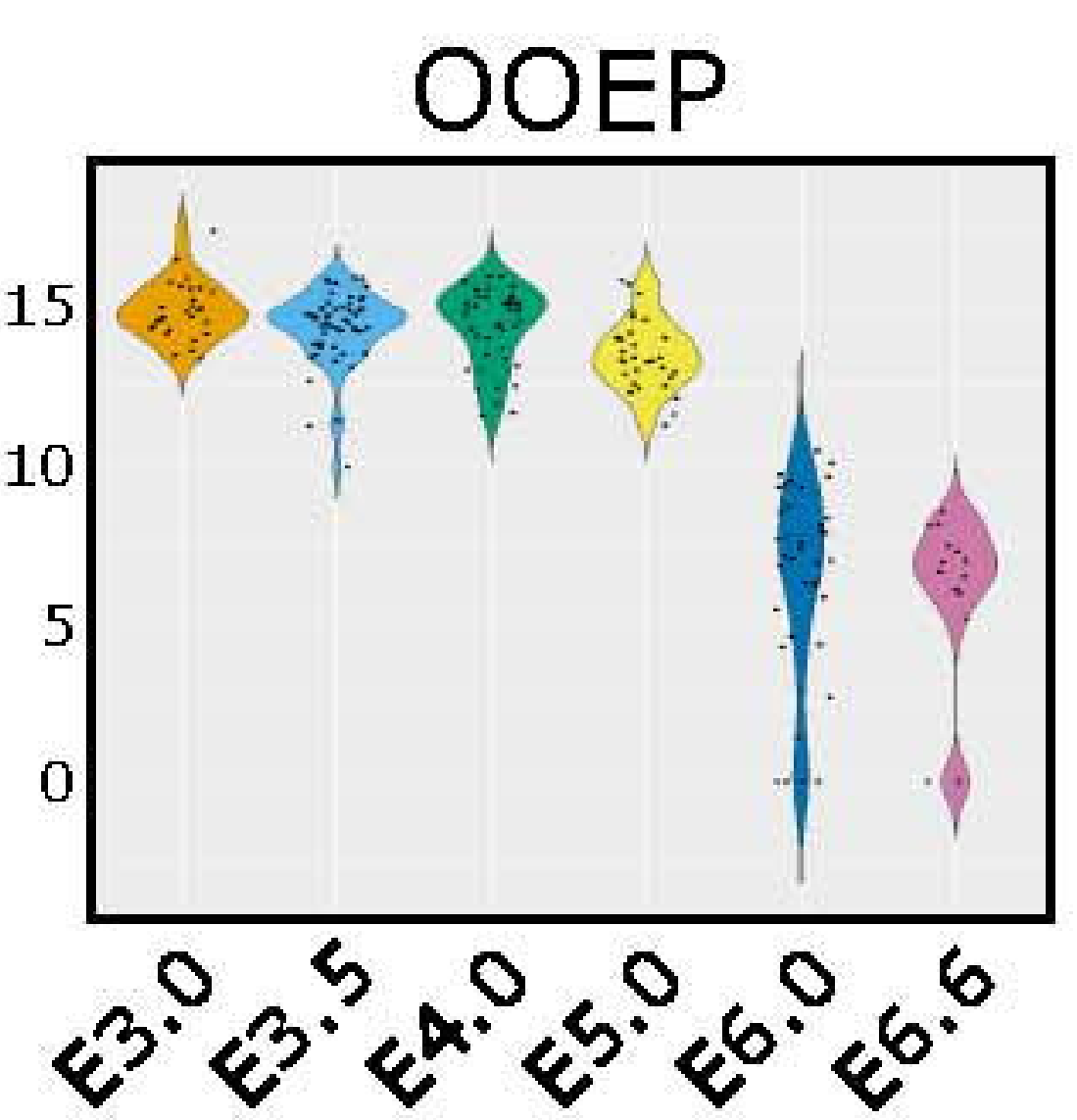

FBXO15

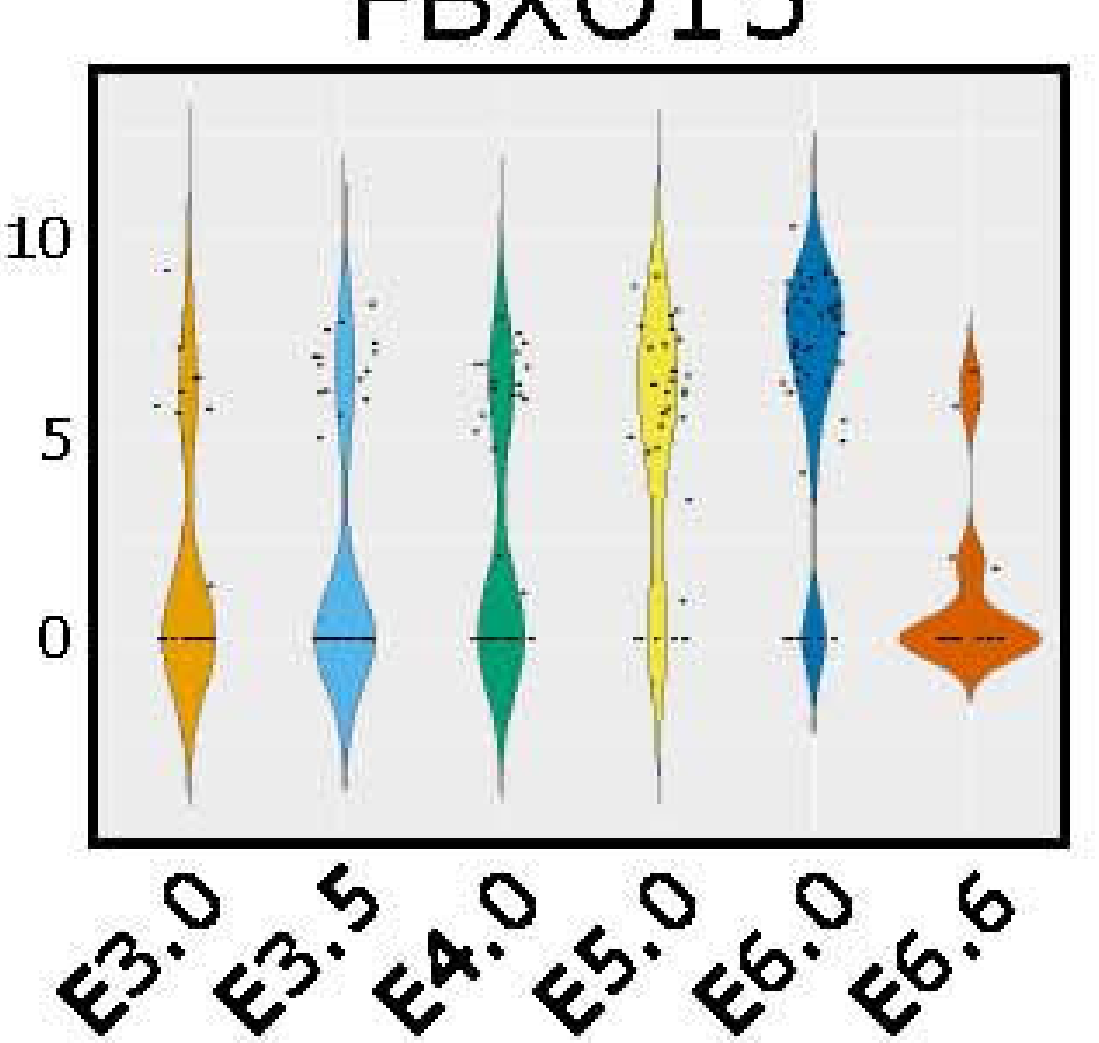

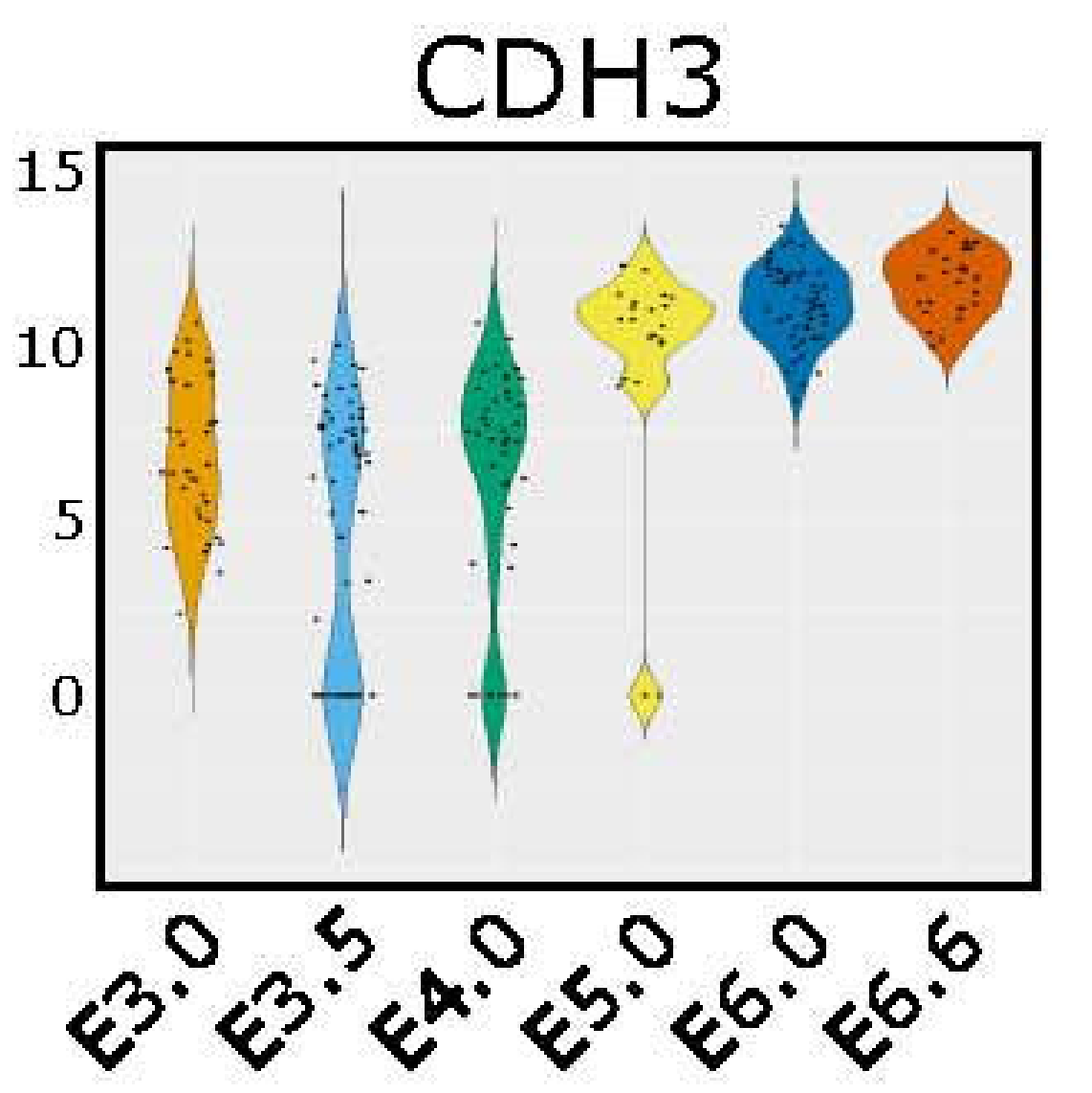

TDGF1

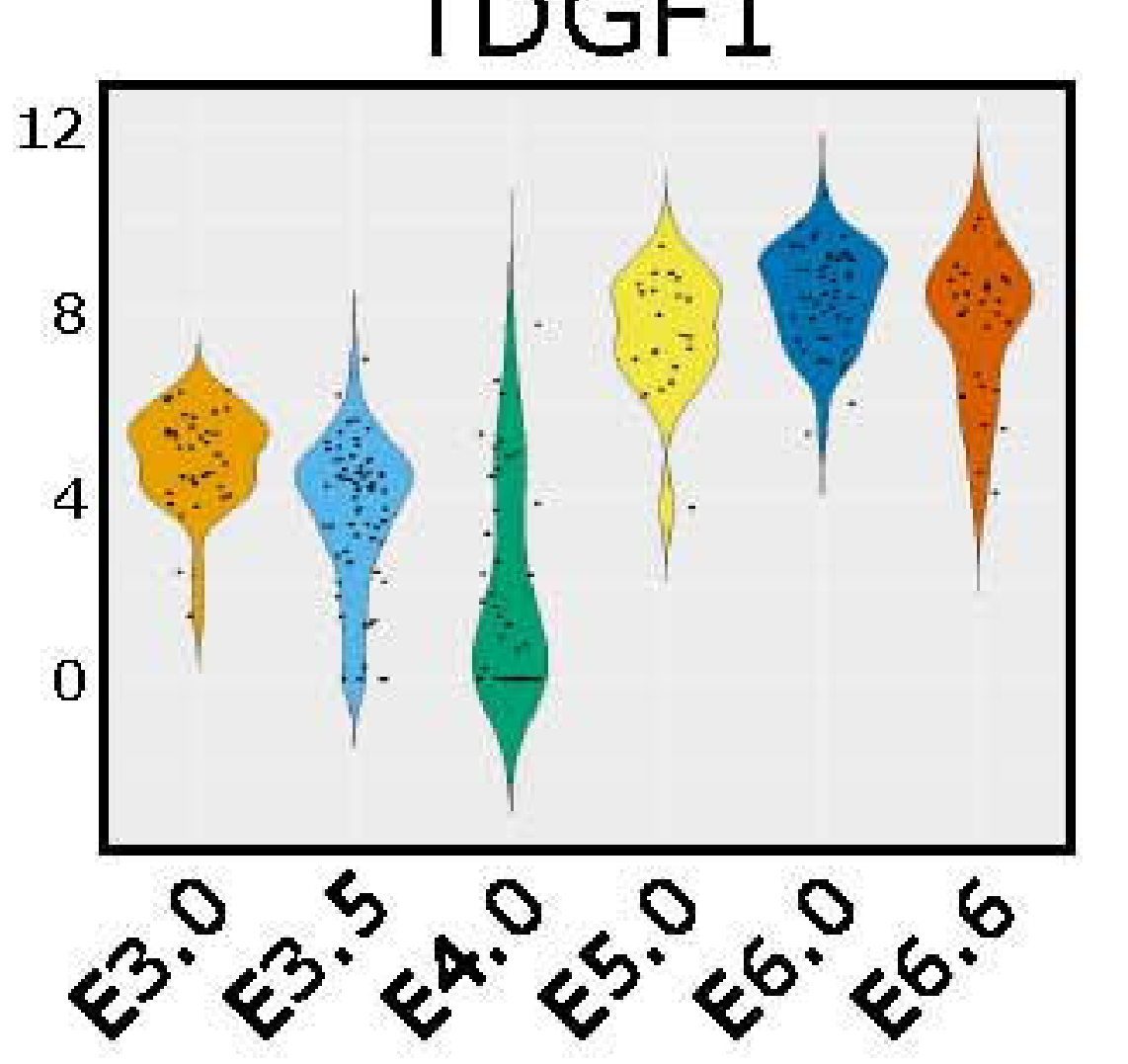


Figure 6: Metabolic characterization of the pluripotency continuum.

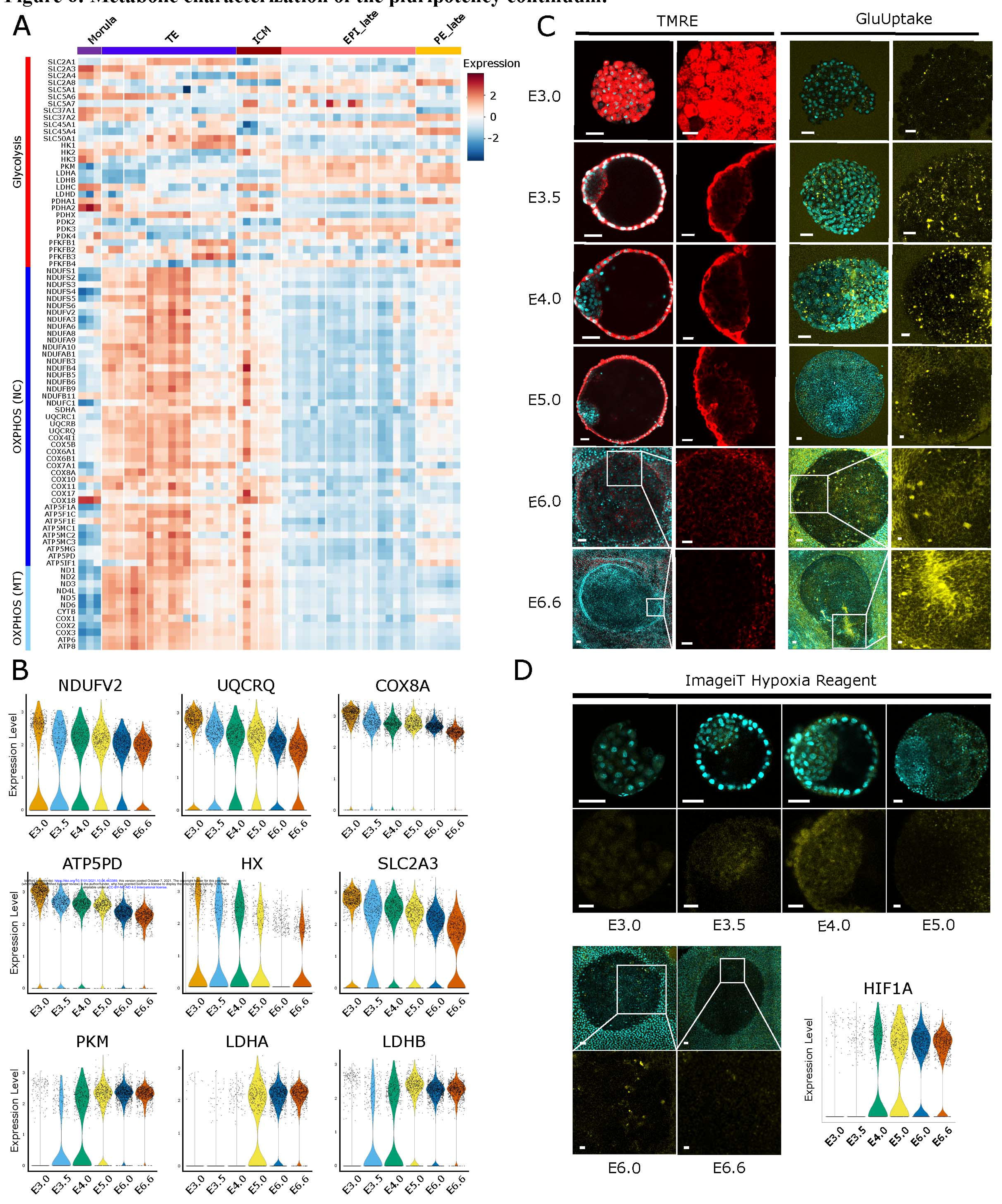


Figure 7: Defining markers of naïve pluripotency in rabbits.

A

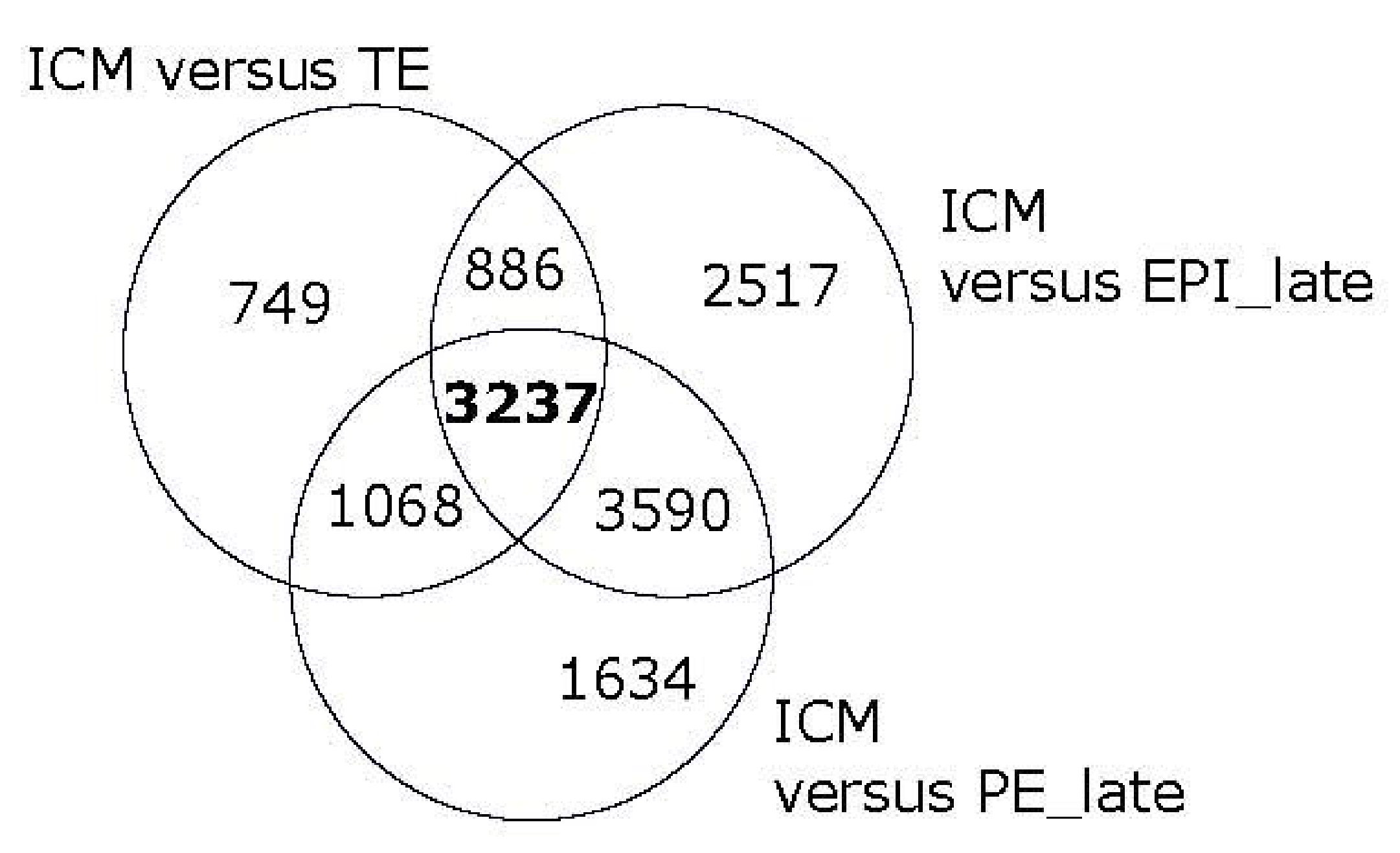

B

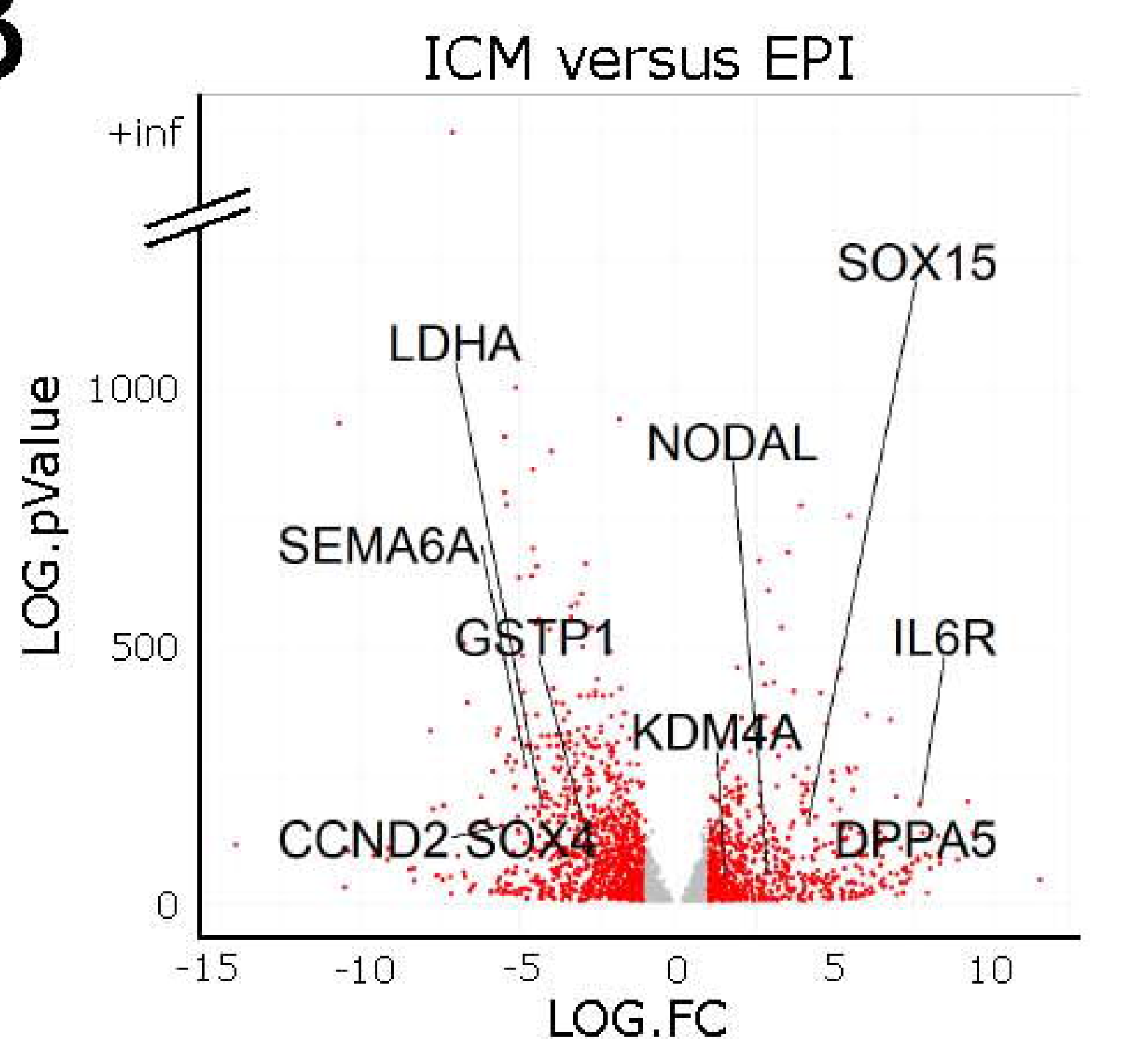

ICM versus $\mathrm{PE}$

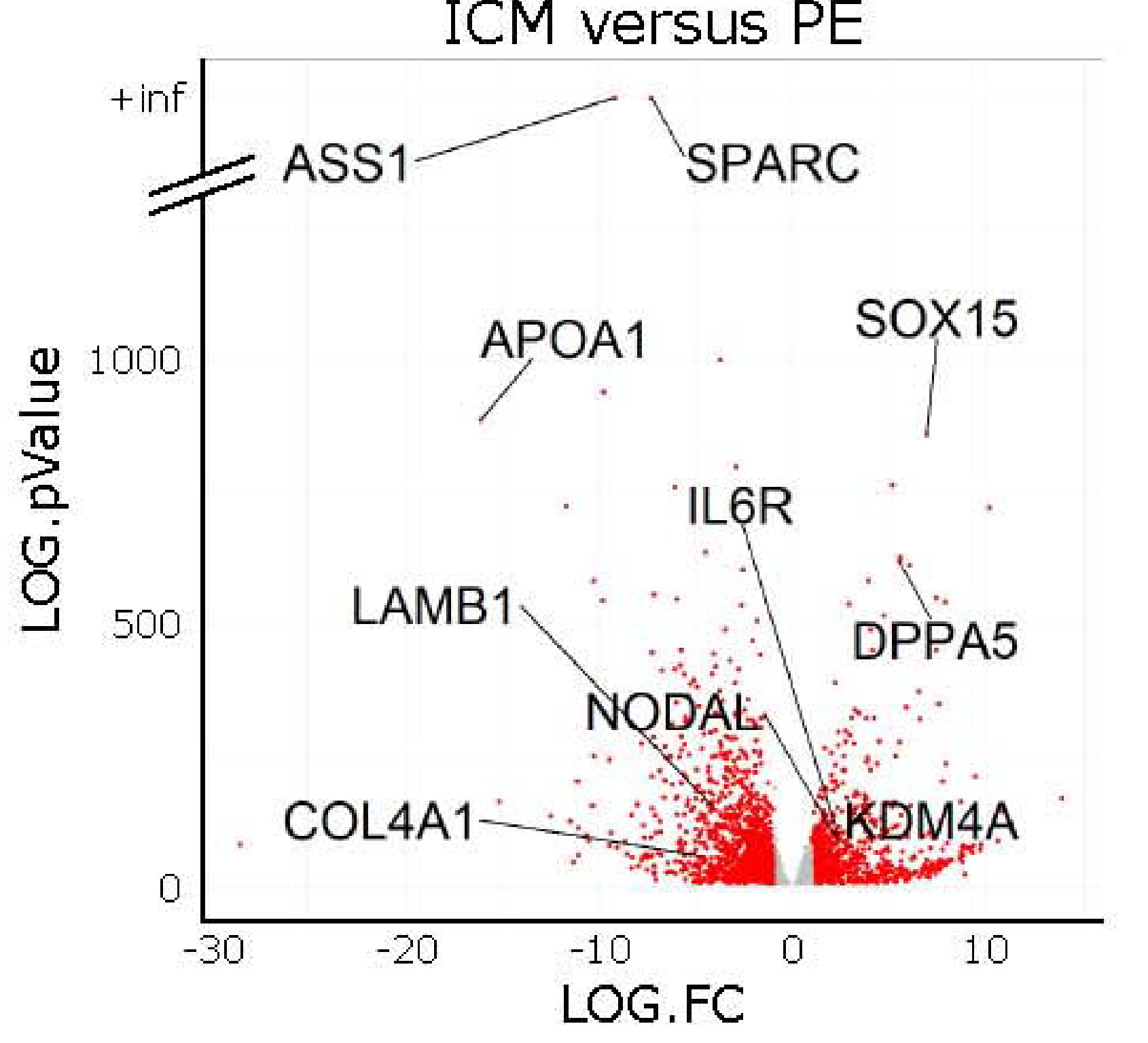

ICM versus TE

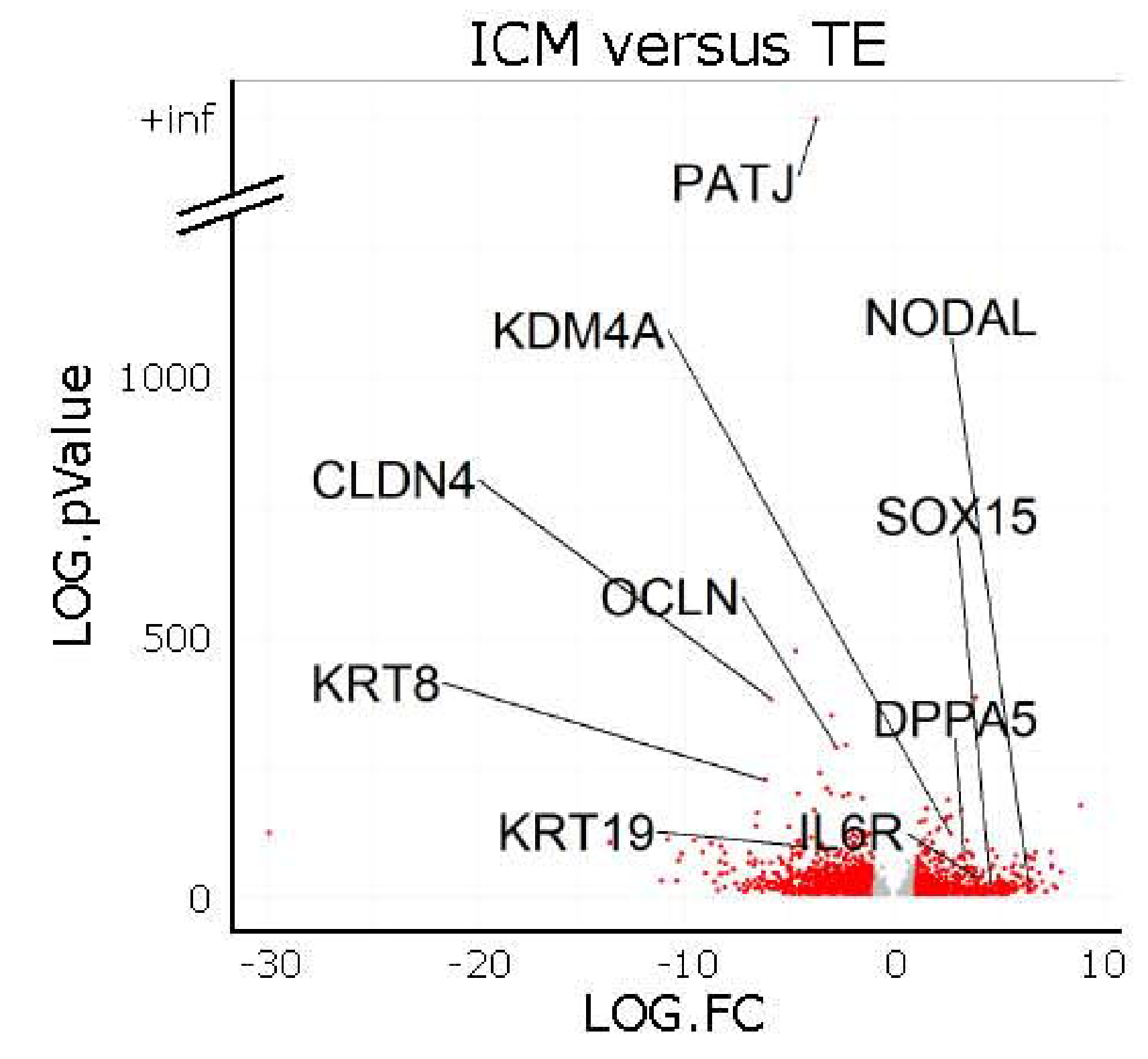

C

GO: Biological Process

$\operatorname{minus}_{0,5}$ Log $_{25}$ pValue

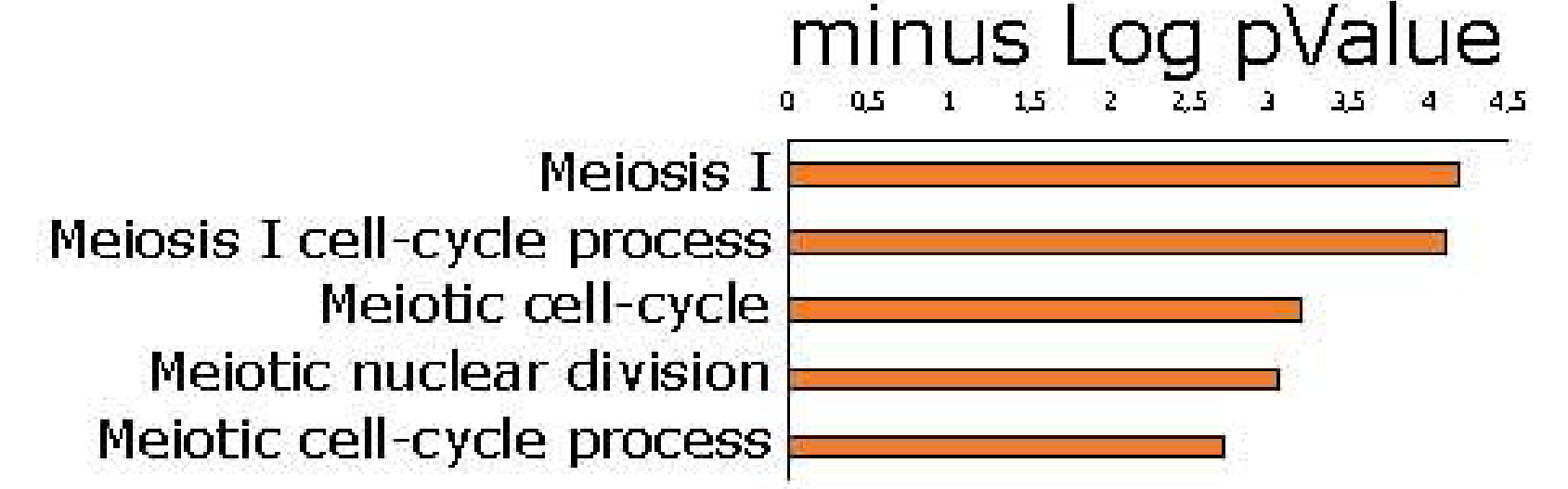

$\begin{array}{lllllllll}0 & 0,5 & 1 & 1,5 & 2 & 2,5 & 3 & 3,5 & 4\end{array}$

Obsolete oxidation-reduction process
Small molecule metabolic process
Organic acid metabolic process

Cell-substrate adhesion

D

\section{KEGG pathways}

$\operatorname{minus~Log~pValue~}_{0.5}$

Pathways in cancer
Signaling pathways regulating pluripotency

poripotency
oftem cells

ECM-receptor interaction

Vitamin digestion and absorption

Lysosome

G

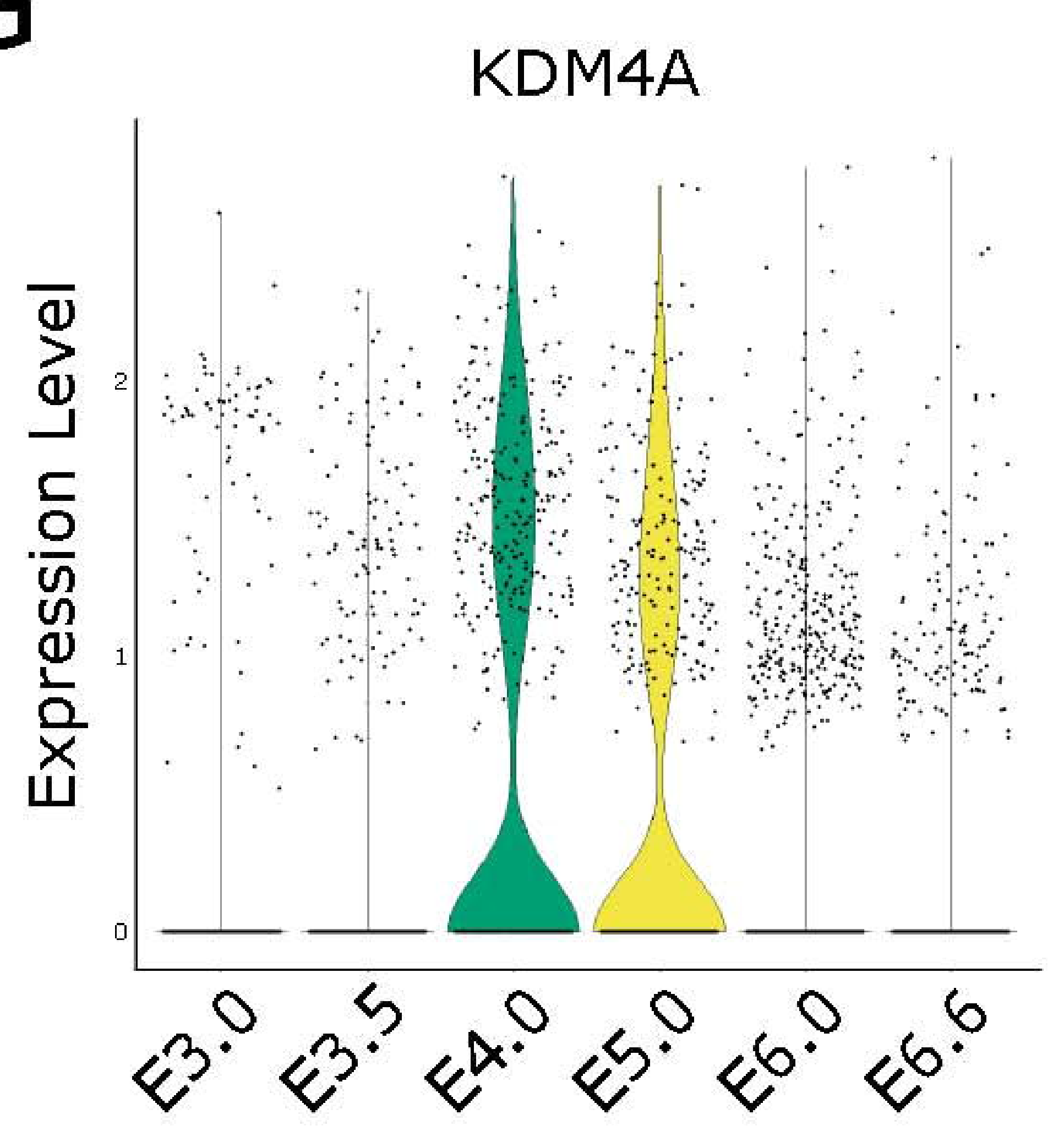

J
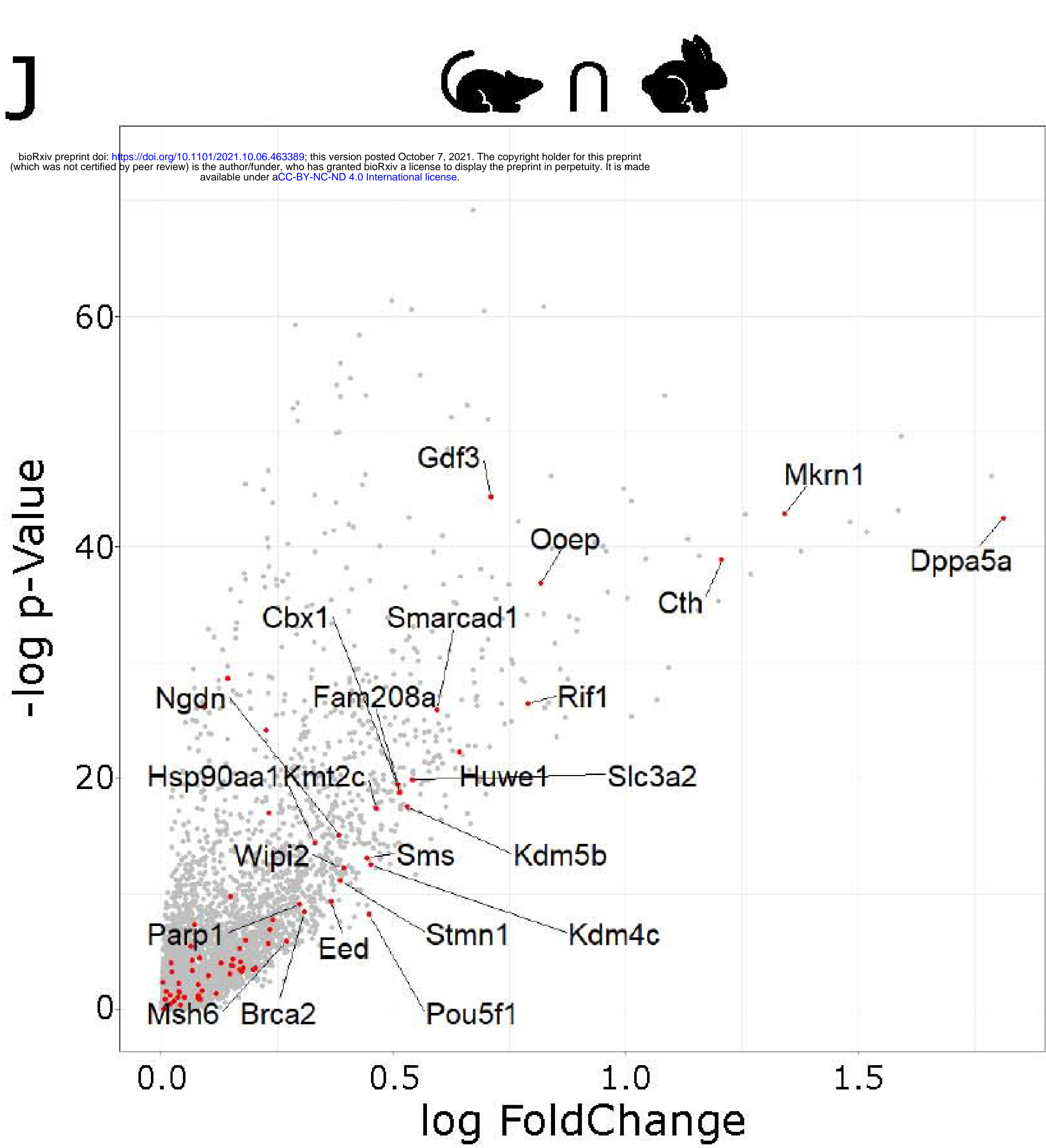

$\mathrm{H}$

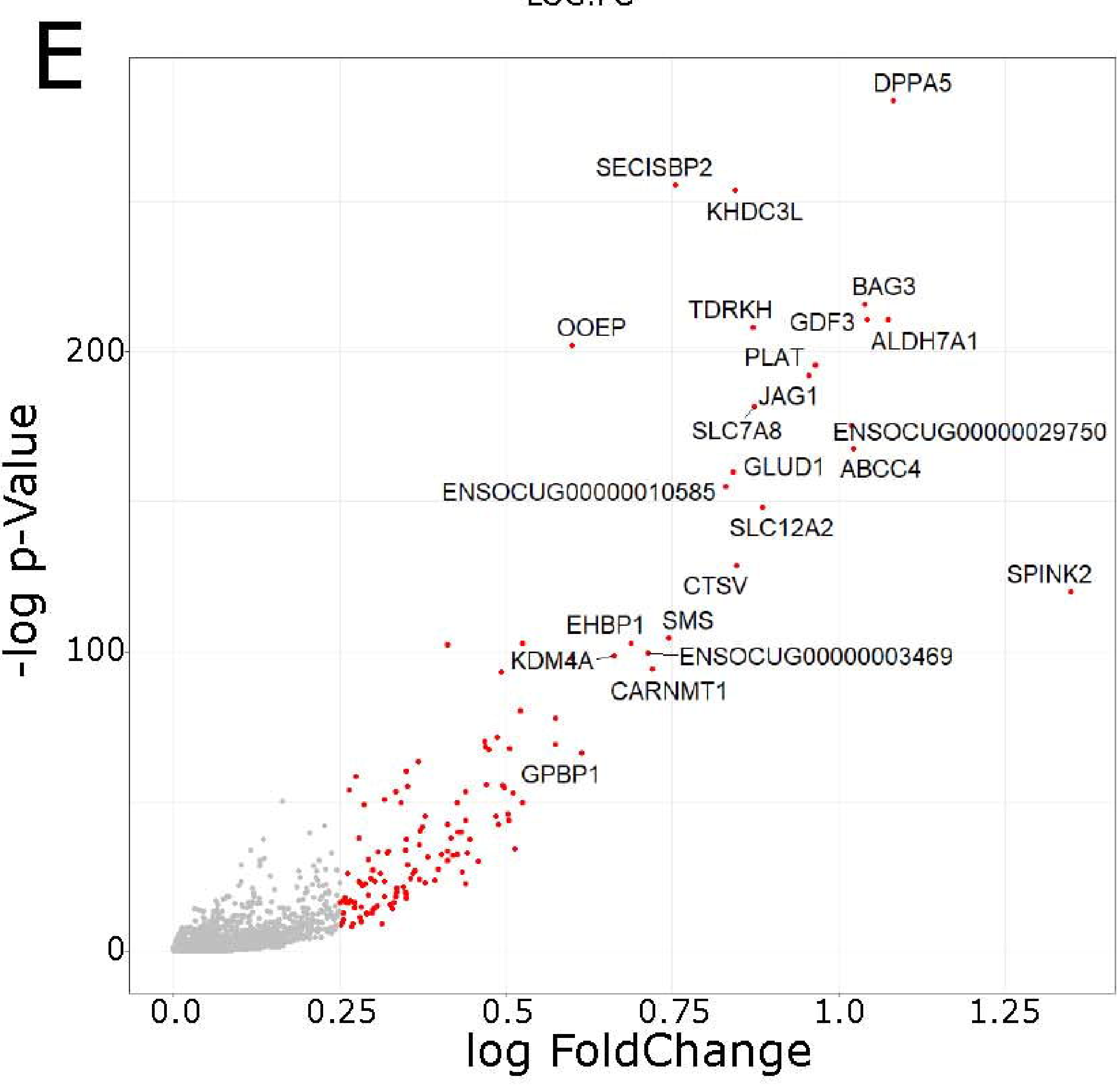

F

GO: Biological Process

${ }_{1} \operatorname{minus}_{2}$ Log pValue
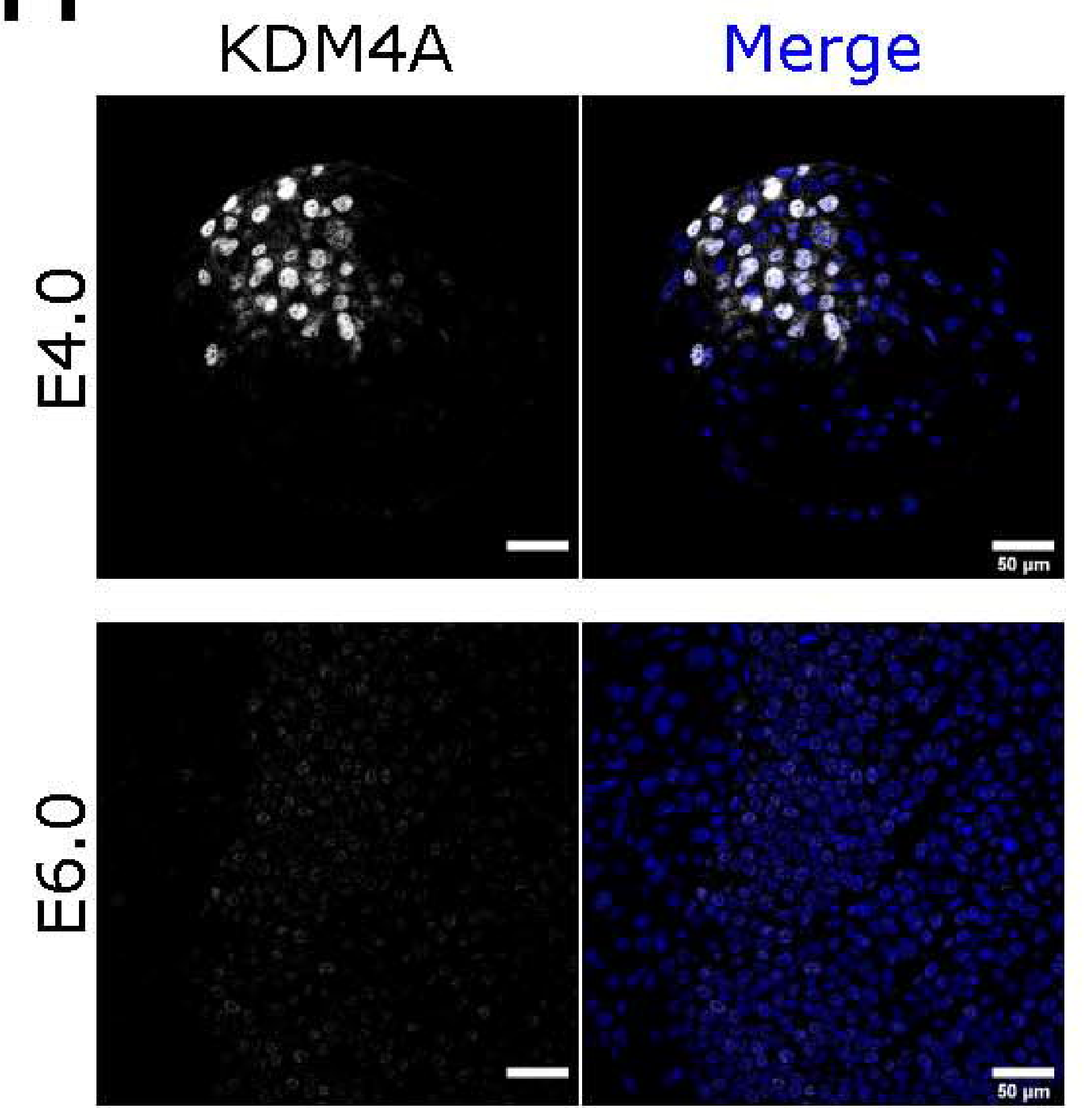

K
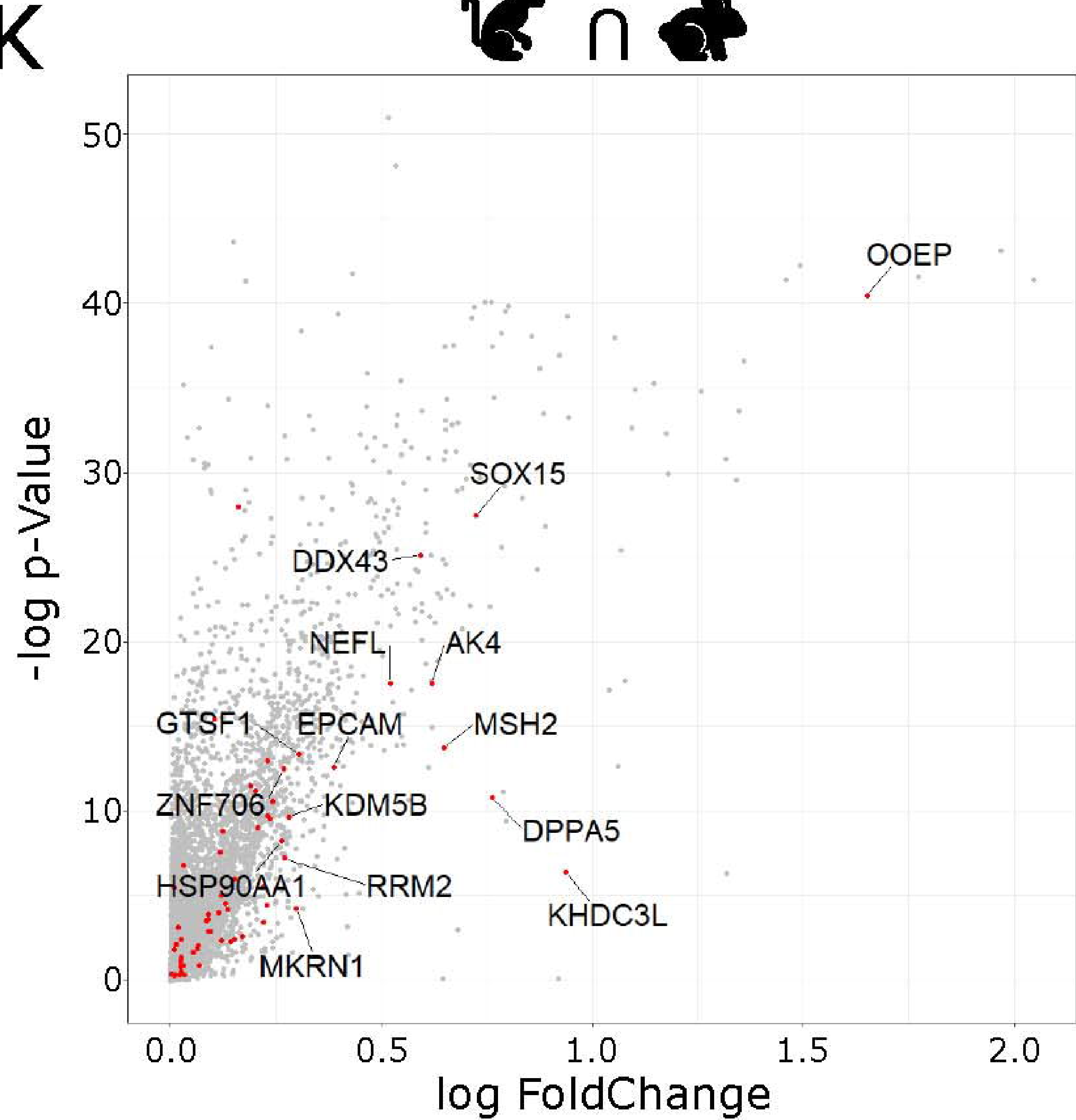

I Chromatin Organization Negative Regulation of Nucleobase-Containing Compound Histone Modification DNA Repair
Degative Regulation of RNA Metabolic Process Negative Regulation of Cellular Biosynthetic Process Negative Regulation of Biosynthetic Process Negative Regulation of DNA Metabolic Process Negative Regulation of Cellular Macromolecule Biosynthetic Process Negative Regulation of Macromolecule Biosynthetic Process DNA Metabolic Process Mismatch Repair

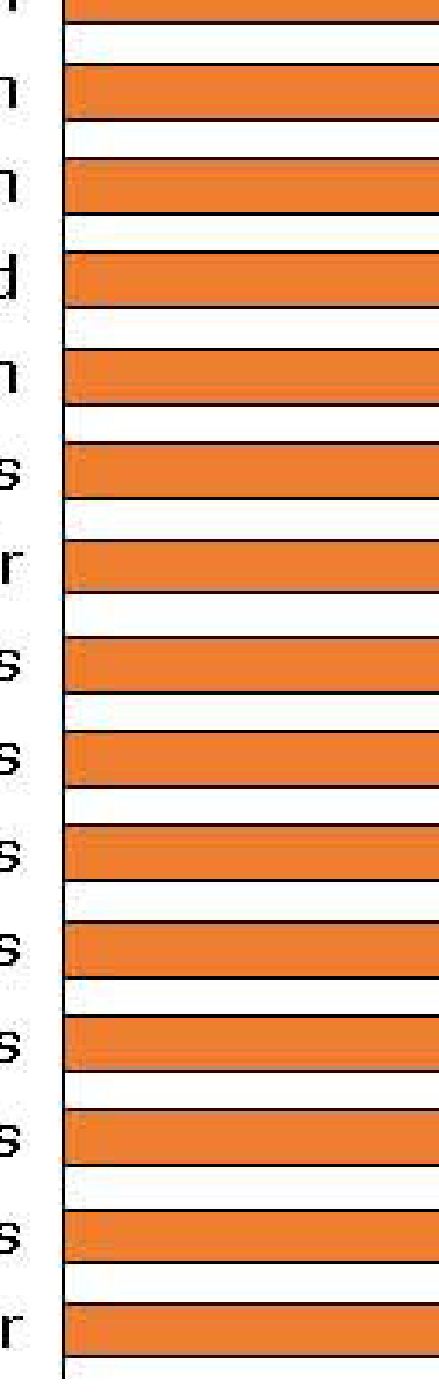

$\underset{5}{p \text { Value }}$
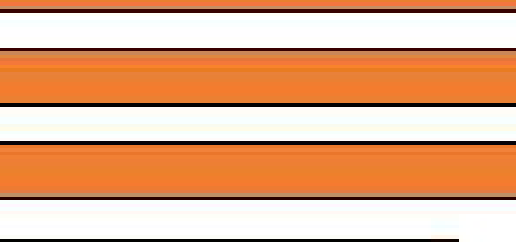

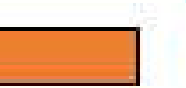

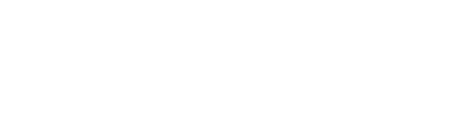

